\title{
Theory of coherent phonons in carbon nanotubes and graphene nanoribbons
}

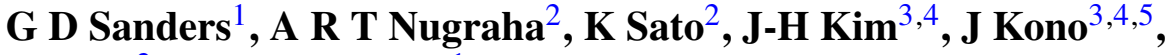 \\ R Saito ${ }^{2}$ and C J Stanton ${ }^{1}$ \\ ${ }^{1}$ Department of Physics, University of Florida, Box 118440, Gainesville, FL 32611-8440, USA \\ 2 Department of Physics, Tohoku University, Sendai 980-8578, Japan \\ ${ }^{3}$ Department of Electrical and Computer Engineering, Rice University, Houston, TX 77005, USA \\ 4 The Richard E Smalley Institute for Nanoscale Science and Technology, Rice University, Houston, \\ TX 77005, USA \\ ${ }^{5}$ Department of Physics and Astronomy, Rice University, Houston, TX 77005, USA \\ E-mail: sanders@phys.ufl.edu and nugraha@flex.phys.tohoku.ac.jp
}

Received 26 October 2012, in final form 6 February 2013

Published 11 March 2013

Online at stacks.iop.org/JPhysCM/25/144201

\begin{abstract}
We survey our recent theoretical studies on the generation and detection of coherent radial breathing mode (RBM) phonons in single-walled carbon nanotubes and coherent radial breathing like mode (RBLM) phonons in graphene nanoribbons. We present a microscopic theory for the electronic states, phonon modes, optical matrix elements and electron-phonon interaction matrix elements that allows us to calculate the coherent phonon spectrum. An extended tight-binding (ETB) model has been used for the electronic structure and a valence force field (VFF) model has been used for the phonon modes. The coherent phonon amplitudes satisfy a driven oscillator equation with the driving term depending on the photoexcited carrier density. We discuss the dependence of the coherent phonon spectrum on the nanotube chirality and type, and also on the graphene nanoribbon mod number and class (armchair versus zigzag). We compare these results with a simpler effective mass theory where reasonable agreement with the main features of the coherent phonon spectrum is found. In particular, the effective mass theory helps us to understand the initial phase of the coherent phonon oscillations for a given nanotube chirality and type. We compare these results to two different experiments for nanotubes: (i) micelle suspended tubes and (ii) aligned nanotube films. In the case of graphene nanoribbons, there are no experimental observations to date. We also discuss, based on the evaluation of the electron-phonon interaction matrix elements, the initial phase of the coherent phonon amplitude and its dependence on the chirality and type. Finally, we discuss previously unpublished results for coherent phonon amplitudes in zigzag nanoribbons obtained using an effective mass theory.
\end{abstract}

(Some figures may appear in colour only in the online journal)

\section{Contents}

1. Introduction

2. Coherent phonons

3. Theory for generation and detection of coherent phonons
3.1. Electronic states

3.2. Optical properties

3.3. Phonon modes

4 3.4. Electron-phonon matrix elements 10

3.5. Generation of coherent phonons 13

6

3.6. Detection of coherent phonons 
4. Coherent phonons in carbon nanotubes

4.1. Initial phase of the RBM CP amplitude

4.2. CP in micelle suspended SWNTs

4.3. Polarization dependence of the $\mathrm{CP}$ signal in aligned nanotube films

5. Coherent phonons in graphene nanoribbons

5.1. Armchair nanoribbons

5.2. Zigzag nanoribbons

6. Summary and conclusions

Acknowledgments

References

\section{Introduction}

For over twenty five years, scientists have been keenly interested in studying carbon based nanostructures because of their unique optical and electronic properties and because they provide ideal systems for studying low-dimensional electron systems, such as 0D buckyballs [1-3], 2D graphene [4-6], and 1D carbon nanotubes and graphene nanoribbons [7, 8]. The unique properties of these systems hold promise for a wide range of future applications from drug delivery systems to $\mathrm{THz}$ detectors and lasers to high speed transistors [9-11]. In this paper, we present an overview of our theoretical studies of one specific dynamical optical property, namely coherent phonon (CP) spectroscopy in two distinct but related classes of carbon nanostructures: single-walled carbon nanotubes (SWNTs) and graphene nanoribbons (GNRs).

Coherent phonon spectroscopy uses ultrafast pumpprobe spectroscopy in which either transient differential transmission $(\Delta T / T)$ or differential reflection $(\Delta R / R)$ oscillations vibrating at phonon frequencies are measured by the probe pulse as a function of delay time relative to an ultrafast pump pulse. Taking the Fourier transform of the transient transmission or reflection signal after subtracting a slowly varying background produces the coherent phonon spectrum. In order to observe macroscopic properties like $\Delta T / T$ or $\Delta R / R$ in a solid vibrating at phonon frequencies, it is necessary for the phonon modes in question to vibrate in phase. Because these modes vibrate in phase, they are referred to as coherent phonons. Coherent phonon oscillations in a solid are excited by electrons and holes generated by an ultrafast pump which then interact with phonons through the electron-phonon interaction. For coherent phonons to be excited, it is necessary for the pump pulse duration to be short in comparison with the phonon oscillation period so that all phonon oscillations start at the same time with the same phase. For example, to generate coherent radial breathing mode (RBM) phonons in a SWNT where the SWNT diameter vibrates at a typical frequency of $5 \mathrm{THz}$, the pump pulse duration should be shorter than $100 \mathrm{fs}$, while for coherent optic G band phonons in SWNTs and graphene with typical frequencies of $47 \mathrm{THz}$, the pump pulse duration needs to be as small as $10 \mathrm{fs}$.

Coherent phonons are widely observed in coherent phonon spectroscopy on a variety of materials [12-24]. The Fourier transform of the transient $\Delta T / T$ or $\Delta R / R$
6 due to coherent phonon oscillations produces a spectrum similar to Raman spectra from which information on phonons in solids can be obtained. In contrast with Raman spectroscopy, coherent phonon spectroscopy is free from Rayleigh scattering effects since we measure the transient $\Delta T / T$ or $\Delta R / R$ using the delayed probe beam and not from the scattered light. Thus the observed CP spectra is especially useful for measuring low frequency phonons with wavenumbers smaller than $100 \mathrm{~cm}^{-1}$ and for measuring spectra obtained by changing the laser excitation energy. In CP spectroscopy, the coherent phonon amplitude is large when (1) a strong optical absorption (or large joint density of states) occurs, (2) large electron-phonon matrix elements exist, (3) the pump pulse duration is sufficiently short compared with the phonon period and (4) the stimulated Raman effect occurs. The stimulated Raman effect is the stimulated emission of inelastically scattered light in which photoexcited carriers recombine by coherently emitting inelastically scattered light and the corresponding phonon can be coherent. The difference between stimulated Raman spectroscopy and coherent phonon spectroscopy is that the incident light is not always an ultrafast pulse in stimulated Raman spectroscopy. For an overview of Raman spectroscopy in graphene-related systems, the reader is referred to the literature [25-29].

Multi-wall carbon nanotubes (MWNTs) were discovered by S Iijima who fabricated helical microtubules of graphitic carbon using an arc-discharge evaporation method similar to that used in the synthesis of fullerenes [30]. Transmission microscopy showed that the tubules consisted of coaxial carbon nanotubes ranging in number from about 2 to 50 with the spacing between tubes being approximately $3.4 \AA$ as is typical in graphite materials. SWNTs were later grown using catalysts in arc-discharge generators. Bethune et al found that by vaporizing carbon and a cobalt catalyst in an arc generator, SWNTs with small diameters of around $1.2 \mathrm{~nm}$ could be grown [31]. Iijima et al then grew SWNTs with diameters around $1 \mathrm{~nm}$ using an iron catalyst [32]. Much progress in producing carbon nanotubes has been made since these early pioneering efforts and an excellent overview of various methods for producing carbon nanotubes can be found in [33]. A SWNT can be viewed as a graphene sheet rolled up into a cylinder whose diameter and length are on the order of $1 \mathrm{~nm}$ and $1 \mu \mathrm{m}$, respectively [34]. Because the SWNT energy band structure is one dimensional, a Van Hove singularity in the electronic density of states exists at the $i$ th optical transition energy $E_{i i}$ for light polarized parallel to the SWNT axis [35, 36]. A strong optical absorption at the $E_{i i}$ band edge occurs due to the formation of excitons (bound electron hole pair states) which are stable even at room temperature $[25,37]$. Further we have a low frequency (5 $\mathrm{THz}$ for $1.5 \mathrm{~nm}$ diameter) RBM phonon mode in which the diameter of the SWNT vibrates with a frequency which is inversely proportional of the diameter of the SWNT [38, 39]. Because of the saturation of the photoexcited carriers in low dimensions, the threshold power for observing the stimulated Raman effect becomes quite low $(10 \mathrm{~mW})$ compared with conventional semiconductor materials [40]. Thus SWNTs are an ideal material for observing coherent phonons. 
Graphene nanoribbons are thin strips of planar graphene with straight edges that offer intriguing possibilities for high speed device design. They have been produced by unzipping carbon nanotubes [41] as well as through lithographic [42-44] and chemical means [45]. A detailed review paper on fabrication methods and characterization of GNRs can be found in [46]. GNRs can be thought of as SWNTs that have been unfolded to form a narrow graphene stripe. In GNRs, the electronic structure is also one dimensional and we expect that the coherent RBLM phonon in which the ribbon width vibrates has a large electron-phonon interaction [47].

In coherent phonon spectroscopy, several optical processes occur within 1 ps. First, many photoexcited electron hole pairs or excitons are excited by an ultrafast pump pulse. For high excitation densities, at short time scales ( $<10 \mathrm{fs}$ ), electron-electron scattering causes the carriers to thermalize to an effective electronic temperature that is much higher than the lattice temperature. Some of the excitons emit phonons through the exciton-phonon interaction on $10-100$ fs time scales and initiate coherent phonon vibrations. These coherent phonons can decay via phonon-phonon scattering. The phonon-phonon scattering occurs because of the anharmonicity of the vibrational potential. A typical phonon lifetime is $1-5$ ps. The exciton-phonon scattering matrix elements are small enough so that not all excitons relax by emitting phonons. However, if stimulated Raman effects occur, this is not the case and most of the emitted light is scattered light. The remaining excitons decay non-radiatively by emitting many phonons or radiatively by spontaneous emission of light. Hereafter, we consider only phonon generation by the electron-phonon interaction (i.e. the photoexcited electrons and holes do not interact with each other to form excitons in our treatment) in our discussion of coherent phonons. The exciton-phonon interaction should be a dominant effect in the case of SWNTs. However, since the exciton wavefunction is localized in space, we do not yet have a clear picture of why the localized exciton gives a macroscopic oscillation of $\Delta T / T$ or $\Delta R / R$. However, since the properties of $\Delta T / T$ or $\Delta R / R$ can be obtained by spatially averaging over the wavelength, the localized nature of the exciton might not appear in CP spectroscopy. The treatment of the full exciton-phonon interaction remains for future study.

The optoelectronic properties and characterization of SWNTs have been reviewed from an experimental perspective in the excellent article by Nanot et al in [48]. There they discuss the experimental generation and detection of coherent phonons using $\mathrm{CP}$ spectroscopy and compare the results of CP spectroscopy with resonant Raman spectroscopy (RRS) and photoluminescence excitation (PLE) spectroscopy. It is pointed out that $\mathrm{CP}$ spectroscopy applied to SWNTs has several advantages over continuouswave spectroscopic techniques. In $\mathrm{CP}$ spectroscopy there is no photoluminescence signal or Raleigh scattering background as we discussed earlier. Coherent phonons in an ensemble of micelle suspended SWNTs have been generated by ultrafast laser pulses and detected using femtosecond pump-probe spectroscopy. These coherent phonon spectroscopy experiments measure periodic changes in nanotube optical properties induced by $\mathrm{CP}$ lattice vibrations and, unlike RRS and PLE, allows one to directly measure phonon dynamics, including phase information, in the time domain. The coherent phonon dynamics in micelle suspended nanotubes can also probe details of the interaction of the SWNTs with the environment. Makino et al [49] studied the dynamics of coherent RBM phonons in micelle suspended SWNTs in femtosecond pump-probe impulsive Raman experiments and found a strong environmental $\mathrm{pH}$ dependence in the $\mathrm{CP}$ spectra which they attributed to protonation at the SWNT surface. Using pulse shaping techniques to create a train of pump pulses resonant with the $\mathrm{CP}$ period, one can generate and detect coherent phonons in nanotubes of a specific chirality in an ensemble sample [50]. These resonant CP spectroscopy experiments provide information on the chirality dependence of light absorption, coherent phonon generation, and coherent phonon-induced band structure changes. The lowest frequency coherent phonons that can be photoexcited in SWNTs using ultrafast laser pulses are coherent RBM phonons with phonon wavevector $q=0$ corresponding to a mode in which the diameter of the nanotube periodically expands and contracts. These coherent RBM phonons have been studied experimentally by several groups [51-56]. In addition to the lower frequency coherent RBM modes, higher frequency $q=0$ coherent $\mathrm{G}$ mode phonons have also been observed [57, 58].

We discuss, in this review article, our recent theoretical work on the generation and detection of coherent phonons in carbon SWNTs and GNRs. We focus on the coherent RBM mode in the SWNTs and the coherent RBLM mode in GNRs. We have developed a microscopic theory for the generation and detection of coherent RBM phonons based on an extended (third nearest neighbor) tight-binding (ETB) model for electronic states and a valence force field (VFF) model for the phonons. The microscopic deformation potential electron-phonon interaction in our theoretical model allows us to explain the origin of the chirality and family dependence of the CP spectra observed in SWNTs. Although no experimental results on coherent phonons in GNRs have been reported to date, we have applied our extended tight-binding model to studying CP spectra in armchair edge (aGNR) and zigzag edge (zGNR) graphene nanoribbons. In particular, we focus on the $q=0$ coherent RBLM phonon in which the nanoribbon width periodically expands and contracts.

To further deepen our understanding of coherent phonon phenomena in SWNTs and GNRs, we have studied the generation of coherent RBM and RBLM phonons in these systems using a simpler effective mass theory in which electronic states are treated as Dirac fermions. The electron-phonon interaction in the effective mass theory is treated using Sasaki's deformation-induced gauge field in graphene due to lattice deformations, which gives rise to changes in the nearest neighbor transfer integral in the electronic tight-binding Hamiltonian [59]. Using the effective mass theory, we are able to reproduce most of the results of our extended tight-binding model and explain general 
trends for CP spectroscopy signals. The electron-phonon interaction Hamiltonian in both the effective mass and extended tight-binding theories depends on $k$ in the Brillouin zone and the sign of the interaction determines the initial phase of the RBM CP vibrations which can be observed in CP spectroscopy experiments on SWNTs.

This review article is organized as follows. In section 2 we give a brief introduction and overview of the phenomena of coherent phonons in molecules and solids. In section 3 the basic theory for the generation and detection of CPs in carbon based nanostructures is presented. This includes a discussion of the electronic states and optical properties in sections 3.1 and 3.2, and the phonon modes in section 3.3. In section 3.4 we determine the matrix elements for the deformation potential electron-phonon interaction, both within the tight-binding formalism and using effective mass theory. Using the electron-phonon matrix elements we obtain the driving function in the driven oscillator equation for the coherent phonon amplitude as described in section 3.5. This driving function depends on the photoexcited electron and hole carrier densities, and establishes the connection between the $\mathrm{CP}$ amplitude and the action of the pump laser. The CP amplitude tells us how the carbon atoms oscillate in time and from this we can calculate a time-dependent energy band structure and time-dependent optical properties assuming the electronic structure adiabatically follows the coherent lattice vibrations. The general scheme for detecting CPs from the time-dependent optical prosperities is discussed section 3.6. Coherent phonons in SWNTs are detected by using a delayed probe pulse to measure time-dependent changes in the optical transmission at the probe frequency and we use our calculated optical properties to determine the time-dependent transmission. Finally, the simulated CP signal is calculated by taking the Fourier power spectrum of the theoretical time-dependent transmission at the probe energy. In section 4 we present results for RBM CPs in carbon nanotubes. We compare our theory and calculations with two different types of experiments: (1) micelle suspended nanotubes and (2) films of aligned nanotubes. We then make predictions about the initial phase of the $\mathrm{CP}$ oscillation based on the effective mass theory. In section 5, since no experiments have been performed to date, we present our theoretical calculations and predictions for CPs in zigzag and armchair graphene nanoribbons. The effective mass theory allows us to characterize the initial lattice behavior in armchair nanoribbons and we develop the effective mass $\mathrm{CP}$ theory in zigzag GNRs which is previously unpublished.

\section{Coherent phonons}

The development of ultrafast, femtosecond laser sources has enabled researchers to study dynamical properties of molecular systems, semiconductor nanostructures, and carbon nanotubes [20, 60-66]. Ultrafast femtosecond lasers are ideal for studying electron and hole dynamics since scattering rates typically range from 10 to 100 s of femtoseconds in most semiconductors $[67,68]$.

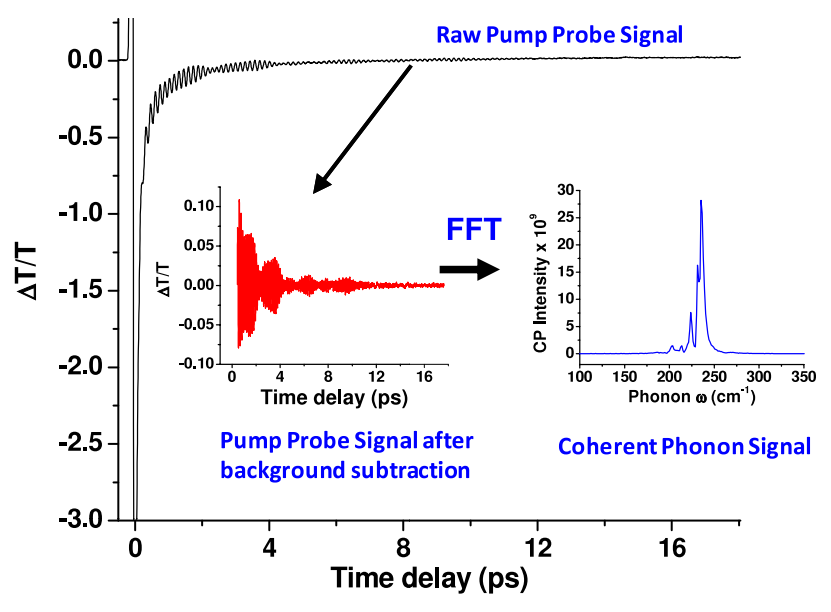

Figure 1. Time-resolved change in transmission $(\Delta T / T)$ of the laser probe pulse as a function of time delay with respect to the pump pulse for a SWNT system. The oscillations superimposed on the background electron and hole relaxation dynamics signal, are known as coherent phonons. To study the coherent phonons, one subtracts off the background carrier dynamics signal and then performs a fast Fourier transform (FFT) to calculate the power spectrum or coherent phonon signal.

A good review of femtosecond spectroscopy, especially in molecular systems, can be found in [69]. The usual femtosecond experiment is a pump-probe experiment where the pump laser beam creates a nonequilibrium distribution of photoexcited electrons and holes. One can study the relaxation dynamics of these nonequilibrium carriers back to equilibrium by measuring the absorption or reflection of the probe pulse as a function of delay time with respect to the pump pulse. The decay or change of the probe signal provides valuable information on the (i) electronic structure, (ii) scattering rates, (iii) relaxation dynamics and mechanisms and (iii) many-body effects in a given material.

In addition to a background decay signal, many systems show oscillating signals superimposed on the carrier dynamics signal. This is illustrated in figure 1, where, in the raw pump-probe signal, we see a fast decay signal (due to the electrons and holes) in $\Delta T / T$ with oscillations on top of this background signal. These oscillations usually match one of the vibrational frequencies of the material and are known as coherent phonons. To better understand the oscillations, one subtracts off the background signal and the performs a Fourier transforms to calculate the power spectrum, which is referred to as the coherent phonon signal. The spectroscopic study of these oscillations is known as coherent phonon spectroscopy.

Coherent phonon spectroscopy can be used to study the dynamics of vibrational motion in a given system. It allows the direct measurement of excited state phonon dynamics in the time domain and includes information on: (1) the phase of the vibration, (2) the electron-phonon coupling, and (3) the decay time of the phonon modes. To excite a given phonon mode however, requires that the ultrashort laser pulse have a duration shorter than the period of the lattice vibration (or the pulse must at least have a Fourier component that matches the vibrational frequency). 


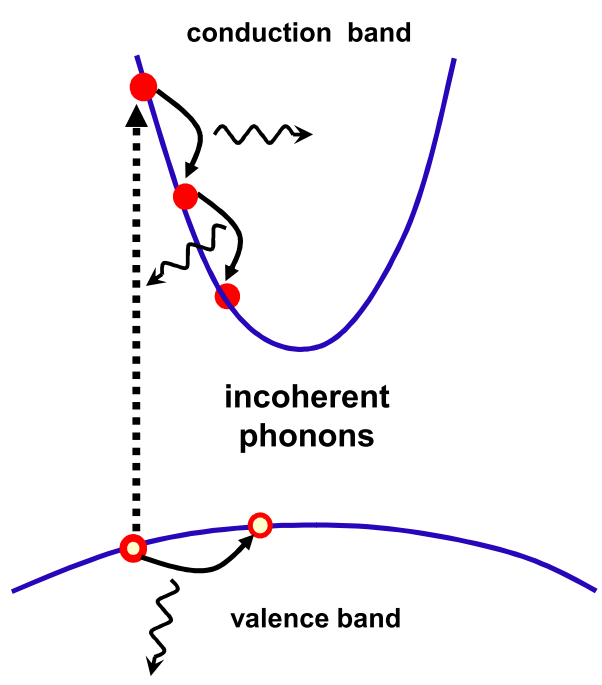

Figure 2. Relaxation of nonequilibrium, photoexcited electrons and holes through optic phonon emission. The phonons that are emitted through the relaxation of the nonequilibrium carries are incoherent phonons.

Coherent phonons should not be confused with the phonons that are generated by the relaxation of the photoexcited, nonequilibrium electrons and holes shown in figure 2. The electrons and holes lose energy and relax to equilibrium through the emission of optic and acoustic phonons. These phonons, however, are defined to be incoherent phonons which are (1) emitted at random times, (2) have no distinct phase relationship, and are not responsible for the oscillations in the pump-probe signal.

How then does one generate coherent phonons? A simple explanation is shown in figure 3 for two prototypical systems: (1) a semiconducting system (GaAs), where the electron-phonon coupling is weak compared to the transfer integral which leads to the energy bands and as a result, the electron-phonon interaction can be treated as a perturbation, and (2) a molecular system where the electron-phonon coupling is strong compared to the transfer integral and one speaks of the combined electronic-vibrational levels.

In a polar semiconductor such as GaAs shown in figure 3(a), there exists a surface depletion field. Before photoexcitation, in response to the surface depletion field, the Ga and As ions are slightly displaced by an amount $x_{1}$ and $x_{2}$ from their equilibrium positions (i.e. with no depletion field inside the semiconductor). After photoexcitation by the pump laser pulse, electrons and holes are photoexcited which create carriers near the semiconductor surface which can (partially) screen out the depletion field. If the photoexcited carriers are created on a fast time scale, the displaced $\mathrm{Ga}$ and As ions want to return to their equilibrium (no depletion electric field) position and trigger the coherent oscillation.

A similar situation is shown in figure 3(b) for a typical molecule. Shown in the figure are the combined electronic and vibrational states for the ground state and the first excited state. The pump pulse creates a rapid photoexcitation of an electron from the ground state energy surface to the excited state energy surface. Since the minimum in the excited state energy surface is at different point than in the ground state energy surface, the rapid photoexcitation to the higher state energy surface triggers the coherent oscillation.

Single wall carbon nanotubes and graphene nanoribbons are interesting because they lie between the simple molecular systems and the extended traditional semiconducting systems. In addition, the electron-phonon coupling in these carbon based nanostructures is not polar like in GaAs. In calculating
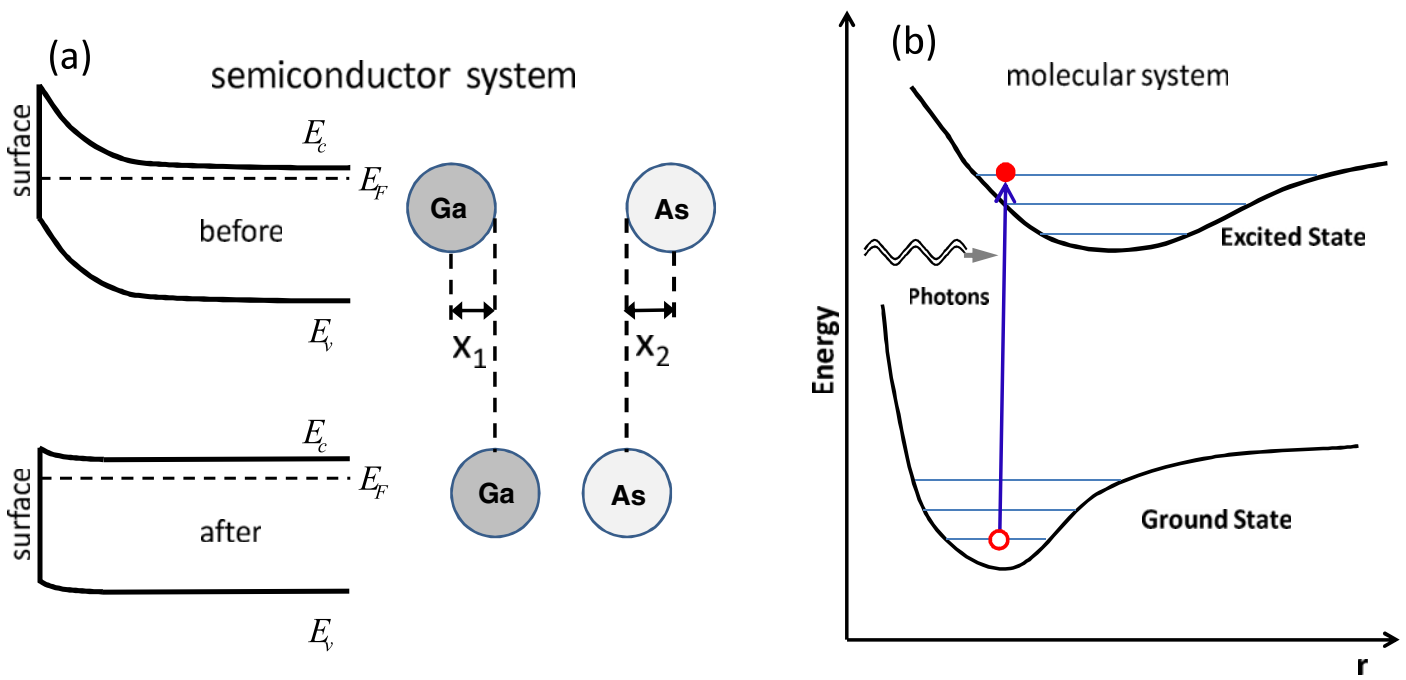

Figure 3. Generation mechanisms for coherent phonons. (a) A conventional polar semiconductor like GaAs. Before photoexcitation by the pump pulse, the surface depletion field cause the Ga and As ions to be displaced by an amount $x_{1}$ and $x_{2}$ from their equilibrium (no depletion field) position. After photoexcitation, the electrons and holes generated near the surface, screen the depletion field causing the Ga and As ions to return to their equilibrium position and trigger the coherent phonon. (b) A typical molecule. Shown are the combined electronic and vibrational energy levels for the grounds state energy surface and an excited state energy surface. Since the ground state and excited state energy surfaces have different minima, photoexcitation by the pump pulse from the ground state energy surface to the excited surface triggers the coherent phonon and the system wants to move to a new minimum. 
and modeling the coherent phonon spectra in carbon nanotubes and graphene, several important effects must be addressed. These include: (i) electronic structure (needed to determine the electron and hole states), (ii) optical matrix elements (needed to determine what states are excited by the pump laser pulse), (iii) phonon modes, (iv) electron-phonon interaction matrix elements (to determine which coherent phonon modes are triggered by the photoexcited electrons) and (v) the generation and detection mechanisms. In the next section, we address these issues for SWNTs and GNRs one by one.

\section{Theory for generation and detection of coherent phonons}

Kuznetsov and Stanton [70] developed a microscopic theory for generation of coherent optical phonons observed in bulk semiconductors excited by ultrafast laser pulses. The coherent optical phonon amplitudes were found to satisfy a driven harmonic oscillator equation (see section 3.5). They found that carriers photoexcited by an ultrafast pump acted as a driving source for coherent optical phonon oscillations via the deformation potential coupling between electrons and phonons.

Dumitrică et al [71, 72] theoretically predicted coherent phonons in finite length carbon nanotubes in nonequilibrium molecular dynamics simulations. These authors treat electric and ionic motion in a tight-binding Hamiltonian with electronic matrix elements and core-core repulsive terms that depend on interatomic distances. The coupling to a classical time-dependent laser field is included and ionic forces are found from the gradient of the electronic free energy. Ultrafast laser excitation resulted in the generation of two coherent phonon modes of different frequencies localized in the cylindrical body and hemispherical end caps of the nanotubes. In combination with ultrafast bond weakening, it was found that the coherent phonon oscillations allowed selective cap opening to take place. This is potentially of interest for nanotechnology applications as it may allow for the controlled manipulation of lattice structures at the nanoscale

Subsequently, a theory of coherent phonons in SWNT excited states has been reported based on tight-binding molecular dynamics simulations [73]. Recently, microscopic theories have been developed for the generation and detection of coherent phonons in SWNTs and GNRs using an extended tight-binding model for electronic states, valence force field models for the phonons, and deformation potential coupling between electrons and phonons [47, 54]. As in bulk non-polar semiconductors, the CP amplitudes in SWNTs and GNRs are found to satisfy a driven oscillator equation with the driving term depending on the photoexcited carrier densities. The results of these extended tight-binding theories have been confirmed using effective mass electronic states, phonon modes, and nearest neighbor deformation potential electron-phonon interactions [47, 74]. In the following subsections we describe the elements of these microscopic theories in more detail.

\subsection{Electronic states}

The length of a typical SWNT is many times larger than the tube diameter. It is a good approximation to consider SWNTs of infinite length which can be thought of as rolled up sheets of graphene. The way the graphene sheet is wrapped to form a SWNT is specified by the roll up or chiral vector $\mathbf{C}_{h}=n \mathbf{a}_{1}+$ $m \mathbf{a}_{2} \equiv(n, m)$ where $\mathbf{a}_{1}=a / 2(\sqrt{3}, 1)$ and $\mathbf{a}_{2}=a / 2(\sqrt{3},-1)$ are the unit cell vectors of the graphene hexagonal lattice and $n$ and $m$ are the chirality indices. For graphene, the hexagonal lattice constant $a=2.49 \AA$ is related to the carbon-carbon bond length $a_{\mathrm{C}-\mathrm{C}}=1.44 \AA$ by $a=\sqrt{3} a_{\mathrm{C}-\mathrm{C}}$. In the SWNT the roll up vector circles the tube circumference with the head of the rolled up vector touching the tail. The pair of chirality indices $(n, m)$ with $0 \leq m \leq n$ uniquely specify the structure of the infinite ideal SWNT. If $m=0$ the SWNT is referred to as a zigzag nanotube, if $m=n$ the SWNT is referred to as an armchair nanotube, and if $0<m<n$ the SWNT is referred to as a chiral nanotube $[34,75]$.

Because of translational symmetry along the nanotube axis, SWNTs have a one-dimensional band structure with unique electronic properties. They can be either metallic or semiconducting depending on their chirality indices $(n, m)$ [7, 34, 76-79]. A widely used description of the electronic band structure of SWNTs is a nearest neighbor empirical tight-binding model that includes only the $\pi$ orbitals perpendicular to the nanotube surface since these give rise to the electronic bands close to the Fermi level [34].

We note that in calculating electronic bands, one can also consider the hybridized $\operatorname{sp}^{2} \sigma$ bands which are responsible for the strong bonding between the carbon atoms [34]. In planar graphene and GNRs, the $\pi$ and $\sigma$ states do not couple thanks to the planar symmetry in ideal graphene and GNRs. However, curvature in fullerenes and carbon nanotubes causes the $\pi$ and $\sigma$ bands to hybridize. Fortunately, this hybridization is weak enough to be ignored in SWNTs with diameters larger than $0.6 \mathrm{~nm}$ [75]. For tubes with diameters less than $0.6 \mathrm{~nm}$, the hybridization can be strong enough to alter the electronic states. In particular, small diameter tubes that are predicted to be semiconducting in the absence of $\sigma-\pi$ hybridization in tight-binding calculations are found to be metallic in $a b$ initio calculations that take $\sigma-\pi$ hybridization into account [80].

Samsonidze et al [81] have calculated electronic energy bands and optical transition energies in small diameter SWNTs in the extended tight-binding (ETB) model of Porezag et al [82] taking $\sigma-\pi$ hybridization into account. For SWNTs whose diameters are not too small, we ignore $\sigma-\pi$ hybridization and treat the $\pi$ and $\pi^{*}$ electronic states in SWNTs using the above mentioned ETB model for the $\pi$ bands. However, in our ETB model, we do not include the curvature induced $\sigma-\pi$ hybridization. In the Porezag et al [82] ETB model for carbon atoms, the tight-binding Hamiltonian and overlap matrix elements between carbon $\pi$ orbitals on different atoms are functions of the interatomic distance. Position-dependent Hamiltonian and overlap matrix elements are obtained by parameterizing density-functional (DFT) results in the local-density approximation (LDA) for a wide selection of carbon compounds [82]. These 


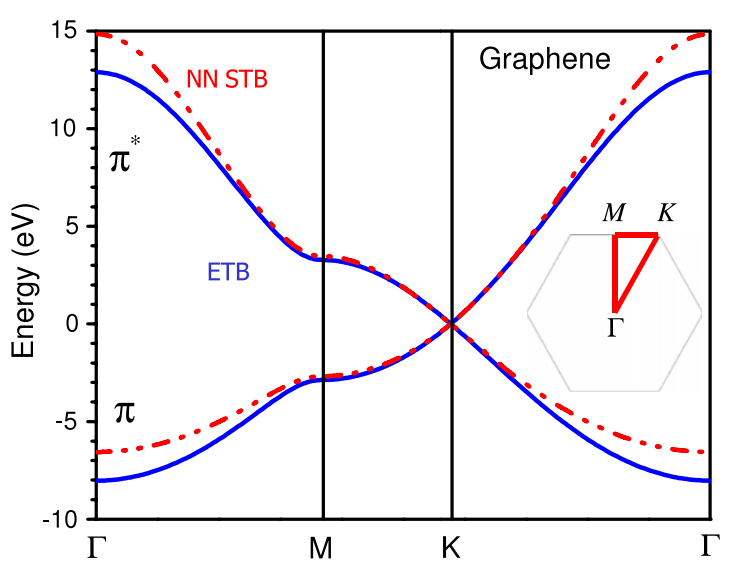

Figure 4. Energy dispersion relations for the bonding $\pi$ and anti-bonding $\pi^{*}$ bands in graphene along high symmetry directions in the hexagonal Brillouin zone. The calculated bands in forth nearest neighbor extended tight-binding model are shown as solid blue lines and the bands obtained with the nearest neighbor simple tight-binding model described in [34] are shown as red dash-dotted curves. The high symmetry lines are shown schematically in the inset where the Brillouin zone has been rotated clockwise by $30^{\circ}$.

parameterized matrix elements are transferable to a wide range of carbon compounds and vanish beyond third nearest neighbor distances. The third nearest neighbor ETB has the advantage that it can be used to compute time-dependent adiabatic changes in the electronic structure due to changes in the lattice induced by $\mathrm{CP}$ lattice vibrations. The computed energy bands in graphene are plotted in figure 4 along high symmetry directions in the two-dimensional hexagonal Brillouin zone shown schematically in the inset. The bands calculated in the third neighbor ETB model are shown as solid blue lines and the results of the nearest neighbor simple tight-binding model described in [34] are shown as red dash-dotted lines.

In a SWNT with chiral indices $(n, m)$, a translational vector $\mathbf{T}$ can be found parallel to the tube axis $[34,75]$. The translational vector is $\mathbf{T}=t_{1} \mathbf{a}_{1}+t_{2} \mathbf{a}_{2} \equiv\left(t_{1}, t_{2}\right)$ where $t_{1}=$ $(2 m+n) / \operatorname{gcd}(2 n+m, 2 m+n)$ and $t_{2}=-(2 n+m) / \operatorname{gcd}(2 n+$ $m, 2 m+n)$ with $\operatorname{gcd}(i, j)$ being the greatest common divisor of two positive integers $i$ and $j$. For the resulting one-dimensional Brillouin zone, $|k| \leq \pi / T$. Retaining one $\pi$ orbital per atomic site and exploiting the translational symmetry, the resulting size of the Hamiltonian and overlap matrices is $2 N_{\text {hex }} \times 2 N_{\text {hex }}$, where $N_{\text {hex }}=2\left(n^{2}+n m+m^{2}\right) / \operatorname{gcd}(2 n+m, 2 m+n)$ is the number of two-atom hexagonal cells in the SWNT unit cell defined by $\mathbf{C}_{h}$ and $\mathbf{T}$. As pointed out in [83], we can make use of the screw symmetry operations to block diagonalize the $2 N_{\text {hex }} \times 2 N_{\text {hex }}$ Hamiltonian and overlap matrices into $2 \times 2$ subblocks which we label $\mu$. In SWNTs, the subblock index $\mu$ labels the cutting lines in the zone folding picture so the cutting line indices are good quantum numbers for SWNT $\pi$ bands.

The unit cell of a SWNT lies in the curved surface area defined by the chiral vector $\mathbf{C}_{h}$ in the circumferential direction and the translation vector $\mathbf{T}$ along the tube axis. The nanotube reciprocal lattice vectors $\mathbf{K}_{1}$ along the circumferential direction and $\mathbf{K}_{2}$ parallel to the tube axis are
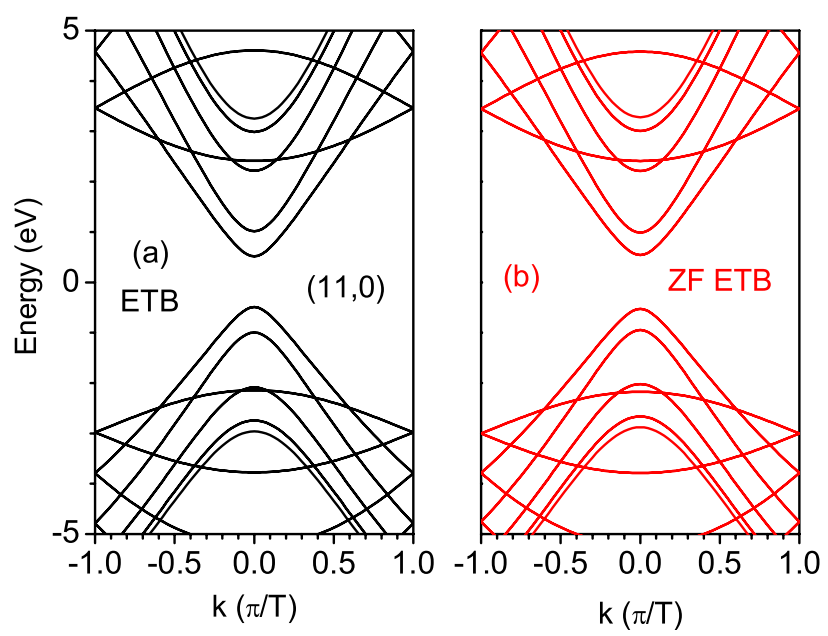

Figure 5. (a) Electronic $\pi$ bands for the $(11,0)$ zigzag SWNT calculated in the third nearest neighbor ETB model and (b) the same bands obtained from the zone folding picture equation (1).

obtained from the relations $\mathbf{C}_{h} \cdot \mathbf{K}_{1}=\mathbf{T}_{h} \cdot \mathbf{K}_{2}=2 \pi$ and $\mathbf{C}_{h} \cdot$ $\mathbf{K}_{2}=\mathbf{T} \cdot \mathbf{K}_{1}=0$. From these relations the nanotube reciprocal lattice vectors are found to be $\mathbf{K}_{1}=\left(-t_{2} \mathbf{b}_{1}+t_{1} \mathbf{b}_{2}\right) / N_{\text {hex }}$ and $\mathbf{K}_{2}=\left(m \mathbf{b}_{1}-n \mathbf{b}_{2}\right) / N_{\text {hex }}$ where $\mathbf{b}_{1}=(2 \pi / a)(1 / \sqrt{3}, 1)$ and $\mathbf{b}_{2}=(2 \pi / a)(1 / \sqrt{3},-1)$ are the reciprocal lattice vectors for graphene $[34,75]$. Because of the translational symmetry along the tube axis, the tube has a one-dimensional Brillouin zone $-\pi / T \leq k \leq \pi / T$ along the tube axis. The wavevector in the circumferential direction is quantized by the periodic boundary condition. In the zone folding picture, the nanotube electronic states for the $\mu$ th cutting line in a SWNT is given by [34]

$$
\begin{aligned}
E_{\mu}^{s}(k) & =E_{2 \mathrm{D}}^{s}\left(k \frac{\mathbf{K}_{2}}{\left|\mathbf{K}_{2}\right|}+\mu \mathbf{K}_{1}\right), \\
(\mu & \left.=0 \cdots N_{\mathrm{hex}}-1,|k| \leq \pi / T\right),
\end{aligned}
$$

where $s=\mathrm{c}, \mathrm{v}$ labels the conduction and valence $\pi$ bands and $E_{2 \mathrm{D}}^{s}(\mathbf{k})$ are the graphene ETB electronic bands in the 2D Brillouin zone plotted in figure 4 along high symmetry directions. The $\mu$ th $1 \mathrm{D}$ cutting line in the $2 \mathrm{D}$ Brillouin zone is given by the argument of $E_{2 \mathrm{D}}^{s}(\mathbf{k})$ in equation (1).

Figures 5 and 6 show the computed electronic $\pi$ bands for the zigzag $(11,0)$ and chiral $(8,6)$ semiconducting SWNTs in the $2 n+m=22$ family of nanotubes. The figures show the effects of chirality on the electronic structure. Note that owing to the large number of bands for the $(8,6)$ SWNTs, the energy scale only goes from -3 to $+3 \mathrm{eV}$. The results of the full ETB model are shown in (a) as solid black lines and the corresponding zone folded (ZF ETB) bands are shown in (b) as solid red lines. Comparing the ETB and ZF ETB band structures, we see that the zone folded bands give remarkably accurate results. This is due to the fact that (i) bands belonging to different cutting lines do not mix thanks to the SWNT screw symmetries and to the fact that (ii) conduction and valence band states belonging to the same cutting line do not cross. 

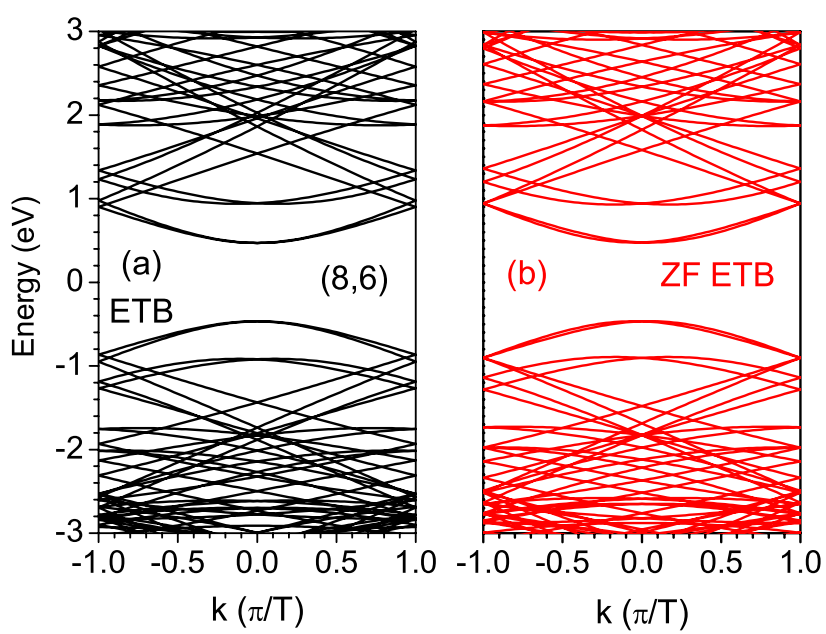

Figure 6. (a) Electronic $\pi$ bands for the $(8,6)$ chiral SWNT calculated in the third nearest neighbor ETB model and (b) the same bands obtained from the zone folding picture equation (1). Note that the energy scale in this figure differs from figure 5.

\subsection{Optical properties}

The absorption coefficient at photon energy $\hbar \omega$ is given by $[84,85]$

$$
\alpha(\hbar \omega)=\frac{\hbar \omega}{n_{\mathrm{g}} \hbar c} \varepsilon_{2}(\hbar \omega),
$$

where $\varepsilon_{2}(\hbar \omega)$ is the imaginary part of the dielectric function evaluated at the photon energy $\hbar \omega$, and $n_{\mathrm{g}}$ is the index of refraction. We calculate the absorption coefficient by evaluating the imaginary part of the dielectric function in the dipole approximation using Fermi's golden rule. Similar formulas can be derived for graphene, SWNTs and GNRs. For zigzag and armchair GNRs, the Fermi golden rule imaginary dielectric function is given in [47]. For SWNTs we have [54]

$$
\begin{aligned}
\varepsilon_{2}(\hbar \omega)= & \frac{8 \pi^{2} e^{2}}{A_{\mathrm{t}}(\hbar \omega)^{2}}\left(\frac{\hbar^{2}}{m_{0}}\right) \sum_{n n^{\prime}} \int \frac{\mathrm{d} k}{\pi}\left|P_{n n^{\prime}}(k)\right|^{2} \\
& \times\left(f_{n}(k)-f_{n^{\prime}}(k)\right) \delta\left(E_{n^{\prime}}(k)-E_{n}(k)-\hbar \omega\right),
\end{aligned}
$$

where $A_{\mathrm{t}}=\pi\left(d_{\mathrm{t}} / 2\right)^{2}$ is the cross sectional area of the tube and the tube diameter is $d_{\mathrm{t}}=(a / \pi) \sqrt{n^{2}+n m+m^{2}}$. The electron distribution functions are $f_{n}(k)$, the electronic band energies are $E_{n}(k)$, and the squared optical matrix elements for optical transitions between the tight-binding electronic states $|n k\rangle$ and $\left|n^{\prime} k\right\rangle$ are $\left|P_{n n^{\prime}}(k)\right|^{2}$. We replace the delta function in equation (3) by a broadened Lorentzian lineshape with a FWHM of $\Gamma_{s}$. The optical dipole matrix element for vertical transitions between states $|n k\rangle$ and $\left|n^{\prime} k\right\rangle$ is $P_{n n^{\prime}}(k)=$ $\hbar / \sqrt{2 m_{0}} \hat{\mathbf{e}} \cdot\left\langle n^{\prime} k|\nabla| n k\right\rangle$ where $\hat{\mathbf{e}}$ is the unit electric polarization vector.

Figure 7 shows the squared optical matrix elements for dipole transitions between the valence and conduction $\pi$ bands in planar graphene for linearly polarized light. Squared optical matrix elements are shown for linear polarization angles ranging from $0^{\circ}$ to $90^{\circ}$ in $30^{\circ}$ increments. To get a feel for the optical properties in carbon materials obtained using Fermi's golden rule, we calculate the optical properties of planar graphene for circularly polarized light incident normally to an infinite graphene sheet. For our ETB model, the $\pi$ bands are shown in figure 4 and the resulting joint density of states (JDOS) as a function of transition energy is
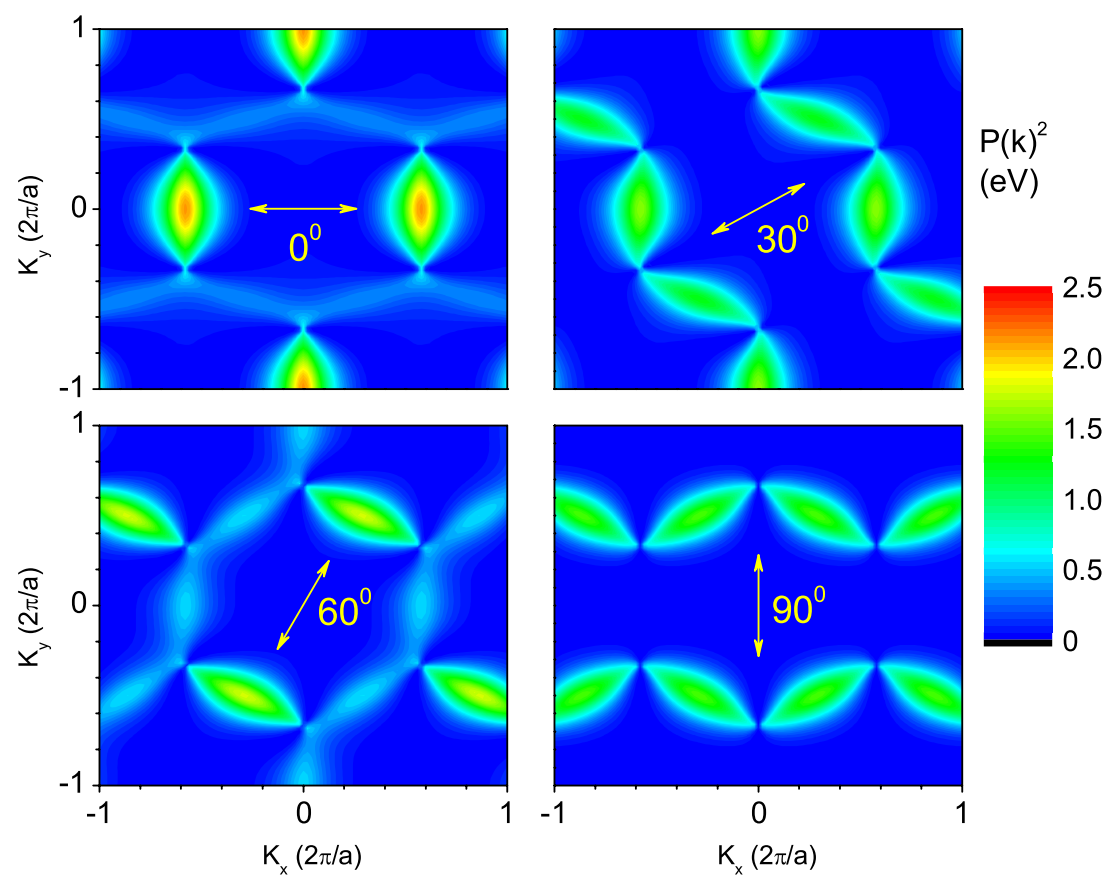

Figure 7. Squared optical matrix element for dipole allowed transitions between valence and conduction $\pi$ bands in graphene in linearly polarized light. Four different linear polarization angles are shown from $0^{\circ}$ to $90^{\circ}$. 


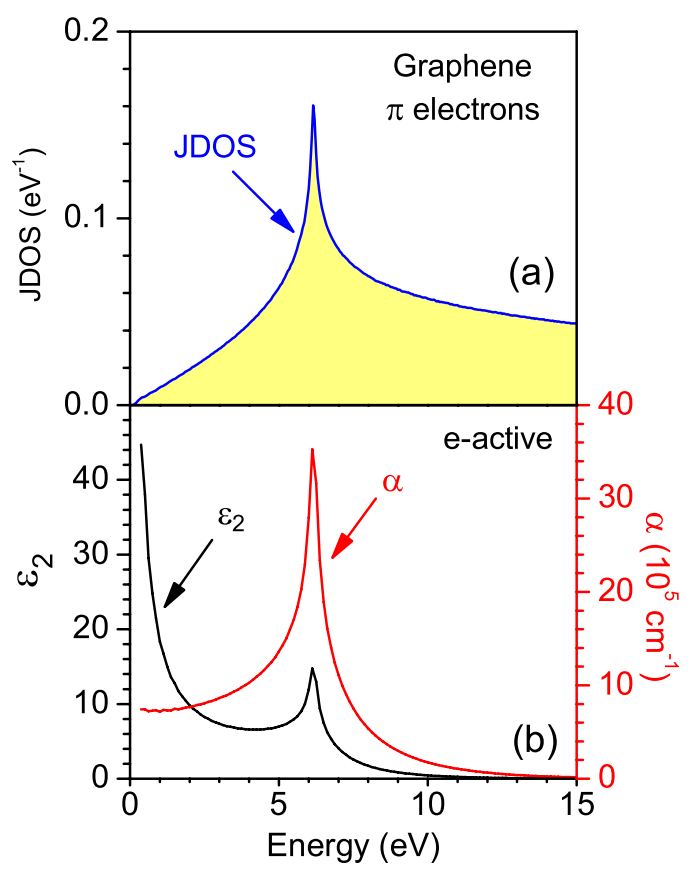

Figure 8. ETB calculations in planar graphene for (a) joint density of states (states/unit cell/eV) and (b) imaginary dielectric function $\varepsilon_{2}$ and absorption coefficient $\alpha$ for normally incident e-active (positive helicity $\sigma^{+}$) circularly polarized light.

plotted in figure 8(a). At $6.1 \mathrm{eV}$ the JDOS has a logarithmic singularity corresponding to transitions at the $\mathrm{M}$ point in the Brillouin zone where the $E_{\mathrm{c}}(\mathbf{k})-E_{\mathrm{V}}(\mathbf{k})$ surface in $\mathbf{k}$ space has a saddle point. This agrees with the tight-binding results of Pedersen et al [86] who also obtain a peak in the JDOS at $6.1 \mathrm{eV}$. Our theoretical JDOS has an M point logarithmic singularity at $6.1 \mathrm{eV}$ while the observed JDOS peak occurs near $4.4 \mathrm{eV}$ [87].

The imaginary part of the dielectric function $\varepsilon_{2}(\hbar \omega)$ and the absorption coefficient $\alpha(\hbar \omega)$ as a function of photon energy $\hbar \omega$ are shown in figure 8(b) for normally incident e-active circularly polarized light (positive helicity $\sigma^{+}$). We used a Gaussian FWHM linewidth of $0.1 \mathrm{eV}$. In the limit $\hbar \omega \rightarrow 0$ the imaginary part of the dielectric function diverges as $1 / \hbar \omega$. This is in agreement with the results of Pedersen et al [86] who evaluated the dielectric function analytically in a nearest neighbor tight-binding model using the expression of Ehrenreich and Cohen [88] for the complex dielectric function. From this result and equation (2) it follows that $\alpha(\hbar \omega)$ approaches a constant as $\hbar \omega \rightarrow 0$. Above the logarithmic singularity, the absorption coefficient drops off sharply with increasing energy. This can be attributed to quenching of the squared optical matrix element near the $\Gamma$ point.

The symmetric peak in the absorption spectrum due to $\mathrm{M}$ point transitions is predicted to occur at $6.1 \mathrm{eV}$ while the observed absorption spectrum is peaked near $4.4 \mathrm{eV}$ and is highly asymmetric. Part of the discrepancy is due to the fact that our model neglects Coulomb interactions between photoexcited electrons and holes and between photoexcited electrons and valence electrons, which we call the exciton binding energy and electron self-energy, respectively. Yang

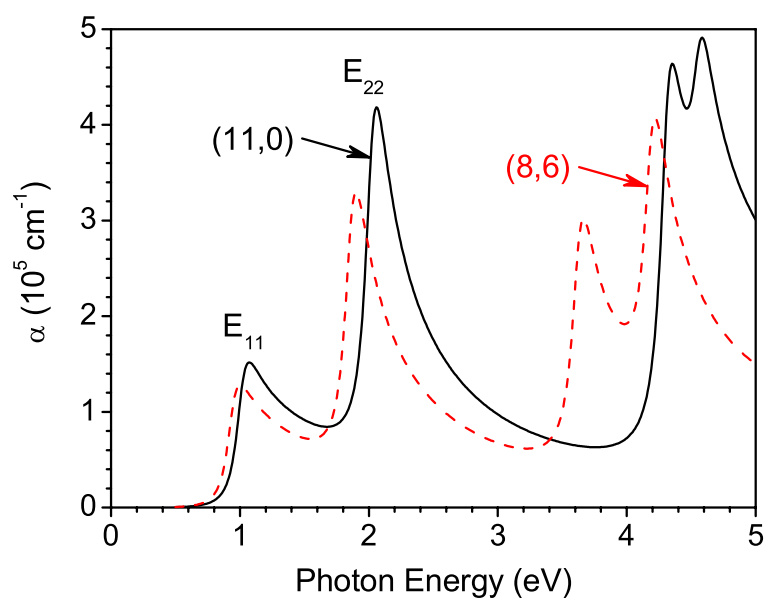

Figure 9. Absorption coefficient for the $(11,0)$ (solid black line) and $(8,6)$ (dashed red line) SWNTs as functions of photon energy for light linearly polarized parallel to the tube axis.

et al [89] theoretically studied the absorption spectrum in graphene using the many-body GW-Bethe Salpeter equation. In their calculation, resonant excitons give rise to a highly asymmetric peak in the absorption at $4.5 \mathrm{eV}$. This peak is redshifted to lower energy by $0.6 \mathrm{eV}$ relative to the absorption peak obtained by Yang et al in the independent particle picture.

The absorption coefficients obtained with Fermi's golden rule using equations (3) and (2) are shown in figure 9 for the semiconducting $(11,0)$ and $(8,6)$ SWNTs for light linearly polarized parallel to the tube axis. The FWHM linewidth is taken to be $0.15 \mathrm{eV}$. The squared optical matrix elements are slowly varying near the band edge, so the absorption coefficients are roughly proportional to the joint density of states for the allowed transitions and are sharply peaked by the van Hove singularities at the direct band gaps. The lowest two peaks are labeled $E_{11}$ and $E_{22}$. The absorption coefficients calculated in figure 9 do not include many-body Coulomb interaction effects. When these are included strong excitonic peaks occur near the absorption band edges and strongly modify the optical properties [90-92].

\subsection{Phonon modes}

Phonons in graphene, SWNTs and GNRs have been studied by a number of techniques including elastic continuum models [93-99], valence force field models [34, 100-103] bond charge models [104], and ab initio methods [105-110].

In our work, we treat phonon dispersion relations in planar graphene using a valence force field model [34]. We include radial $(r)$ bond-stretching interactions as well as transverse in-plane ( $t i$ ) and out-of-plane (to) bond bending interactions. The force constants for these interactions are denoted $\phi_{r}^{(n)}, \phi_{t i}^{(n)}$, and $\phi_{t o}^{(n)}$ respectively where the integers $n=1-4$ label the nearest neighbor atomic shells surrounding each carbon atom. As pointed out in [34], we must include at least fourth neighbor interactions to describe the bond twisting interaction involving a carbon-carbon $\mathrm{sp}^{2}$ bond and the four attached carbon-carbon bonds with a grand total of six carbon 

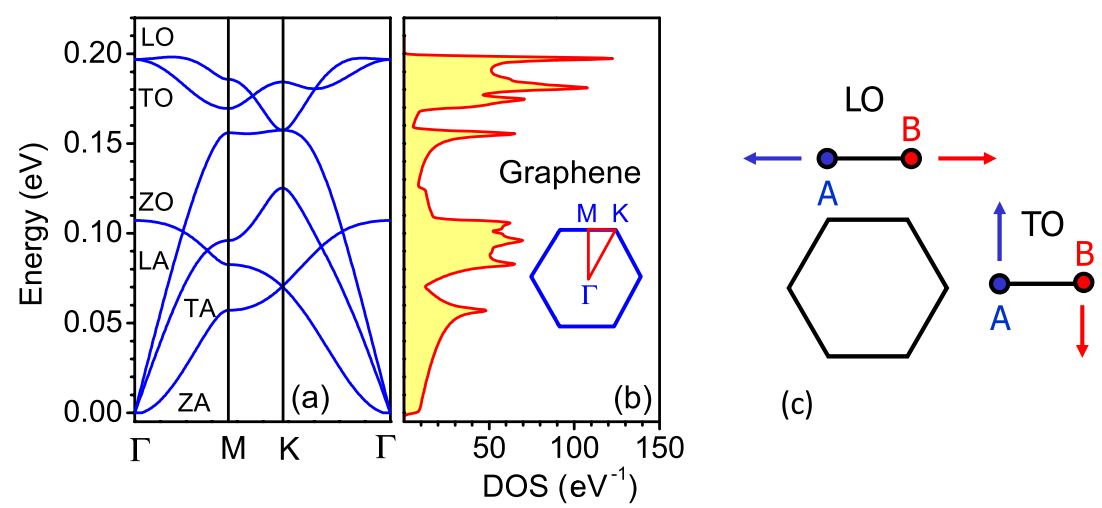

(c)

Figure 10. (a) Graphene phonon energies, $\hbar \omega(\mathbf{q})$, along high symmetry lines in the hexagonal Brillouin zone. (b) Phonon density of states in units of phonon modes per hexagonal unit cell per electronvolt. The high symmetry lines are shown schematically in the inset where the Brillouin zone has been rotated clockwise by $30^{\circ}$. In (c) the mode displacement vectors for the $\mathbf{q}=0$ in-plane LO and TO phonons are shown schematically.

atoms. The most widely separated of these six carbon atoms are separated by the fourth neighbor distance. We use 12 force constant values obtained from fits to experimental data [100] keeping up to fourth neighbor interactions. In graphene, there are two atoms per hexagonal unit cell giving rise to six phonon branches. The phonon energies $\hbar \omega(\mathbf{q})$ and corresponding mode displacement vectors are obtained by diagonalizing a $6 \times 6$ dynamical matrix given in [34] .

The graphene phonon dispersion relations are shown in figure 10(a) where phonon energy is plotted along high symmetry lines in the hexagonal Brillouin zone. There are six phonon modes. The corresponding density of states for the phonon modes in units of modes per hexagonal unit cell per $\mathrm{eV}$ is shown in figure $10(\mathrm{~b})$. Near the $\Gamma$ point $(\mathbf{q}=0)$, there are three acoustic and three optical branches. The lowest acoustic branch is an out-of-plane transverse mode (ZA) whose energy varies as $\mathbf{q}^{2}$. There are two in-plane acoustic modes with energies varying linearly as $|\mathbf{q}|$. The lower lying of these two modes is a transverse acoustic mode (TA) and the higher lying mode is a longitudinal acoustic mode (LA). The lowest lying optical branch is an out-of-plane transverse mode $(\mathrm{ZO})$ with a negative $\mathbf{q}^{2}$ energy dependence at the $\Gamma$ point. The remaining two optical branches are in-plane transverse optical (TO) and longitudinal optical (LO) modes which are degenerate at the $\Gamma$ point and whose energy dependence is approximately constant for small values of $\mathbf{q}$. For the $\Gamma$ point LO mode the A and $\mathrm{B}$ atoms vibrate parallel to the graphene $\hat{x}$ axis (parallel to the bond connecting the $\mathrm{A}$ and $\mathrm{B}$ atoms) $180^{\circ}$ out of phase with each other. For the $\Gamma$ point TO mode the atoms vibrate out of phase with each other parallel to the graphene $\hat{y}$ axis. The mode displacement vectors for the LO and TO modes are shown schematically in figure $10(\mathrm{c})$.

The above valence force field model works well for graphene and planar carbon structures such as GNRs. However, in SWNTs where curvature affects are important care must be taken to ensure that the force constant sum rule is obeyed [111]. This simply means that the valence force field potential energy terms must be invariant under rigid translations and rigid rotations of the nanotube about the nanotube axis. In [102], Mahan and Jeon pointed out that many calculations in the literature use force field models that violate the force constant sum rule and fail to reproduce long wavelength flexure modes predicted by elasticity theory. To remedy this problem in our calculations, we follow Jiang et al [112] and Lobo et al [101] and treat lattice dynamics in carbon nanotubes using a modified valence force field model (MVFF) in which the force constant sum rule is obeyed so that the force field potentials are invariant under rigid translations and rotations. In our MVFF model, we include bond stretching, in-plane bond bending, out-of-plane bond bending, and bond twisting potentials. Our MVFF model for SWNTs has seven force constants [54], four due to bond-stretching interactions out to fourth nearest neighbor shells and one each from the remaining three interactions. We obtained force constants for the MVFF model by fitting our MVFF results for graphene to the VFF results shown in figure 10. Figure 11 shows the best fit MVFF results as red solid lines and the VFF model results as black dots. In the fitting procedure, we gave added emphasis to the low frequency phonons. In what follows, we will use the VFF model in graphene and GNRs and the MVFF model for SWNTs. The force constants in our phonon models are independent of the density of photoexcited carriers and cannot describe phonon softening observed at high values of the laser fluence.

Figure 12 shows the computed phonon dispersion relations for the zigzag $(11,0)$ and chiral $(8,6)$ semiconducting SWNTs. Because of the SWNT screw symmetries, the phonon dynamical matrix can be block diagonalized into $6 \times 6$ submatrices each of which corresponds to a different value of the cutting line index $\mu=0, \ldots, N_{\text {hex }}-1$. The CP active phonon modes are $q=0$ modes with nonzero frequency and cutting line index $\mu=0$. The $\mathrm{CP}$ active mode with the lowest frequency is the RBM mode. In figure 12 the $\mu=0$ acoustic phonon branches are shown as blue lines while the $\mu=0$ branches containing the $q=0$ radial breathing mode (RBM) are shown as thick red lines.

\subsection{Electron-phonon matrix elements}

The deformation potential electron-phonon interaction between carriers photoexcited by ultrafast laser pulses and the 


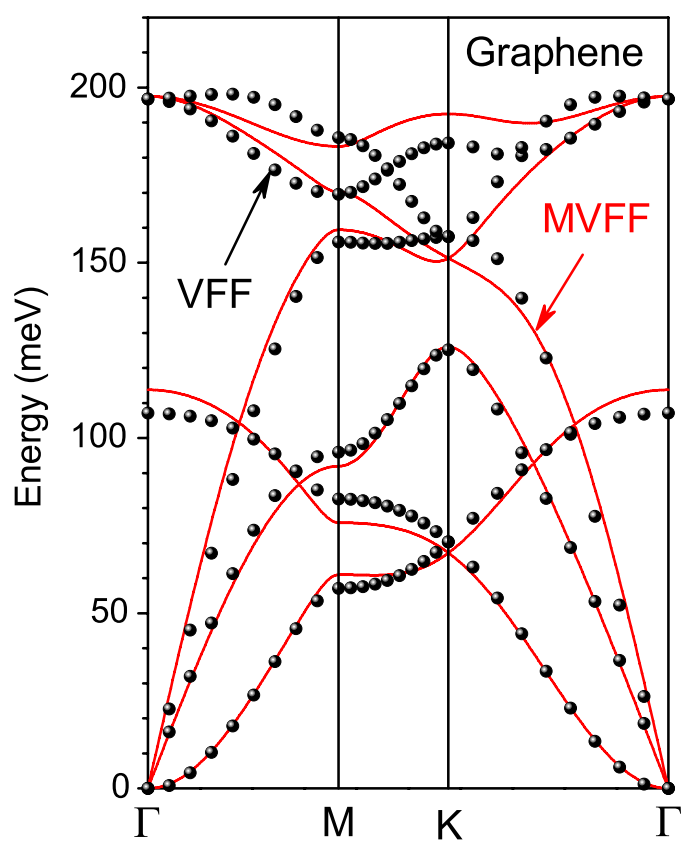

Figure 11. Graphene phonon energies, $\hbar \omega(\mathbf{q})$, along high symmetry lines in the hexagonal Brillouin zone. Black dots are obtained using the 12 parameter valence force field model (VFF) described in the text and the solid red curves are the best fit phonon energies for the 7 parameter modified valence force field model (MVFF). The MVFF fits are optimized for low phonon energies.

phonon modes is responsible for the generation of coherent phonons in non-polar semiconductors as well as carbon materials and nanostructures. In modeling the deformation potential electron-phonon interaction, we use two different models in our ETB and effective mass coherent phonon theories. Here we discuss the electron-phonon interaction for SWNTs.

3.4.1. Extended tight-binding model. In the ETB model, the deformation potential electron-phonon interaction Hamiltonian in a SWNT is given by

$$
H_{\mathrm{ep}}(\mathbf{r})=-\sum_{s j l} \nabla v\left(\mathbf{r}-\mathbf{R}_{s j}^{l}\right) \cdot \mathbf{U}_{s j}^{l}
$$

where $s=\mathrm{A}$, B labels the A and B sublattices, $j=$ $1, \ldots, 2 N_{\text {hex }}$ labels the carbon atoms in a unit cell, and $l=-\infty, \ldots, \infty$ labels the translational unit cell. The equilibrium carbon atomic positions are $\mathbf{R}_{s j}^{l}$, the corresponding displacements from equilibrium are $\mathbf{U}_{s j}^{l}$, and $v(\mathbf{r})$ is the screened atomic potential.

The electron field operator $\hat{\psi}(\mathbf{r})$ is

$$
\hat{\psi}(\mathbf{r})=\sum_{n k} c_{n k} \psi_{n k}(\mathbf{r}),
$$

where $\psi_{n k}(\mathbf{r})$ is the tight-binding wavefunction for the $n$th state with wavevector $k$. The annihilation operator for this state is $c_{n k}$ and we obtain the second-quantized ETB electron-phonon interaction by evaluating the integral

$$
\hat{H}_{\mathrm{ep}}=\int \mathrm{d} \mathbf{r} \hat{\psi}^{\dagger}(\mathbf{r}) H_{\mathrm{ep}}(\mathbf{r}) \hat{\psi}(\mathbf{r})
$$

and replacing the classical atomic displacements $\mathbf{U}_{s j}^{l}$ with the second-quantized phonon operators

$$
\mathbf{U}_{s j}^{l}=\frac{\hbar}{\sqrt{2 \rho L_{\Omega}}} \sum_{m q} \frac{\hat{\mathbf{e}}_{s j}^{m}(q)}{\sqrt{\hbar \omega_{m}(q)}} \mathrm{e}^{\mathrm{i} q T l}\left(b_{m q}+b_{m,-q}^{\dagger}\right) .
$$
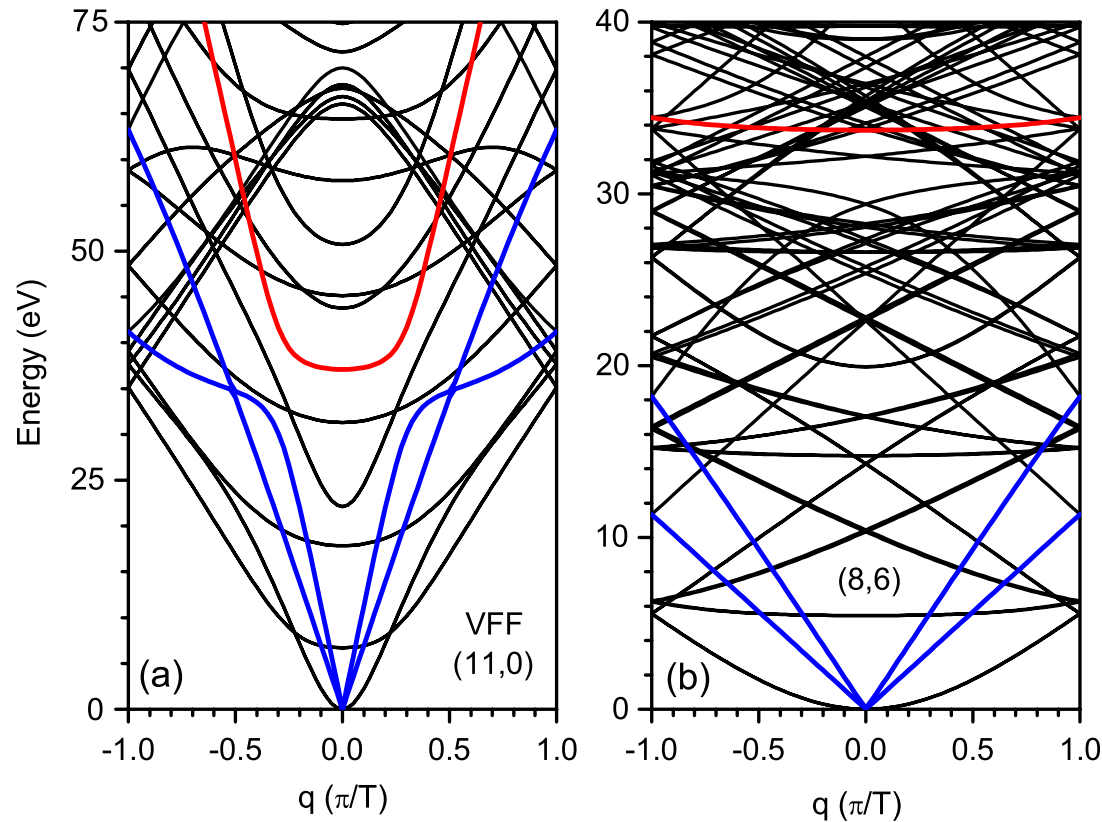

Figure 12. Phonon dispersion relations for (a) the $(11,0)$ zigzag SWNT and (b) the $(8,6)$ chiral SWNT calculated using the modified valence force field model. The acoustic modes are thick blue lines and the radial breathing mode (RBM) is shown as a thick red line. 


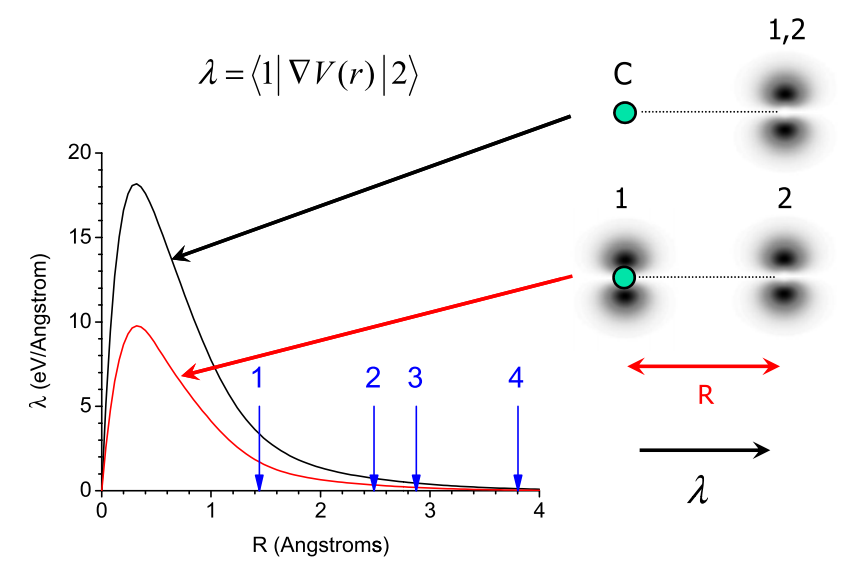

Figure 13. Two-center deformation potential vectors $\vec{\lambda}$ as a function of atomic separation involving two $2 \mathrm{p}_{z}(|1\rangle$ and $|2\rangle)$ electrons and a central carbon atom with screened potential $V(\mathbf{r})$. The vector $\vec{\lambda}$ points from a central carbon atom $C$ on the left to the neighboring carbon atom in question. The atomic distances from a central atom to the first four neighbor shells are indicated. The two possible two-center configurations are shown on the right. For the upper black curve, both electrons sit on the neighboring site and for the lower red curve, one electron sits on each carbon atom.

Here $\rho$ is the mass density per unit length and $L_{\Omega}$ is the sample length. The phonon mode displacement vectors $\mathbf{e}_{s j}^{m}(q)$ are eigenvectors of the dynamical matrix for the phonon modes.

The second-quantized electron-phonon interaction is now

$$
\hat{H}_{\mathrm{ep}}=\sum_{m} \sum_{n^{\prime} k^{\prime} ; n k} M_{n^{\prime} k^{\prime} ; n k}^{m q} c_{n^{\prime} k^{\prime}}^{\dagger} c_{n k}\left(b_{m q}+b_{m,-q}^{\dagger}\right),
$$

where $q \equiv k^{\prime}-k$. The interaction matrix element is given by

$$
\begin{aligned}
M_{n^{\prime} k^{\prime} ; n k}^{m q}= & -A_{m}(q) \sum_{s j ; s^{\prime} j^{\prime}} C_{s^{\prime} j^{\prime}}^{*}\left(n^{\prime} k^{\prime}\right) C_{s j}(n k) \\
& \times \sum_{l l^{\prime} ; s^{\prime \prime} j^{\prime \prime}} \mathrm{e}^{\mathrm{i} T\left(k l-k^{\prime} l^{\prime}\right)} \hat{\mathbf{e}}_{s^{\prime \prime} j^{\prime \prime}}^{m}(q) \cdot \vec{\lambda}\left(s^{\prime} j^{\prime} l^{\prime} ; s^{\prime \prime} j^{\prime \prime} ; s j l\right),
\end{aligned}
$$

where $A_{m}(q)=\hbar / \sqrt{2 \rho L_{\Omega} \hbar \omega_{m}(q)}$ is the quantized phonon amplitude. In equation (9) $\mathrm{T}$ is the length of the translational unit cell, $C_{s j}(n k)$ are the electronic state tight-binding expansion coefficients, $\hat{\mathbf{e}}_{s j}^{m}(q)$ is the phonon mode displacement vector, and $\vec{\lambda}$ is the deformation potential vector.

In general, the deformation potential vector is the three-center integral

$$
\vec{\lambda}=\int \mathrm{d} \mathbf{r} \phi_{\pi}^{*}\left(\mathbf{r}-\mathbf{R}_{s^{\prime} j^{\prime}}^{l^{\prime}}\right) \nabla v\left(\mathbf{r}-\mathbf{R}_{s^{\prime \prime} j^{\prime \prime}}^{0}\right) \phi_{\pi}\left(\mathbf{r}-\mathbf{R}_{s j}^{l}\right) .
$$

We evaluate $\vec{\lambda}$ using the $2 p_{z}$ atomic wavefunctions $\phi_{\pi}(\mathbf{r})$ and screened atomic potential $v(\mathbf{r})$ for carbon in [113] obtained from an ab initio calculation in graphene [114].

The one-center deformation potential vector vanishes identically by symmetry and the dominant deformation potential vectors $\vec{\lambda}$ are the two-center integrals shown in figure 13 as functions of the interatomic distance. The distance $R$ between neighboring atoms is measured relative to the origin of the screened atomic potential $v(\mathbf{r})$. These integrals reach a maximum around $0.5 \AA$ and fall off sharply with distance. In figure 13, the equilibrium distances from a carbon atom to the first four nearest neighbor shells are indicated.

Further details concerning the evaluation of the electron-phonon matrix elements in SWNTs and GNRs can be found in the appendices of [54] and [47].

3.4.2. Effective mass theory. We can also calculate the RBM and RBLM electron-phonon matrix elements by using effective mass theory. Indeed, considering the effective mass theory allows us to analyze the trend of $\mathrm{CP}$ amplitudes of SWNTS and GNRs which will be discussed later. Here we briefly derive the Hamiltonian needed to calculate the electron-phonon matrix elements. The electron-phonon Hamiltonian in effective mass theory for graphene-related systems basically can be decomposed into the on-site and off-site Hamiltonians,

$$
\mathcal{H}^{(\mathrm{ep})}=\mathcal{H}_{\text {on }}+\mathcal{H}_{\text {off }}
$$

The details of the on-site and off-site interactions are given in Sasaki's work on the deformation-induced gauge field in graphene [59]. We will directly use his results in formulating the on-site and off-site Hamiltonians. The on-site and off-site interactions are induced by a lattice deformation which gives rise to a change in the transfer integral and a change in the potential between A and B atoms in the graphene unit cell. We adopt a coordinate system shown in figure 14 to derive $\mathcal{H}^{(\mathrm{ep})}$.

The on-site Hamiltonian can be expressed in terms of the divergence of $\mathbf{u}_{\mathrm{A}}$ and $\mathbf{u}_{\mathrm{B}}$, which represent the displacement vector of A-atom and B-atom in the graphene unit cell, respectively. This Hamiltonian is written as

$$
\mathcal{H}_{\text {on }}=g_{\text {on }}\left(\begin{array}{cc}
\nabla \cdot \mathbf{u}_{\mathrm{B}}(\mathbf{r}) & 0 \\
0 & \nabla \cdot \mathbf{u}_{\mathrm{A}}(\mathbf{r})
\end{array}\right) .
$$

For the discussion of the RBM and RBLM electron-phonon interactions, we rewrite (12) as follows:

$$
\begin{aligned}
\mathcal{H}_{\mathrm{on}}= & g_{\text {on }} \sigma_{0} \nabla \cdot\left(\frac{\mathbf{u}_{\mathrm{A}}(\mathbf{r})+\mathbf{u}_{\mathrm{B}}(\mathbf{r})}{2}\right) \\
& +g_{\text {on }} \sigma_{z} \nabla \cdot\left(\frac{\mathbf{u}_{\mathrm{A}}(\mathbf{r})-\mathbf{u}_{\mathrm{B}}(\mathbf{r})}{2}\right),
\end{aligned}
$$

where $g_{\text {on }}$ denotes the gradient of the atomic potential at $\mathbf{r}, \sigma_{0}$ is the identity matrix, and $\sigma_{z}$ is the $z$-component of the vector of Pauli matrices. In the simplest case, we have $\mathbf{u}_{\mathrm{A}}(\mathbf{r})=$ $\mathbf{u}_{\mathrm{B}}(\mathbf{r})=\mathbf{u}(\mathbf{r})$. Therefore, equation (13) can be simplified to be

$$
\mathcal{H}_{\text {on }}=g_{\text {on }} \sigma_{0} \nabla \cdot \mathbf{u}(\mathbf{r}) .
$$

Let $\nabla \cdot \mathbf{u}(\mathbf{r})=u_{\mathrm{ph}}$, we can write equation (14) as

$$
\mathcal{H}_{\mathrm{on}}=u_{\mathrm{ph}}\left(\begin{array}{cc}
g_{\text {on }} & 0 \\
0 & g_{\text {on }}
\end{array}\right)
$$

In equation (15), $u_{\mathrm{ph}}$ is just a parameter determined by the type of vibrations. For example, in the case of zigzag nanotube 

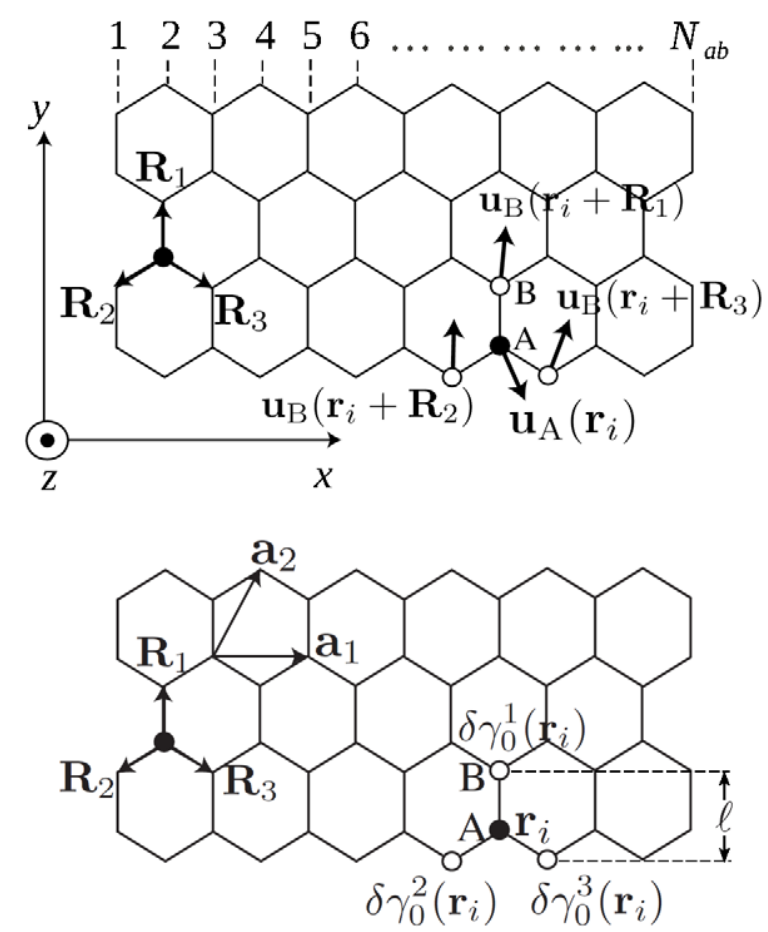

Figure 14. Upper panel shows displacements of B atoms at $\mathbf{r}_{i}+\mathbf{R}_{a}$ $(a=1,2,3)$, that is $\mathbf{u}_{\mathrm{B}}\left(\mathbf{r}_{i}+\mathbf{R}_{a}\right)$, which give rise to a deformation potential at A-atom of $\mathbf{r}_{i}$. Lower panel shows local modulations of the hopping integral defined by $\delta \gamma_{0}^{a}(\mathbf{r})(a=1,2,3)$. In this coordinate system we have the nearest neighbor vectors

$\mathbf{R}_{1}=\left(0, a_{\mathrm{cc}}\right), \mathbf{R}_{2}=(-\sqrt{3} / 2,-1 / 2) a_{\mathrm{cc}}, \mathbf{R}_{3}=(\sqrt{3} / 2,-1 / 2) a_{\mathrm{cc}}$, where $a_{\mathrm{cc}}=a / \sqrt{3}$. Here $\ell=3 a_{\mathrm{cc}} / 2$ is used in equation (19) (reproduced from [47]).

RBM oscillation, $u_{\mathrm{ph}}$ is found to be $2 s_{r} / d_{\mathrm{t}}$, where $s_{r}=$ $\sqrt{\hbar / 2 m_{\mathrm{c}} \omega_{\mathrm{RBM}}}$ is the phonon amplitude for the RBM [115] and $m_{\mathrm{c}}$ is total mass of carbon atoms in the unit cell.

Next, to derive the off-site interaction Hamiltonian, we start with the fact that the lattice deformation modifies the nearest neighbor hopping integral locally as $-\gamma_{0} \rightarrow-\gamma_{0}+$ $\delta \gamma_{0}^{a}\left(\mathbf{r}_{i}\right)(a=1,2,3)$. The corresponding perturbation of the lattice deformation is given by

$$
\mathcal{H}_{\text {deform }} \equiv \sum_{i \in \mathrm{A}} \sum_{a=1,2,3} \delta \gamma_{0}^{a}\left(\mathbf{r}_{i}\right)\left[\left(c_{i+a}^{\mathrm{B}}\right)^{\dagger} c_{i}^{\mathrm{A}}+\left(c_{i}^{\mathrm{A}}\right)^{\dagger} c_{i+a}^{\mathrm{B}}\right],
$$

where $c_{i}^{\mathrm{A}}$ is the annihilation operator for a $\pi$ electron on an A-atom at position $\mathbf{r}_{i}$, and $\left(c_{i+a}^{\mathrm{B}}\right)^{\dagger}$ is the creation operator for a $\pi$ electron on a B-atom at position $\mathbf{r}_{i+a}\left(=\mathbf{r}_{i}+\mathbf{R}_{a}\right)$. This perturbation gives rise to scattering within a region near the $\mathrm{K}$ point of graphene whose interaction is given by a deformation-induced gauge field $\mathbf{A}(\mathbf{r})=\left(A_{x}(\mathbf{r}), A_{y}(\mathbf{r})\right)$ as $v_{\mathrm{F}} \boldsymbol{\sigma} \cdot[\hat{\mathbf{p}}+\mathbf{A}(\mathbf{r})]$, where $v_{\mathrm{F}}$ is the Fermi velocity, $\hat{\mathbf{p}}=$ $-\mathrm{i} \hbar \nabla$ is the momentum operator, and $\boldsymbol{\sigma}$ is the Pauli matrix. The deformation-induced gauge field $\mathbf{A}(\mathbf{r})$ for the off-site interaction is defined from $\delta \gamma_{0}^{a}(\mathbf{r})(a=1,2,3)$ as

$$
\begin{aligned}
& v_{\mathrm{F}} A_{x}(\mathbf{r})=\delta \gamma_{0}^{1}(\mathbf{r})-\frac{1}{2}\left[\delta \gamma_{0}^{2}(\mathbf{r})+\delta \gamma_{0}^{3}(\mathbf{r})\right], \\
& v_{\mathrm{F}} A_{y}(\mathbf{r})=\frac{\sqrt{3}}{2}\left[\delta \gamma_{0}^{2}(\mathbf{r})-\delta \gamma_{0}^{3}(\mathbf{r})\right] .
\end{aligned}
$$

The perturbation to the nearest neighbor hopping integral for the RBM and RBLM electron-phonon interactions is given by

$$
\delta \gamma_{0}^{a}(\mathbf{r})=\frac{g_{\mathrm{off}}}{\ell a_{\mathrm{cc}}} \mathbf{R}_{a} \cdot\left\{\mathbf{u}\left(\mathbf{r}+\mathbf{R}_{a}\right)-\mathbf{u}(\mathbf{r})\right\}
$$

where $g_{\text {off }}$ is the off-site coupling constant and $\ell=3 a_{\mathrm{cc}} / 2$ (see the lower panel of figure 14). Here the displacement vector of a carbon atom at $\mathbf{r}$ in general is expressed by $\mathbf{u}(\mathbf{r})=$ $\left[u_{x}(\mathbf{r}), u_{y}(\mathbf{r})\right]$. Using a Taylor expansion, we approximate equation (19) as

$$
\delta \gamma_{0}^{a}(\mathbf{r})=\frac{g_{\text {off }}}{\ell a_{\mathrm{cc}}} \mathbf{R}_{a} \cdot\left\{\left(\mathbf{R}_{a} \cdot \nabla\right) \mathbf{u}(\mathbf{r})\right\} .
$$

Using $\mathbf{R}_{1}, \mathbf{R}_{2}$, and $\mathbf{R}_{3}$ in figure 14 , we obtain the deformation-induced gauge field of equations (17) and (18) as follows:

$$
\begin{aligned}
& v_{\mathrm{F}} A_{x}(\mathbf{r})=\frac{g_{\text {off }}}{2}\left[-\frac{\partial u_{x}(\mathbf{r})}{\partial x}+\frac{\partial u_{y}(\mathbf{r})}{\partial y}\right], \\
& v_{\mathrm{F}} A_{y}(\mathbf{r})=\frac{g_{\text {off }}}{2}\left[\frac{\partial u_{x}(\mathbf{r})}{\partial y}+\frac{\partial u_{y}(\mathbf{r})}{\partial x}\right] .
\end{aligned}
$$

Interestingly, $v_{\mathrm{F}} A_{y}=0$ for both RBM and RBLM cases [47, 115]. Therefore, the off-site Hamiltonian can be written as

$$
\mathcal{H}_{\mathrm{off}}=\sigma_{x} \nu_{\mathrm{F}} A_{x}=u_{\mathrm{ph}}\left(\begin{array}{cc}
0 & -\frac{g_{\text {off }}}{2} \\
-\frac{g_{\text {off }}}{2} & 0
\end{array}\right) .
$$

\subsection{Generation of coherent phonons}

The coherent phonon amplitude is related to the expectation value of the phonon displacement operator. If we take the expectation value of the atomic displacement operator $\mathbf{U}_{s j}^{l}(t) \equiv\left\langle\mathbf{U}_{s j}^{l}\right\rangle$ we get an expression for the time-dependent atomic displacements

$$
\mathbf{U}_{s j}^{l}(t)=\frac{\hbar}{\sqrt{2 \rho L_{\Omega}}} \sum_{m q} \frac{\hat{\mathbf{e}}_{s j}^{m}(q)}{\sqrt{\hbar \omega_{m}(q)}} \mathrm{e}^{\mathrm{i} q T l} Q_{m q}(t)
$$

where $Q_{m q}(t)=\left\langle b_{m q}+b_{m,-q}^{\dagger}\right\rangle$ is the coherent phonon amplitude.

In $\mathrm{CP}$ spectroscopy, the coherent phonon modes that are typically excited are the ones with wavevector $q=0$. From the second-quantized form of the free phonon and deformation potential electron-phonon Hamiltonians, we can use the Heisenberg equation to motion to show that the CP amplitudes $Q_{m}(t) \equiv Q_{m, q=0}(t)$ satisfy a driven oscillator equation $[47,54,70]$

$$
\frac{\partial^{2} Q_{m}(t)}{\partial t^{2}}+\omega_{m}^{2} Q_{m}(t)=S_{m}(t),
$$

where $m$ denotes the phonon mode and $\omega_{m}$ is the frequency of phonon mode $m$ at $q=0$. In the ETB model, the coherent lattice displacements can then be expressed in terms of the $\mathrm{CP}$ amplitudes $Q_{m}(t)$. Explicit expressions for individual carbon atom displacements as functions of the $\mathrm{CP}$ amplitudes in SWNTs and GNRs are given in [54] and [47] respectively. 
There is no damping term in equation (25) since anharmonic terms in the electron-phonon Hamiltonian are neglected. We solve the driven oscillator equation subject to the initial conditions $Q_{m}(0)=0$ and $\dot{Q}_{m}(0)=0$. The driving function $S_{m}(t)$ is given by

$$
S_{m}(t)=-\frac{2 \omega_{m}}{\hbar} \sum_{n k} M_{n}^{m}(k)\left(f_{n}(k, t)-f_{n}^{0}(k)\right)
$$

where $f_{n}^{0}(k)$ and $f_{n}(k, t)$ are the initial and time-dependent electron distribution functions, respectively. Here $n$ labels the electronic state and $k$ is the electron wavevector. The $q=$ 0 deformation potential electron-phonon interaction matrix element in our ETB model is $M_{n}^{m}(k) \equiv M_{n k ; n k}^{m, q=0}[47,54]$.

When we neglect slow carrier relaxation effects and retain only the photogeneration term in the Boltzmann equation (impulsive excitation approximation), the net photogenerated conduction band electron distribution function $f_{\mathrm{c}}(k, t)-f_{\mathrm{c}}^{0}(k)$ for any optical transition from the valence band to the conduction band ( $\mathrm{v} \rightarrow \mathrm{c}$ ) is equal to the net photogenerated hole distribution function $f_{\mathrm{v}}^{0}(k)-f_{\mathrm{v}}(k, t)$ for each value of $k$. In this case we obtain a simplified expression for the driving function in terms of the conduction band distribution functions. Thus

$$
S_{m}(t)=\sum_{\mathrm{c} k} S_{m}(k)\left(f_{\mathrm{c}}(k, t)-f_{\mathrm{c}}^{0}(k)\right),
$$

where the driving function kernel $S_{m}(k)$ for the $(\mathrm{v} \rightarrow \mathrm{c})$ transition is

$$
S_{m}(k)=-\frac{2 \omega_{m}}{\hbar}\left(M_{\mathrm{c}}^{m}(k)-M_{\mathrm{v}}^{m}(k)\right) .
$$

The driving function kernels in equation (27) for generation of coherent LO and TO phonons in graphene have been studied by Sanders $e t$ al in [116] for the $\pi$ band $(\mathrm{v} \rightarrow \mathrm{c})$ transition and are shown in figure 15 as a function of $k$. As a guide to the eyes, the $S=-10^{6} \mathrm{ps}^{-2}$ contour is shown as a dotted (yellow) line and the $S=+10^{6} \mathrm{ps}^{-2}$ contour appears as a solid (red) line. We note that $S_{m}(k)$ is even under reflection about the $k_{x}$ and $k_{y}$ axes for coherent LO phonons while $S_{m}(k)$ for coherent TO phonons is odd under reflections about the $k_{x}$ and $k_{y}$ axes.

\subsection{Detection of coherent phonons}

In coherent phonon spectroscopy a probe pulse is used to measure the time-varying absorption coefficient $\alpha(\hbar \omega, t)$ at the probe energy. The time-varying absorption coefficient is computed using Fermi's golden rule and in the golden rule expression for the absorption coefficient, we explicitly take the time variation of the band structure and carrier distribution functions into account. The time dependence of the band structure comes from changes induced by the coherent phonon lattice displacements which alter bond lengths between carbon atoms. The bond length variations depend on the coherent phonon modes being excited and their phases. We make the assumption that the electrons adiabatically follow the lattice vibrations and use the ETB model to calculate changes in the electronic structure. From the computed differential transmission at the probe energy, we

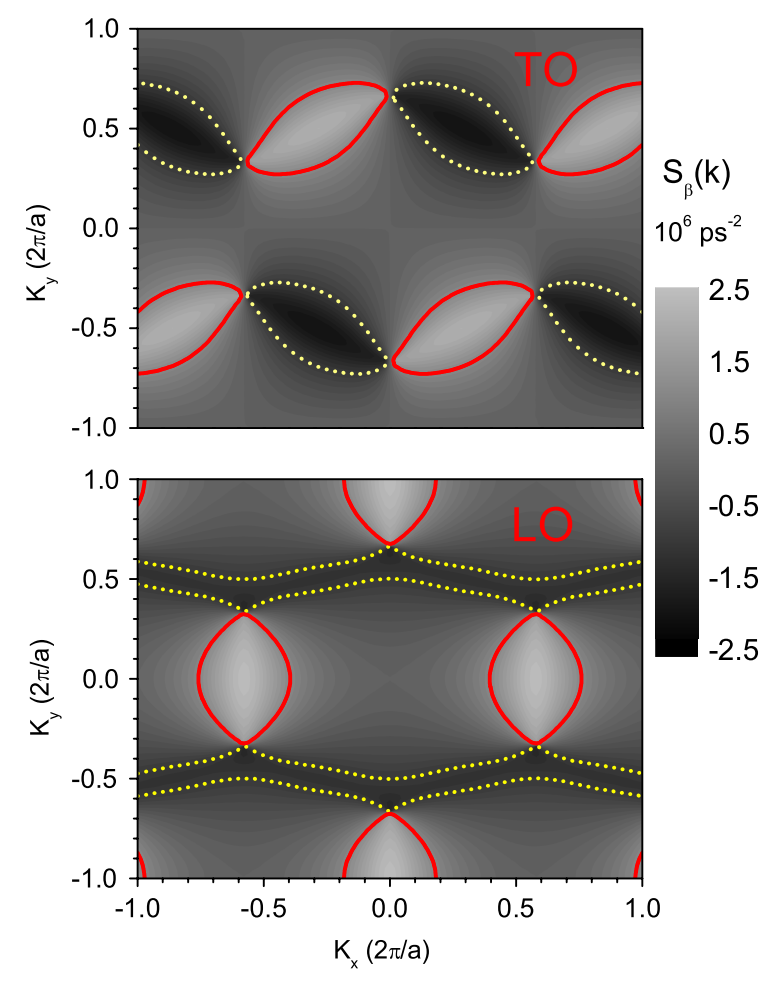

Figure 15. Driving function kernel $S_{m}(\mathbf{k})$ for coherent LO and TO phonons for graphene in the impulsive excitation approximation. The dotted (yellow) and solid (red) contour lines correspond to $S=-10^{6}$ and $+10^{6} \mathrm{ps}^{-2}$ respectively (reproduced from [116]).

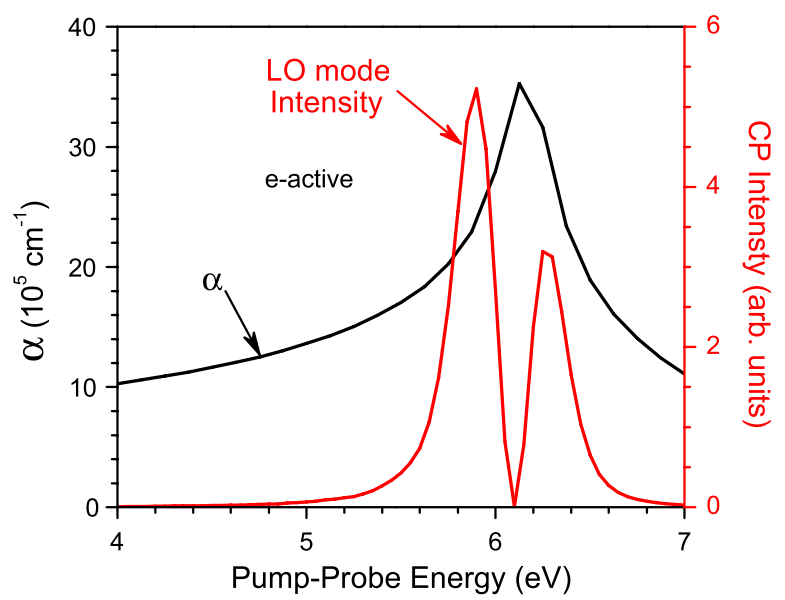

Figure 16. Graphene absorption coefficient as a function of pump excitation energy for normally incident e-actively polarized light plotted as a solid black line against the left axis. The CP intensity as a function of excitation energy is plotted as a solid red line against right axis (reproduced from [116]).

obtain the $\mathrm{CP}$ intensity by taking the Fourier transform of the differential transmission.

An example of such a calculation in graphene is shown in figure 16 where we plot the graphene absorption coefficient as a function of excitation energy for e-actively polarized pump/probe beams. The photon excitation energy is in the vicinity of the $M$ point transition in graphene and the $C P$ intensity induced by the coherent LO mode is seen to be 


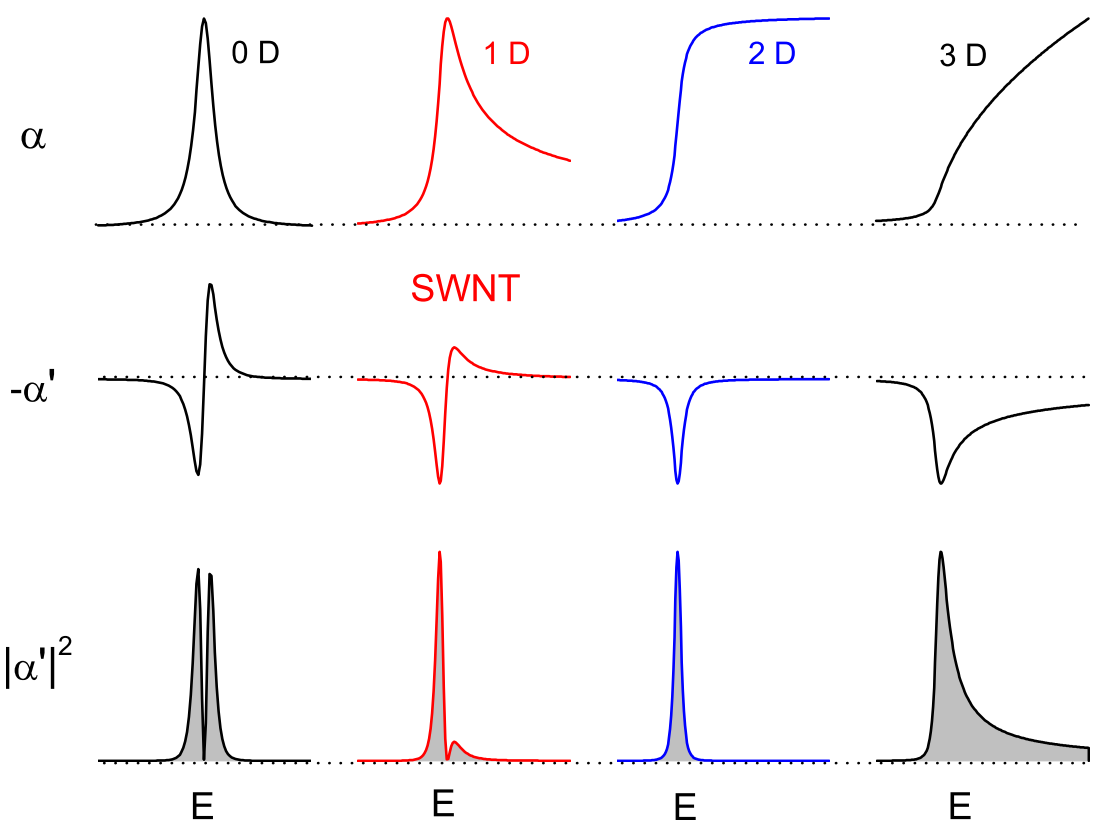

Figure 17. Schematic dimensionality dependence of CP signal. Top row: absorption coefficient versus photon energy for $0,1,2$, and 3-dimensional carbon structures. Middle row: negative of the derivative of the absorption coefficient proportional to transient differential transmission. Bottom row: square of the derivative of the absorption coefficient proportional to the $\mathrm{CP}$ intensity.

roughly proportional to the square of the derivative of the absorption coefficient. Thus, CP spectroscopy is a derivative spectroscopy.

This is a generic feature and can be qualitatively understood for photoexcitation near the band edge. For coherent RBM oscillations in semiconducting SWNTs, the coherent tube diameter oscillations give rise to band gap oscillations which, to first approximation, rigidly shifts the absorption spectrum. In coherent phonon spectroscopy on semiconducting carbon structures, excitation of coherent phonons near the band edge by the pump modulates the band gap and gives rise to transient differential transmission. We take the theoretical CP signal to be proportional to the power spectrum of the transient differential transmission after background subtraction. Thus, the CP signal as a function of the pump energy is roughly proportional to the square of the derivative of the absorption coefficient. The dimensionality dependence of CP intensity in different carbon nanostructures is shown schematically in figure 17. The top row shows the absorption coefficient as a function of photon energy for carbon structures in $0,1,2$, and 3 dimensions. The middle row shows the negative of the derivative of the absorption coefficient which is proportional to the transient differential transmission due to $\mathrm{CP}$ induced band gap oscillations. The bottom row shows the square of the derivative of the absorption coefficient which is roughly proportional to the $\mathrm{CP}$ intensity.

In our ETB model, we explicitly track how the energy bands $E_{n}(k)$ are modified by coherent phonon oscillations. These shifts are taken as inputs to the Fermi golden rule calculation of the time-dependent optical properties measured by the probe beam. The energy band shifts depend on the band index $n$, the wavevector $k$, as well as the coherent

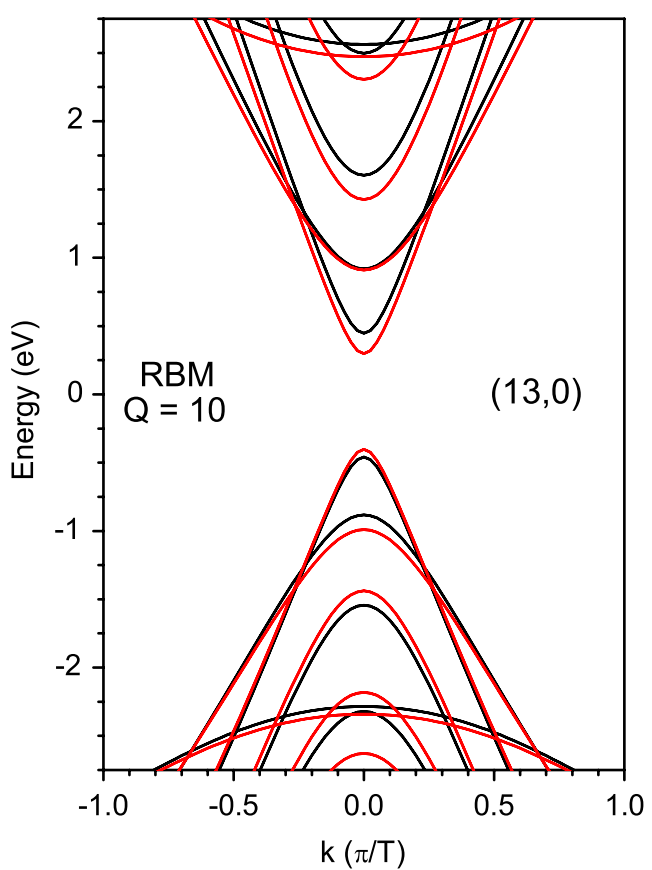

Figure 18. Effect of coherent RBM phonon oscillations on SWNT bands for $(13,0)$ SWNTs. Black curves are unstrained bands and red curves are adiabatically modified bands due to RBM oscillations when the RBM coherent $\mathrm{CP}$ amplitude is set to a large value of $Q=10$.

phonon mode being excited. For the RBM coherent phonon mode, figure 18 shows how the bands in $(13,0)$ SWNTs are modified by the coherent RBM phonon oscillations. The black curves are the unmodified bands and the red curves are bands calculated when the RBM coherent phonon amplitude is fixed 

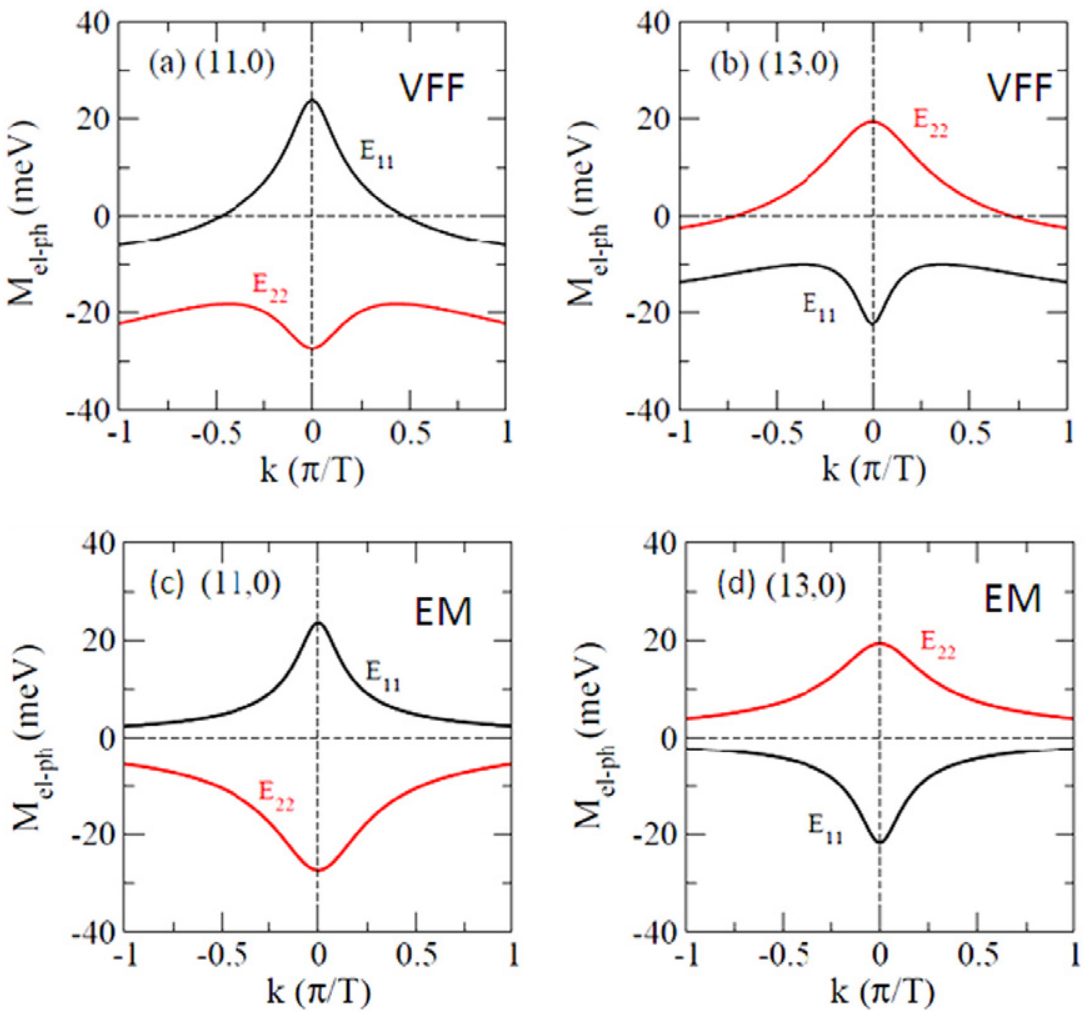

Figure 19. Electron-phonon matrix elements for coherent RBM phonons in (a) $(11,0)$ and (b) $(13,0)$ SWNTs for $E_{11}$ (black curve) and $E_{22}$ (red curve) optical transitions calculated within the ETB formalism with VFF phonon modes. Electron-phonon matrix elements calculated using effective mass theory for (c) $(11,0)$ and (d) $(13,0)$ SWNTs. The CP driving function $S(k)$ is proportional to the negative of the electron-phonon matrix element and the sign convention is chosen so that a positive electron-phonon matrix element corresponds to a radially inward driving term $S(k)$ for RBM coherent phonons.

at $Q=10$. In actual RBM oscillations, $Q \ll 10$ so the band gap shifts shown in the figure are greatly exaggerated. The size of the band gap shift varies slowly with $k$, so the shifts may be taken as approximately rigid. However, the magnitude and sign of the shifts vary depending on the optical transition. We note that for $Q>0$ the $E_{11}$ band gap shrinks at $k=0$ while the $E_{22}$ band gap expands.

\section{Coherent phonons in carbon nanotubes}

Using the concepts we have developed in section 3, we are now able to investigate coherent phonon properties in a variety of SWNT systems. We first investigate how the initial phase of the CP amplitude depends upon tube type and chirality. Then we compare our calculations with two experimental SWNT systems: (1) micelle suspended nanotubes and (2) aligned films of nanotubes. Comparison of theory with experiment is difficult because usually there are an ensemble of randomly oriented different types and chiralities of the SWNTs in a given sample, making it difficult to compare to theory. However, in the first type of experiment on micelle suspended tubes, one can use femtosecond pulse shaping to resonantly excite CPs in a tube of a fixed chirality. In the second type of experiment, the ability to grow films of aligned nanotubes (with approximately the same diameter but different chiralities) allows one to study the dependence of the $\mathrm{CP}$ signal on the polarization of the pump and probe with respect to the nanotube axis.

\subsection{Initial phase of the RBM CP amplitude}

The lowest lying CP mode is the $q=0 \mathrm{RBM}$ mode in which the tube diameter oscillates about an equilibrium value. In this case, the $\mathrm{CP}$ amplitude $Q(t)$ is directly proportional to the tube diameter $D(t)$ [54]. The driving function kernels are defined on the one-dimensional nanotube Brillouin zone and there is a different kernel for each optical transition $E_{i i}$. For light polarized along the nanotube growth direction, the allowed transitions are between conduction and valence bands on the same cutting line and thus various optical transitions can be associated with the cutting lines.

The RBM electron-phonon matrix elements in the ETB model for $E_{11}$ and $E_{22}$ transitions in semiconducting (type-I) $(11,0)$ and (type-II) $(13,0)$ nanotubes are shown in figures 19(a) and (b), respectively. Here type-I or II nanotube families are determined by $\bmod (2 n+m, 3)=$ 1 or 2 , respectively. $\operatorname{Mod}(2 n+m, 3)=0$ corresponds to a metallic SWNT. Electron-phonon matrix elements calculated in the effective mass theory and shown in figures $19(\mathrm{c})$ and (d), respectively (while $\bmod (2 n+m, 3)=0$ corresponds to a metallic SWNT). Comparing figures 19(a) and (c) and comparing figures 19(b) and (d) we see that there is good agreement between ETB and effective 
mass results in the region around $k=0$. The RBM coherent phonon driving function $S(k)$ is proportional to the negative of the electron-phonon matrix element so a positive electron-phonon matrix element gives a negative contribution to $S(k)$. Comparing figures 19(a) and (b) we see that photoexcitation at the $E_{22}$ band edge causes the coherent RBM phonon to initially expand in the case of the $(11,0)$ nanotube and contract in the case of the $(13,0)$ nanotube. The opposite is true for photoexcitation at the $E_{11}$ transition. When photoexcited by ultrafast laser pulses, lattices in solids tend to expand in accordance with the Franck-Condon principle. As seen in figure 19 this is not the case for coherent RBM oscillations in carbon nanotubes where the diameter can initially expand or contract depending on nanotube chirality and photoexcitation energy. To better understand this phenomenon, we undertook a systematic study of the chirality dependence of coherent RBM oscillation amplitudes within the effective mass framework by considering the RBM electron-phonon interaction analytically [74].

In a nearest neighbor effective mass approximation, the RBM electron-phonon matrix element $\mathcal{H}^{(\mathrm{ep})}$ for an $(n, m)$ SWNT with a chiral angle $\theta$ and diameter $d_{\mathrm{t}}$ is obtained from a sum of the on-site and off-site electron-phonon Hamiltonians in equations (15) and (23), respectively. However, we should add a phase factor $\mathrm{e}^{\mathrm{i} 3 \theta}$ to the off-site Hamiltonian in order to take into account a general chirality dependence. Therefore, the RBM electron-phonon matrix element can be written as

$$
\mathcal{H}^{(\mathrm{ep})}=\frac{2 s_{r}}{d_{\mathrm{t}}}\left(\begin{array}{cc}
g_{\text {on }} & -\frac{g_{\text {off }}}{2} \mathrm{e}^{\mathrm{i} 3 \theta} \\
-\frac{g_{\text {off }}}{2} \mathrm{e}^{-\mathrm{i} 3 \theta} & g_{\text {on }}
\end{array}\right),
$$

where $g_{\text {on }}\left(g_{\text {off }}\right)$ is the on-site (off-site) coupling constant. Here $s_{r}=\sqrt{\hbar / 2 m_{\mathrm{c}} \omega_{\mathrm{RBM}}}$ is the phonon amplitude for the $\mathrm{RBM}, \omega_{\mathrm{RBM}}$ is the RBM phonon frequency at $q=0$, and $m_{\mathrm{c}}$ is the total mass of the carbon atoms within the unit cell. The electron-phonon matrix element is defined as a sum of conduction band $c$ and valence band $v$ electron-phonon matrix elements [117], which represent the electron and hole contributions, respectively,

$$
M^{(\mathrm{ep})}=M_{\mathrm{c}}^{(\mathrm{ep})}-M_{\mathrm{v}}^{(\mathrm{ep})}=\left\langle c\left|\mathcal{H}^{(\mathrm{ep})}\right| c\right\rangle-\left\langle v\left|\mathcal{H}^{(\mathrm{ep})}\right| v\right\rangle .
$$

To obtain $M^{(\mathrm{ep})}$ in (30), we use the following wavefunctions,

$$
\begin{aligned}
\Psi_{\mathrm{c}} & =\frac{\mathrm{e}^{\mathrm{i} \boldsymbol{k} \cdot \boldsymbol{r}}}{\sqrt{2 S}}\left(\begin{array}{c}
\mathrm{e}^{-\mathrm{i} \Theta(\boldsymbol{k}) / 2} \\
\mathrm{e}^{+\mathrm{i} \Theta(\boldsymbol{k}) / 2}
\end{array}\right), \\
\Psi_{\mathrm{v}} & =\frac{\mathrm{e}^{\mathrm{i} \boldsymbol{k} \cdot \boldsymbol{r}}}{\sqrt{2 S}}\left(\begin{array}{c}
\mathrm{e}^{-\mathrm{i} \Theta(\boldsymbol{k}) / 2} \\
-\mathrm{e}^{+\mathrm{i} \Theta(\boldsymbol{k}) / 2}
\end{array}\right),
\end{aligned}
$$

for conduction and valence states, respectively, which are suitable near the graphene K point [115]. In (31), $S$ is the surface area of graphene and $\Theta(\boldsymbol{k})$ is an angle at the $\mathrm{K}$ point measured from a line perpendicular to the cutting lines (figure 20). By inserting the wavefunctions in (31) to (30), we obtain

$$
M^{(\mathrm{ep})}=-\frac{s_{r}}{d_{\mathrm{t}}}\left[g_{\text {off }} \cos (\Theta(\boldsymbol{k})+3 \theta)\right]
$$
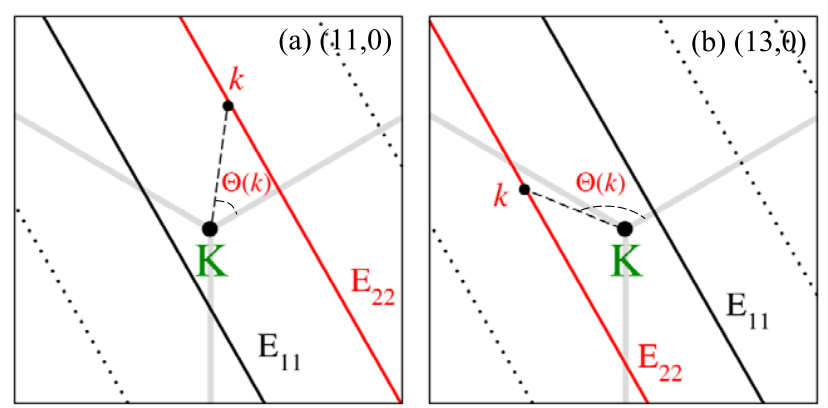

Figure 20. Cutting lines for (a) $(11,0)$ and (b) $(13,0)$ zigzag nanotubes near the graphene K point. Solid lines denote the $E_{11}$ and $E_{22}$ cutting lines, respectively, while the dotted lines correspond to higher cutting lines. The angle $\Theta(k)$ defines the position of a $k$ point. Here $\Theta(k)$ is shown for a $k$ point on the $E_{22}$ cutting line for both SWNTs. The difference between the type-I and type-II families can be understood from the position of the $E_{11}$ or $E_{22}$ cutting lines relative to the $\mathrm{K}$ point (reproduced from [74]).

Table 1. Initial lattice behavior due to coherent RBM oscillations at $E_{11}$ and $E_{22}$ in semiconducting SWNTs.

\begin{tabular}{lll}
\hline Nanotube family & $E_{11}$ & $E_{22}$ \\
\hline Type I $(\bmod (2 n+m, 3)=1)$ & Contract & Expand \\
Type II $(\bmod (2 n+m, 3)=2)$ & Expand & Contract \\
\hline
\end{tabular}

According to the density-functional calculation by Porezag et al [82], we may adopt the off-site coupling constant $g_{\text {off }}=6.4 \mathrm{eV}$ and the on-site coupling constant $g_{\text {on }}=$ $17.0 \mathrm{eV}$, which are calculated for the first nearest neighbor carbon-carbon distance [115]. However, $g_{\text {on }}$ has no effect on the electron-phonon matrix element since it vanishes in (32). Finally, using the matrix element (32), the coherent RBM driving function kernel in (28) is then given by

$$
S(\mathbf{k})=\frac{2 \omega_{\mathrm{RBM}}}{\hbar} \frac{s_{r}}{d_{\mathrm{t}}}\left[2 g_{\text {off }} \cos (\Theta(\mathbf{k})+3 \theta)\right] .
$$

In the cutting line picture of figure 20, the angle $\Theta(\boldsymbol{k})$ is measured counterclockwise from a line perpendicular to the cutting lines, with the positive direction of the line to the right of the $\mathrm{K}$ point. A simple geometrical argument shows that the driving function (33) correctly predicts the sign of the results shown in figure 19 for the $E_{11}$ and $E_{22}$ transitions [74] based on the rule of $\Theta(\mathbf{k})$. For example, in figure 20 we show the cutting lines for the $(11,0)$ and $(13,0)$ nanotubes. The $E_{22}$ cutting line for the $(11,0)((13,0))$ tube is to the right (left) of the $\mathrm{K}$ point, giving a positive (negative) $\cos (\Theta(\boldsymbol{k}))$ and thus a positive (negative) $S(\mathbf{k})$ for the $E_{22}$ transition, corresponding to initial increase (decrease) of the tube diameter. The coherent RBM oscillations are thus strongly nanotube family dependent. Their behavior are summarized in table 1 . However, it is important to note that the general trend might deviate from table 1 because of the chiral angle $\theta$ term in (33). Also, for higher lying transitions with cutting lines far from the $\mathrm{K}$ point, the effective mass picture breaks down due the limitation of the first nearest neighbor approximation. 


\subsection{CP in micelle suspended SWNTs}

The electronic properties of carbon nanotubes depend on their chirality $(n, m)$ [7, 34, 76-78]. Carbon nanotube samples typically contain ensembles of nanotubes with different chiralities. The relative abundances of different chirality tubes makes it difficult to study chirality-dependent properties. Kim et al then developed resonant CP spectroscopy [50, 54], a technique that allows chirality-dependent properties of nanotubes in an ensemble to be studied. Using pre-designed trains of femtosecond optical pulses, it is possible to selectively excite and probe coherent RBM lattice vibrations in SWNTs of a specific chirality and gain information on light absorption, coherent phonon generation, and coherent phonon-induced band structure modulations. Coherent RBM phonons are selectively excited using a train of pump pulses whose repetition rate is equal to the desired phonon frequency. By exciting coherent phonons with a specific frequency, nanotubes with a single chirality in an ensemble of tubes can be studied.

Lim et al generated and detected RBM phonons in single-walled carbon nanotubes using CP spectroscopy with ultrafast pump pulses with durations of around $50 \mathrm{fs}[52,53]$. The coherent RBM phonons give rise to diameter oscillations. The band gap is roughly inversely related to the diameter, so the induced RBM diameter oscillations give rise to band gap oscillations at the phonon frequencies. These band gap oscillations induce oscillations in the excitonic peaks which are detected by the probe beam.

The CP spectra in SWNT ensemble samples exhibited a large number of strong peaks, each one induced by RBM diameter oscillations in nanotubes of different chiralities. The chiralities corresponding to the different peaks were identified from the RBM oscillation frequencies and the relative strengths of the peaks provides information on the relative populations of different chirality nanotubes in the ensemble samples.

With 50 fs laser pulses, coherent RBM phonon modes are excited since the pulse duration is much less than the RBM oscillation period around 100-200 fs. On the other hand, the coherent $\mathrm{G}$ mode phonon energies are near $0.2 \mathrm{eV}$ (see figure 10) with periods of around $21 \mathrm{fs}$. In order to excite coherent $\mathrm{G}$ mode phonons, it is necessary to use laser pulses shorter than 21 fs. Gambetta et al used sub-10-fs pump pulses to excite both coherent RBM and G modes in SWNT ensembles [57]. The anharmonic coupling between the RBM and $G$ modes resulted in frequency modulation of the $G$ modes by the RBM. Using quantum chemical dynamic simulations, they concluded that the nanotube surface becomes locally distorted during photoexcitation. This corrugation of the surface of the SWNT during photoexcitation couples the radial and longitudinal modes and gives rise to the observed frequency modulation of the G modes by the RBM [57].

In the above studies, coherent phonons were observed in semiconducting SWNTs but were not observed in metallic SWNTs. Using sub-10 fs laser pulses, Kato et al investigated coherent phonon dynamics in metallic SWNTs using CP spectroscopy experiments measuring time-resolved

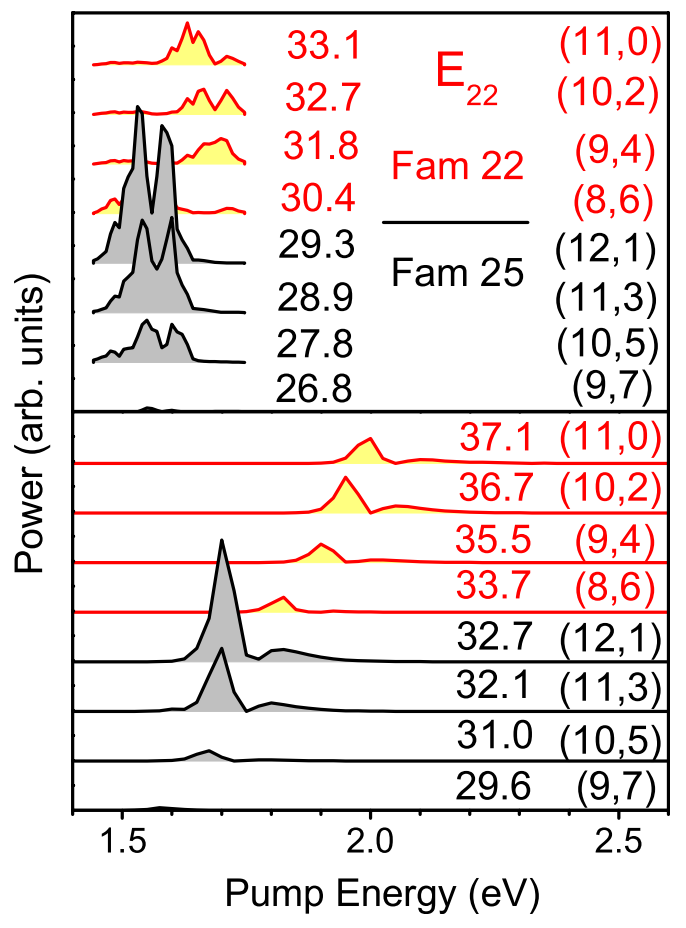

Figure 21. Coherent phonon intensity at the RBM frequency as a function of pump-probe energy for several mod 2 semiconducting nanotubes at the $E_{22}$ transition. The experimental CP spectra are in the top panel and the simulated CP spectra are in the bottom panel. The upper four curves in each panel are for nanotubes in Family 22 and the lower four curves are for tubes in Family 25. Each curve is labeled with the chirality $(n, m)$ and the RBM phonon energy in meV (adapted from [50]).

reflectivity with the probe pulse and observed coherent RBM, D mode, and $\mathrm{G}$ mode phonons [118]. In the case of coherent $\mathrm{G}$ mode phonons, they were able to resolve the longitudinal $\mathrm{G}^{-}$and transverse $\mathrm{G}^{+}$modes. The frequencies of the coherent RBM phonons were observed to shift with increasing pump intensity implying that the photocarriers modify the RBM phonon frequency.

In [54], Sanders et al used resonant CP spectroscopy to systematically study $\mathrm{CP}$ spectra in micelle suspended mod 2 semiconducting nanotubes of different chiralities and compared experimental and theoretical $\mathrm{CP}$ intensities at the RBM phonon frequency as a function of the excitation energy. As an aside on nomenclature, mod 2 semiconducting SWNTs with chirality indices $(n, m)$ satisfy $\bmod (n-m, 3)=2$ and are equivalent to semiconducting type I SWNTs which satisfy $\bmod (2 n+m, 3)=1$.

The results of the comparison of theory and experiment for mod 2 SWNTs are shown in figure 21. The bottom panel of figure 21 shows the theoretical CP intensity at the RBM frequency as a function of excitation energy for all $(n, m)$ nanotubes in Families with $2 n+m=22$ and 25. The curves for each nanotube are labeled with the nanotube chirality $(n, m)$ and the computed RBM phonon energy in meV. In each tube, peaks in the CP spectra correspond to $E_{22}$ transitions. Within each family, CP intensity tends to decrease as the chiral angle and tends to increase as we go from Family 22 to Family 25. The top panel of figure 21 shows the corresponding 


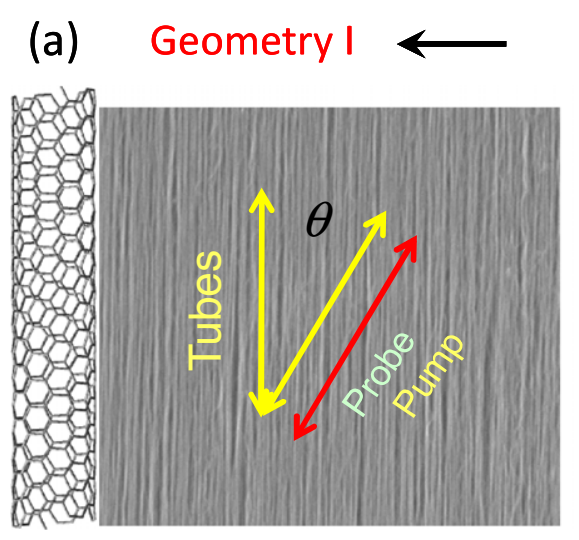

(b) $\longrightarrow$ Geometry II

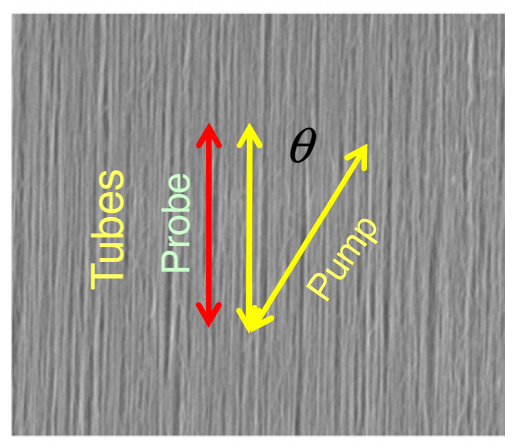

Figure 22. Schematic diagrams showing experimental configurations employed in polarization-dependent pump-probe CP measurements reported by Booshehri et al in [56]. In (a) the pump and probe polarizations are fixed and the sample is rotated through an angle $\theta$. In (b) the probe and sample orientations are fixed and the pump polarization is rotated through an angle $\phi$.

experimental CP spectra for tubes in Families 22 and 25. We see that the theory correctly predicts overall trends in the $\mathrm{CP}$ intensities both within and between Families. There is a small difference in the calculated RBM energy compared to the experimental energy with the experimental RBM energy $\approx 3-4 \mathrm{meV}$ lower. We attribute this difference to the fact that the experimental tubes are micelle suspended in liquid and we would expect them to have a lower RBM frequency than an isolated tube.

Discrepancies in the predicted excitation energies of the peaks on the order of $0.4 \mathrm{eV}$ or less are observed. This discrepancy is most likely due to Coulomb interactions (or excitonic effects) which are not included in the present theory. Both the excitonic red shift and the self-energy blue shift are on the order of $\mathrm{eV}$ in nanotubes, with the latter exceeding the former [37, 91, 92, 119-121]. The dielectric function of the surrounding medium in the micelle suspended samples also influences excitonic transition energies [122]. Comparing theoretical and experimental CP spectra for the $(12,1)$ tube, we find that both exhibit a double peaked structure, but the lower energy theoretical peak is much stronger than the higher energy peak whereas the two experimental peaks have comparable strength. This discrepancy is due to excitonic modification of the shape of the nanotube absorption spectrum whose time-dependent modulation gives rise to the shape of the CP signal [52]. This is illustrated again in figure 17. There we see that the expected CP lineshape for a OD system (as one would expect for strong exciton effects) more closely matches the experimental lineshape than the 1D system.

\subsection{Polarization dependence of the CP signal in aligned nanotube films}

Kim et al [123] and Kato et al [118] studied the polarization dependence of CP oscillations in SWNTs and the relation between the nanotube axis and the incident light polarization. They found that optical absorption in nanotubes depends strongly on polarization. CP amplitudes measured as a function of the angle between the pump and probe polarizations were analyzed based on the absorption anisotropy of carbon nanotubes. A simple model in which nanotubes in a solution sample have random orientations and taking the expected optical anisotropy of nanotubes into account was used to explain observed polarization-dependent $\mathrm{CP}$ oscillations as a function of the angle $\theta$ between the pump and probe beams. It is found that the $\mathrm{CP}$ intensity can be fitted to $I(\theta)=A\left(2 \cos ^{2}(\theta)+1\right)+B$ where $A$ and $B$ are fitting parameters. This agrees well with experiment with $A=0.234$ and $B=0.302$ as seen in figure 5 of [123].

A more elaborate study of polarization dependence of coherent phonons in samples where the tubes are highly aligned was undertaken by Booshehri et al [56]. These authors used polarization-dependent differential-transmission pump-probe CP spectroscopy to investigate the polarization dependence of coherent RBM phonons in highly aligned single-walled carbon nanotube thin films transferred onto a sapphire substrate with a diameter distributed between 1 and $5 \mathrm{~nm}$ centered around $3 \mathrm{~nm}$ [124]. Experiments on RBM CPs were performed in two different geometries indicated schematically in figure 22. In geometry I (figure 22(a)), the pump and probe polarizations are fixed, and the sample orientation is rotated through an angle $\theta$. In geometry II (figure 22(b)), the probe polarization and sample orientations are fixed, and the pump polarization rotated through an angle $\phi$.

The optical properties of carbon nanotubes are very anisotropic with the strongest RBM CP signal occurring when the pump polarization is parallel to the tube axis. It was found that very nearly complete quenching of the RBM CPs was observed in both experimental geometries when the pump polarization was perpendicular to the tube axis. Comparing experimental results with theory, Booshehri et al simulated polarization-dependent $\mathrm{CP}$ spectroscopy in a $(38,0)$ zigzag nanotube and found a decrease in CP signal as the optical polarization varied from parallel to perpendicular to the tube axis [56]. Using those simulated results, they found a

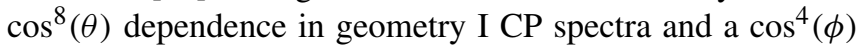
polarization dependence in geometry II CP spectra. In fitting experiment to theory, misalignment effects were taken into account, and it was determined that data from both geometry I and II experiments could be fit assuming the nematic order 


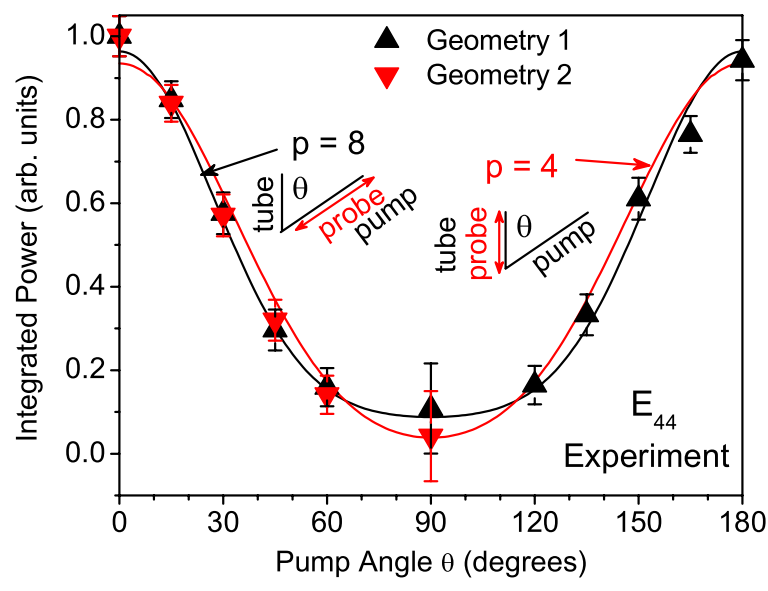

Figure 23. Experimental integrated coherent RBM phonon power for the $E_{44}$ transition as a function of $\theta$ for Geometry 1 (upward pointing triangles) and $\phi$ for Geometry 2 (downward pointing triangles) experiments. Geometry 1 experiments are fit to $A \cos ^{p}(\theta+\Delta \theta)+B$ where $p=8$ (dashed line) and Geometry 2 experiments are fit to $A \cos ^{p}(\phi+\Delta \phi)+B$ for $p=4$ (solid red line) $A$ and $B$ are background subtraction and rescaling parameters and the standard deviations for the random tube axis misalignment $\Delta \theta$ and $\Delta \phi$ are restricted to be the same for both Geometry 1 and 2 experiments (reproduced from [56])

parameter in the sample was $S=0.81$. The fit is shown in figure 23.

\section{Coherent phonons in graphene nanoribbons}

In this section, we extend the microscopic theory for generating and detecting coherent phonons in SWNTs to the case of coherent phonons in GNRs, although there are no measurements of coherent phonons to date in GNRs. Therefore, our discussion here gives some predictions of $\mathrm{CP}$ behavior in GNRs. We consider armchair and zigzag ribbons denoted $N_{a b}$ aGNR and $N_{a b}$ zGNR, respectively, where $N_{a b}$ is the number of $\mathrm{AB}$ carbon dimers in the unit cell.
The lattice structure for aGNRs and zGNRs is shown schematically in figure 24 . For both aGNRs and zGNRs there are $N_{a b} \mathrm{CP}$ active modes that vibrate in the plane of the nanoribbon. In all cases, the $\mathrm{CP}$ active mode with the lowest frequency is RBLM mode in which the nanoribbon width periodically expands and contracts. For coherent RBLM phonons the ribbon width $W(t)$ is directly proportional to the coherent phonon amplitude $Q(t)$ [47].

The RBLM phonon energies are sensitive to the ribbon width $W$ and scale roughly as the inverse of the ribbon width in accordance with a simple zone folding expression $E=0.4 / W \mathrm{eV}$ with $W$ in units of angstrom. The RBLM energies for aGNRs and zGNRs calculated using our valence force field model for GNR phonons are shown in figure 25 as functions of the ribbon width. We plot the RBLM phonon energies for $N_{a b}$ aGNRs for $4 \leq N_{a b} \leq 25$ as black dots and RBLM energies for $N_{a b}$ zGNRs for $3 \leq N_{a b} \leq 15$ as red triangles. To fit our calculated VFF energies as functions of the nanoribbon width, we fit our results to an empirical expression of the form $E=A W^{P}+B$ where $A, P$, and $B$ are fitting parameters the values of which are shown in figure 25 .

The coherent phonon driving function $S(t)$ immediately after photoexcitation is greatest for light polarized parallel to the nanoribbon axis. The sign of the driving function gives phase information that can be measured in $\mathrm{CP}$ spectroscopy.

\subsection{Armchair nanoribbons}

Armchair nanoribbons belong to one of three families depending on the mod number $\bmod \left(N_{a b}, 3\right)$. Based on a simple band structure calculation, we classify mod 0 and mod 1 aGNRs as semiconductors and mod 2 aGNRs as metals $[125,126]$.

Bandstructures for $\pi$ electrons in three representative armchair graphene nanoribbons (aGNRs) calculated in the ETB model are shown in figure 26. The $6 \mathrm{GNR}$ and 7 GNR ribbons are semiconducting with finite band gaps, while the mod $28 \mathrm{GNR}$ ribbon is metallic. Armchair semiconducting nanoribbons have direct gaps that arise from

\section{Armchair GNR (aGNR)}

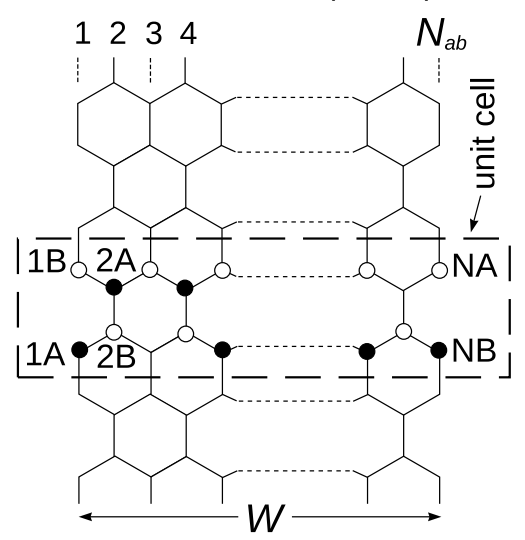

\section{Zigzag GNR (zGNR)}

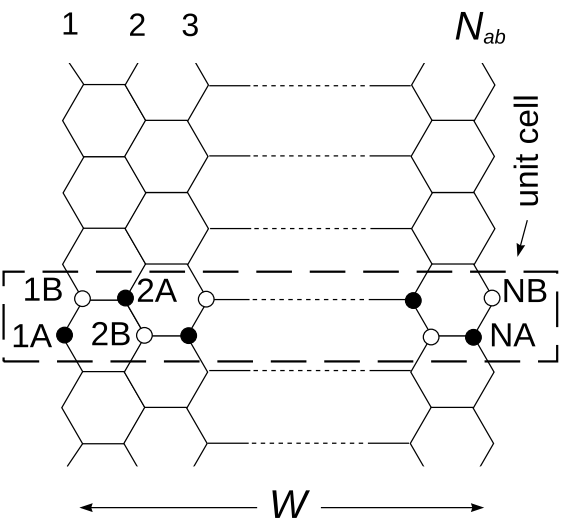

Figure 24. Lattice structures and translational unit cells for armchair (aGNR) and zigzag (zGNR) graphene nanoribbons. The width of the nanoribbons is $W$ (reproduced from [47]). 


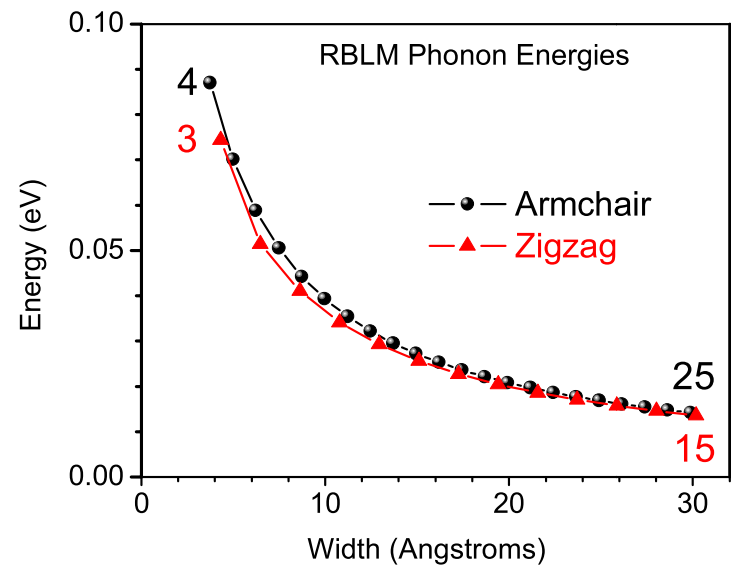

Fit to VFF results

$$
E=A W^{P}+B
$$

$$
\begin{array}{cc}
\text { Armchair } & \text { Zigzag } \\
A=.31376 & A=.25247 \\
P=-.95579 & P=-.82773 \\
B=.00328 & B=-.00136
\end{array}
$$

Zone Folding Approximation

$E_{R B L M}=0.4 \mathrm{eV} / \mathrm{W}\left(\mathrm{A}^{0}\right)$

Figure 25. RBLM phonon energies for armchair nanoribbons (black dots) and zigzag nanoribbons (red triangles) computed in the valence force field model as a function of ribbon width $W$. The RBLM phonon energies can be fit to $E=A W^{P}+B$ where $A, P$, and $B$ are fitting parameters. Each plot symbol corresponds to a different value of $N_{a b}$ which varies from 4 to 25 for armchair ribbons and from 3 to 15 for zigzag nanoribbons [47].
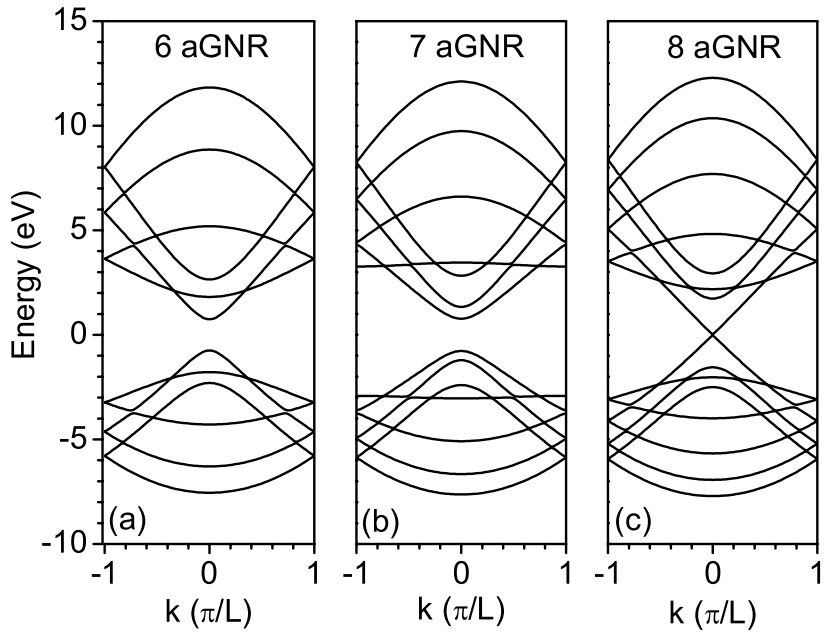

Figure 26. $\pi$ energy bands for 6,7 and 8 aGNR nanoribbons calculated in the ETB model (adapted from [47]).

quantum confinement and edge effects and all the electronic wavefunctions near the band edge are distributed throughout the width of the ribbon.

A typical mod 1 semiconducting nanoribbon is the 7 aGNR. In GNRs, the CP active mode with the lowest phonon energy is the RBLM mode at $q=0$. Figure 27 shows the 7 aGNR unit cell with 14 carbon atoms, and superimposed on these atoms are vectors proportional to the atomic displacements in the RBLM mode as determined in our valence force field model. As can be seen in the figure, the RBLM mode represents a periodic expansion and contraction of the ribbon width. The inset shows the phonon dispersion relations for out-of-plane modes (red curves) and in-plane phonon modes (black curves). The phonon branch containing the RBLM mode is shown as a thick black line and the RBLM mode at $q=0$ is indicated by a yellow dot. In the VFF model the RBLM phonon energy is found to be $51 \mathrm{meV}$.

Since coherent phonon spectroscopy gives direct phase information on the coherent phonon amplitude, it is thus instructive to examine $S_{\max }$ as a function of pump photon energy and nanotube species. This is done in figure 28 for $\bmod 0,1$ and 2 nanoribbons. Our results for the 6 aGNR mod 0 semiconducting nanoribbon are shown in figure 28(a) where $S_{\max }$ is shown as a function of pump photon energy. For comparison, the absorption coefficient is also plotted in the lower panel of figure 28(a). Near the band edge, we see from figure 28(a) that the pump light is strongly absorbed near the $E_{11}$ and $E_{22}$ peaks. The resulting increase in the photoexcited carrier density increases the coherent phonon driving function and enhances the coherent phonon oscillation amplitudes. Photoexcitation by the pump causes the nanoribbon to initially expand for pump photon energies near the $E_{11}$ transition and to initially contract for pump photon energies near the $E_{22}$ transition. We find this to be true for all mod 0 semiconducting aGNRs.

Qualitatively, different results are obtained for mod 1 aGNRs. In figure 28(b) we plot $S_{\max }$ as a function of pump photon energy for mod 17 aGNRs. Photoexcitation by the pump near the $E_{11}$ absorption peak causes the 7 aGNR to initially contract while pumping near the $E_{22}$ absorption peak causes the 7 aGNR to initially expand. This is true for all mod 1 semiconducting aGNRs. In figure 28(c) we show results for an 8 aGNR mod 2 metallic nanoribbon excited by a laser pulse polarized parallel to the ribbon length. From figure 28(c), we find that photoexcitation by the pump near the $E_{11}$ absorption peak causes the nanoribbon to initially expand. For photon energies near the $E_{22}$ transition, the situation is more ambiguous.

Again, we could explain the trend in figure 28 using effective mass theory to understand why some GNRs start their coherent RBLM oscillations by initially expanding while others start the oscillations by initially shrinking [47]. The $\operatorname{RBLM} \mathcal{H}^{(\mathrm{ep})}$ for an aGNR can be written as

$$
\mathcal{H}^{(\mathrm{ep})}=u_{\text {arm }}\left(\begin{array}{cc}
g_{\text {on }} & -\frac{g_{\text {off }}}{2} \\
-\frac{g_{\text {off }}}{2} & g_{\text {on }}
\end{array}\right),
$$



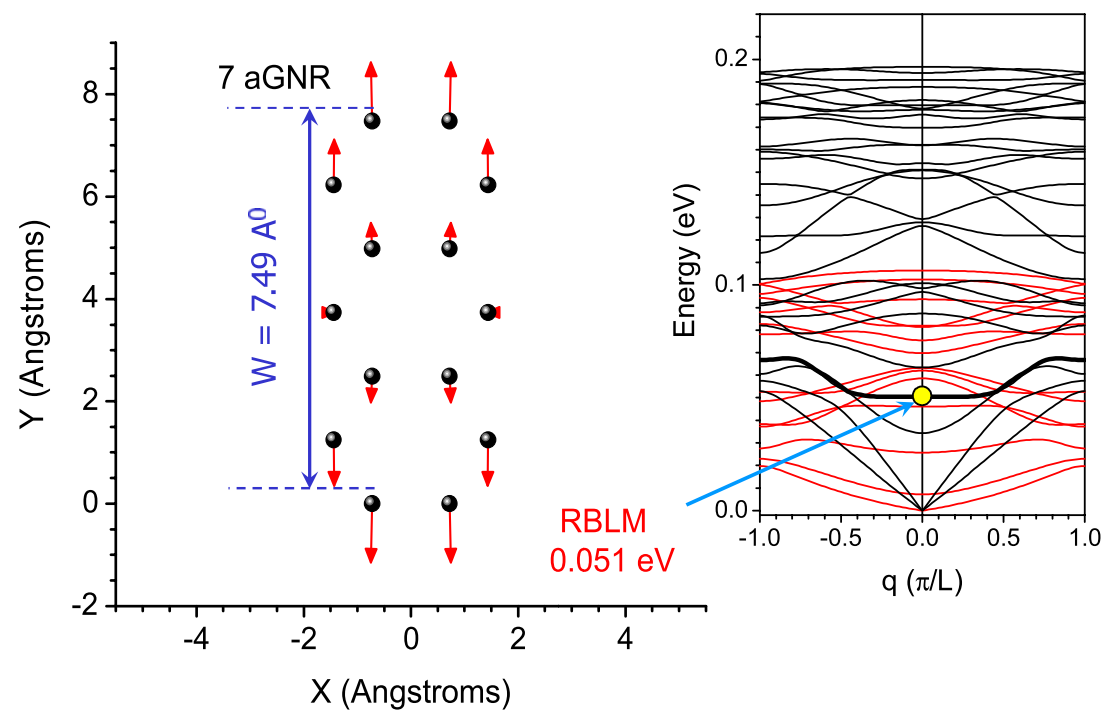

Figure 27. Phonon mode pattern for the RBLM mode (phonon energy $51 \mathrm{meV}$ ) in a 7 aGNR mod 1 semiconducting nanoribbon. The coherent phonon amplitude is proportional to the ribbon width and increasing amplitude corresponds to ribbon width expansion. The phonon dispersion relation is shown on the right with the phonon branch containing the RBLM mode at $q=0$ shown as a thick black line. The red lines correspond to out-of-plane modes and the black lines are in-plane modes.

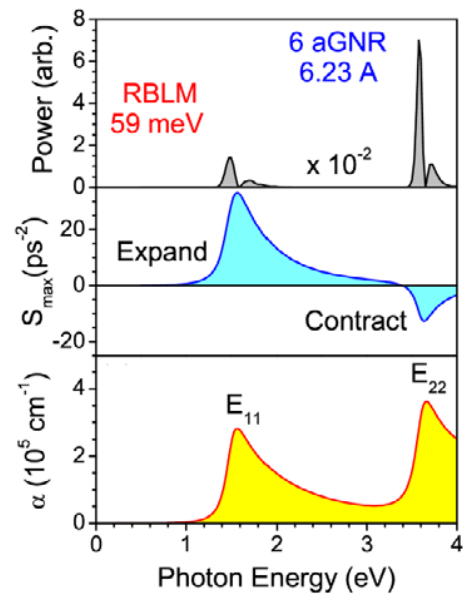

(a)

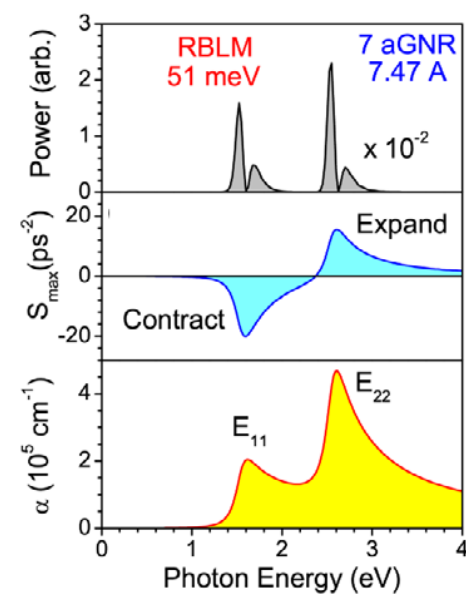

(b)

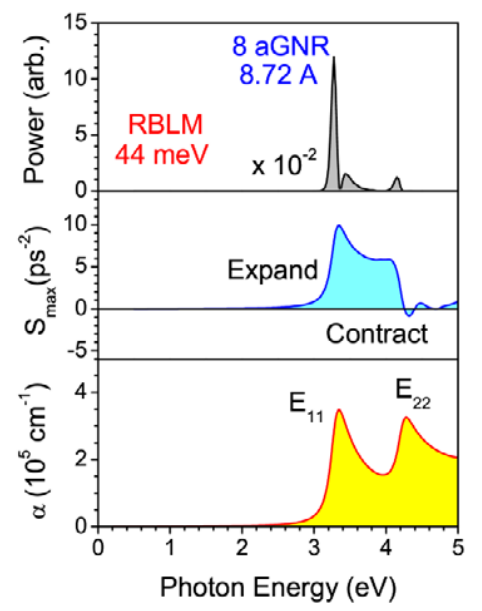

(c)

Figure 28. The coherent phonon power, the value of $S_{\max }$, and the initial absorption spectrum are plotted as a function of photon energy for (a) 6 aGNR mod 0 nanoribbon (RBLM frequency $=59 \mathrm{meV}$ ), (b) 7 aGNR $\bmod 1$ nanoribbon (RBLM frequency $=51 \mathrm{meV}$ ), and (c) 8 aGNR mod 2 nanoribbon (RBLM frequency $=44 \mathrm{meV}$ ). The excitation is due to a Gaussian laser pulse with pump and probe polarization vectors parallel to the ribbon length (reproduced from [47]).

where $g_{\text {on }}\left(g_{\text {off }}\right)$ is the on-site (off-site) coupling constant in electronvolt, while $u_{\mathrm{arm}}$ is a ribbon width- or $N_{a b}$-dependent phonon amplitude. To obtain the electron-phonon matrix element $M^{(\mathrm{ep})}$, we use the same wavefunctions as in (31). The wavefunctions are suitable near the graphene Dirac $\mathrm{K}$ point and thus they can explain well the aGNR lattice response especially at relatively low energy $E_{11}$ and $E_{22}$ optical transitions. We then obtain a formula for aGNR $M^{(\mathrm{ep})}$ similar to that for the nanotube case,

$$
M^{(\mathrm{ep})}=-u_{\text {arm }}\left(2 g_{\text {off }} \cos \Theta(\mathbf{k})\right) .
$$

Here $\Theta(\mathbf{k})$ is defined by the angle that $\mathbf{k}$ points in the two-dimensional Brillouin zone measured from the line in $\mathbf{k}$ space perpendicular to the discrete one-dimensional Brillouin zone (cutting lines) which goes over the $\mathrm{K}$ point (hexagonal corner, see figure 29). Therefore, the driving function kernel can be written as [47]

$$
S(\mathbf{k})=\frac{2 \omega_{\text {RBLM }}}{\hbar} u_{\text {arm }}\left[2 g_{\text {off }} \cos (\Theta(\mathbf{k}))\right],
$$

where $\omega_{\mathrm{RBLM}}$ is the RBLM frequency at $q=0$. From this equation, we can analyze the $N_{a b}$ and $E_{i i}$ dependence of the aGNR initial lattice response. First of all, we should note that $g_{\text {off }}$ and $u_{\text {arm }}$ are always positive, while $\cos \Theta(\mathbf{k})$ can either be positive or negative depending on the value of $\mathbf{k}$ at which the $E_{i i}$ transition occurs.

Using this argument, we can classify the aGNR lattice response based on the aGNR types. For example, let us 


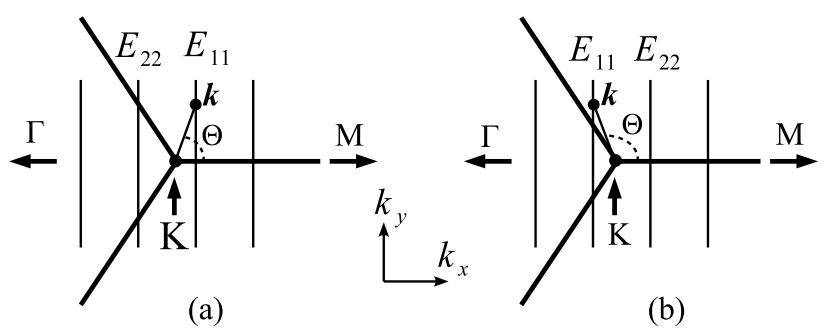

(a)

(b)

Figure 29. Cutting lines for (a) $\bmod 0$ aGNR and (b) $\bmod 1$ aGNRs near the Dirac K point. To make clear the definition of $\Theta(\mathbf{k})$, in this figure $\Theta(\mathbf{k})$ is shown for an arbitrary $\mathbf{k}$ at $E_{11}$. In fact, in the case of $\bmod 0$ and $\bmod 1$ aGNRs the $E_{11}$ transitions occur at $\Theta(\mathbf{k})=0$ and $\pi$, respectively. The difference between the mod 0 and $\bmod 1$ aGNRs can be understood from the position of the $E_{11}$ or $E_{22}$ cutting lines relative to the K point (reproduced from [47]).

consider semiconducting mod 0 aGNR and mod 1 aGNRs. The cutting line position for their $E_{11}$ and $E_{22}$ optical transitions are just opposite to each other. For a mod 0 aGNR, we see that $\cos \Theta(\mathbf{k})$ becomes positive (negative) at $E_{11}\left(E_{22}\right)$, and thus the aGNR starts the coherent phonon oscillations by expanding (shrinking) its width. This can be seen in the illustration of $\Theta(\mathbf{k})$ in figure 29. The opposite behavior is true for mod 1 aGNRs.

The effective mass predictions for semiconducting mod 0 and mod 1 aGNRs are borne out by the ETB coherent phonon spectroscopy simulations summarized in figures 30 and 31 . In the case of mod 0 aGNRs, pumping at the $E_{11}$ transition causes the nanoribbon to initially expand while pumping at the $E_{22}$ transition causes the nanoribbon to initially contract. The exact opposite is true in the case of mod 1 aGNRs. For higher lying transitions, the effective mass theory breaks down as these transitions are too far from the Dirac points.

The driving force trends for mod 2 metallic aGNRs (see figure 32) cannot be explained by the effective mass theory. In metallic aGNRs, two cutting lines are equidistant from the $\mathrm{K}$ point and are the lower and higher branches of an $E_{i i}$ transition. Both branches contribute to $E_{i i}$ and we sum up the matrix elements from each contribution to obtain $M^{(\mathrm{ep})}$. For example, if the 1D $k$-points for the lower and higher branches of $E_{i i}$ are the same, the matrix elements cancel because $\cos \Theta(\mathbf{k})+\cos (\pi-\Theta(\mathbf{k}))=0$. In this case, the $\mathrm{CP}$ amplitude will be small for the mod 2 metallic aGNRs as compared to the mod 0 or mod 1 semiconducting aGNRs. In reality, we have slightly different $k$-points for the two $E_{i i}$ branches due to trigonal warping effects [36]. When trigonal warping effects are included, the resulting nonzero value of $M^{(\mathrm{ep})}$ allows us to determine if the ribbon width initially expands or contracts.

Near the $E_{11}$ transition in metallic aGNRs the lattice initially expands for all $N_{a b}$. Near the $E_{22}$ transition, the lattice always initially contracts, though we see in figures 32(b)-(d) the trends do not hold for larger $N_{a b}$. We summarize the lattice behavior at $E_{11}$ and $E_{22}$ transitions for all families of aGNRs in table 2 .

\subsection{Zigzag nanoribbons}

The molecular structure of a zigzag graphene nanoribbon (zGNR) is shown in figure 24. All zGNRs are metallic and there is no classification into qualitatively distinct types like there is in armchair nanoribbons. Furthermore, the bands for

\section{Mod 0 ETB Results}
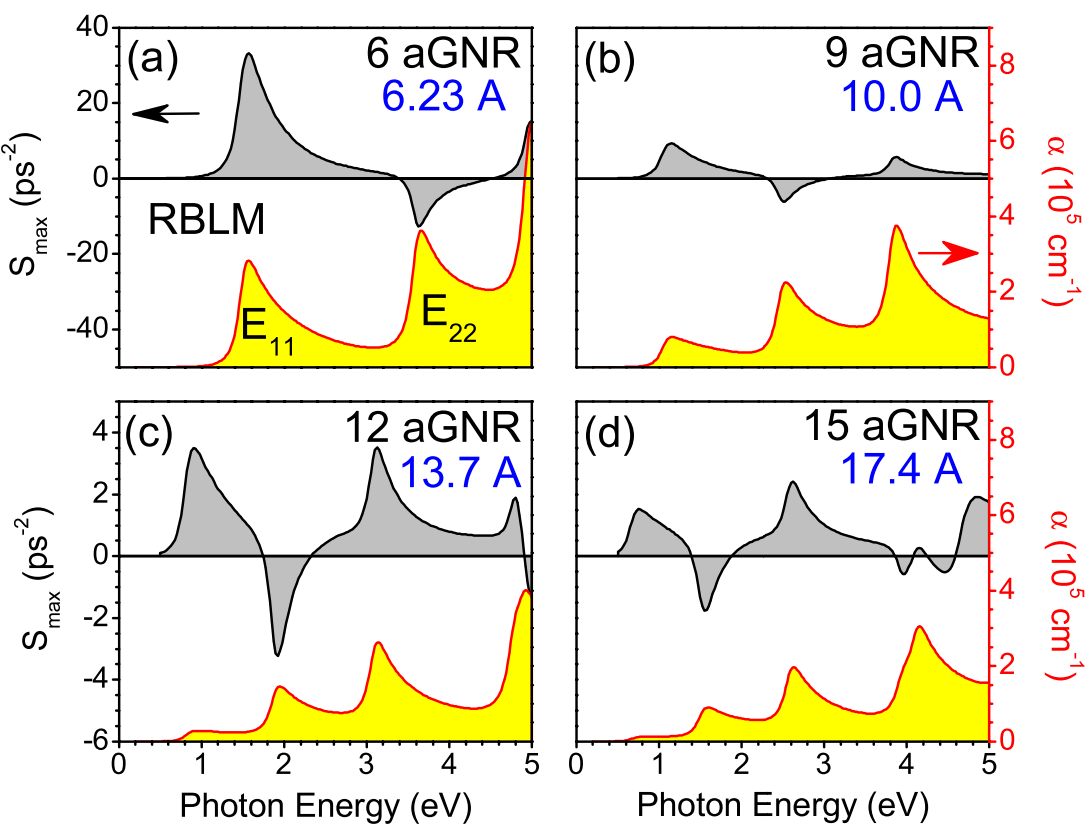

Figure 30. RBLM coherent phonon driving function and initial absorption spectrum as a function of photon energy for several mod 0 semiconducting aGNRs: (a) $N_{a b}=6$, (b) $N_{a b}=6$, (c) $N_{a b}=12$, and (d) $N_{a b}=15$. Positive (negative) $S_{\max }$ at $E_{11}\left(E_{22}\right)$ corresponds to an expansion (contraction) of the ribbon width (adapted from [47]). 

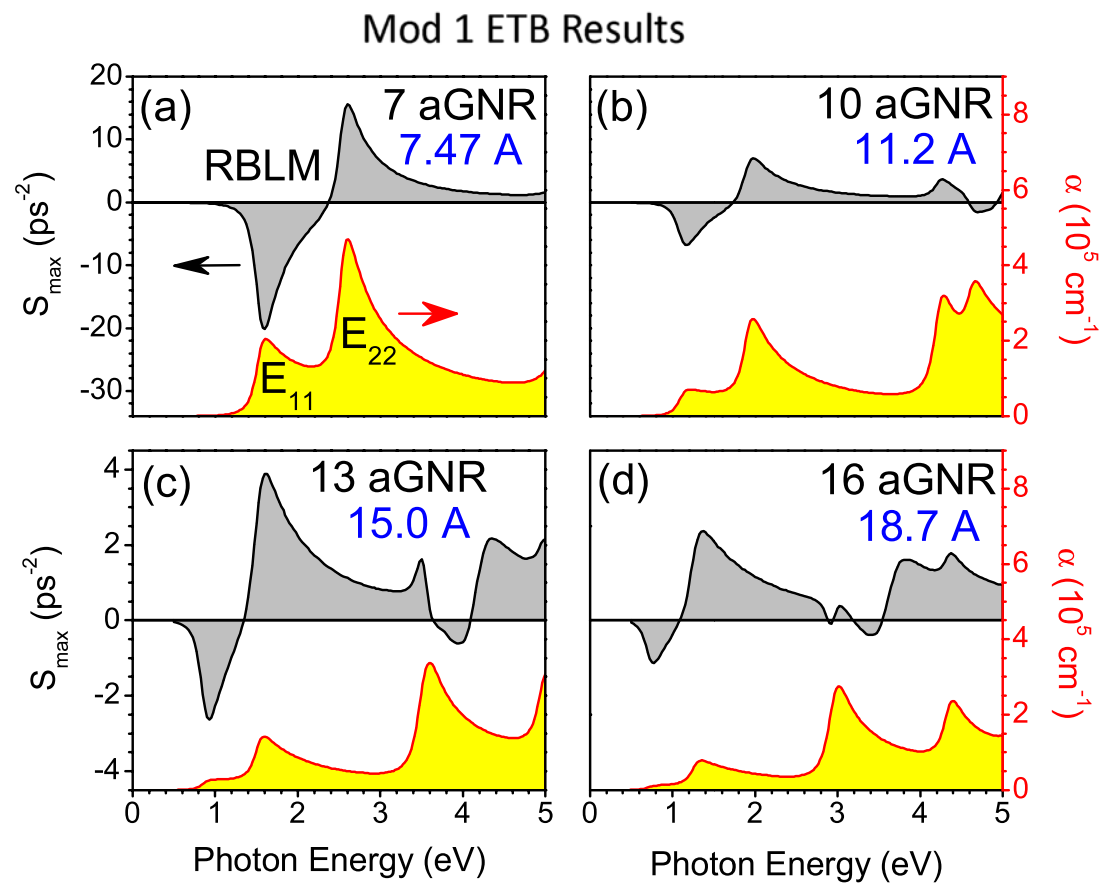

Figure 31. RBLM coherent phonon driving function and initial absorption spectrum as a function of photon energy for several mod 1 semiconducting aGNRs: (a) $N_{a b}=7$, (b) $N_{a b}=10$, (c) $N_{a b}=13$, and (d) $N_{a b}=16$. Negative (positive) $S_{\max }$ at $E_{11}\left(E_{22}\right)$ corresponds to a contraction (expansion) of the ribbon width (adapted from [47]).

Mod 2 ETB Results
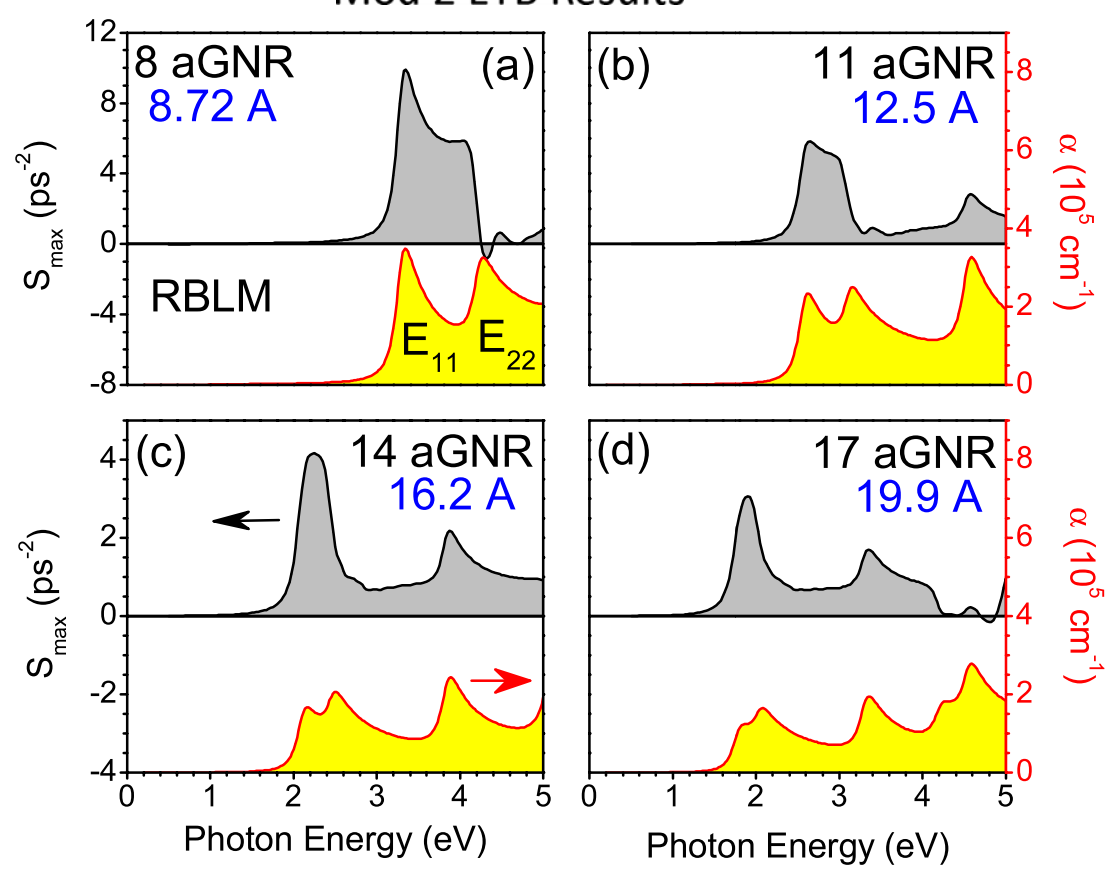

Figure 32. RBLM coherent phonon driving function and initial absorption spectrum as a function of photon energy for several mod 2 metallic aGNRs: (a) $N_{a b}=8$, (b) $N_{a b}=11$, (c) $N_{a b}=14$, and (d) $N_{a b}=17$. Positive (negative) $S_{\max }$ at $E_{11}$ ( $E_{22}$ ) corresponds to an expansion (contraction) of the ribbon width (adapted from [47]).

Table 2. Initial lattice behavior due to coherent phonon oscillations at $E_{11}$ and $E_{22}$ in aGNRs.

\begin{tabular}{lll}
\hline Family & $E_{11}$ & $E_{22}$ \\
\hline mod 0 & Expand & Contract \\
mod 1 & Contract & Expand \\
mod 2 & Expand & Expand or contract \\
\hline
\end{tabular}

all zGNR nanoribbons are qualitatively similar. Thus when we discuss zGNR electronic states it is sufficient to consider just one example.

It is mentioned, however, that the localized electronic energy band, which are the so called edge states as we will discuss below, exists for zGNR in which the $\mathrm{C} 1$ and V1 energy bands as shown in figure 33 are merged into degenerate energy 


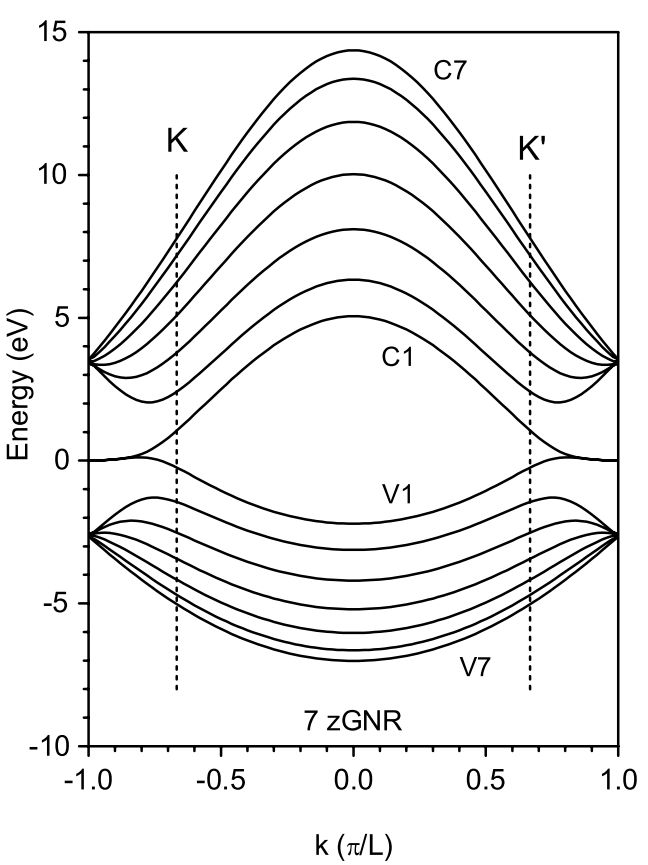

Figure 33. $\pi$ energy bands for 7 zGNR nanoribbons calculated in the ETB model. The lowest seven bands labeled $V 1 \cdots V 7$ are the valence bands and the highest seven bands labeled $C 1 \cdots C 7$ are the conduction bands.

bands at the zone boundary region. Since the contribution of the edge states to CP amplitude is not clear, we did not discuss the calculation in [47]. The discussion here is thus an original results in this review article.

The computed bandstructure for $\pi$ bands in 7 zGNR nanoribbons is shown in figure 33. The Brillouin zone is one dimensional with $|k| \leq \pi / L$ and there are fourteen bands. The lowest seven bands labeled V1, ., V7 are the valence bands and the remaining bands labeled $\mathrm{C} 1, \ldots, \mathrm{C} 7$ are the conduction bands. We note that the conduction and valence bands are asymmetric about $E=0$ because of atomic overlap matrix elements in the ETB formalism which are not present in simple tight-binding (STB) and effective mass models. There is a strong degeneracy in the bands at $k= \pm \pi / L$ and there are two partially degenerate bands V1 and $\mathrm{C} 1$ near $E=0$. These ground state bands correspond to localized edge states for $|k| \gtrsim 2 \pi / 3 L$ where $k= \pm 2 \pi / 3 L$ are the Dirac points $\mathrm{K}$ and $\mathrm{K}^{\prime}$. For $|k| \lesssim 2 \pi / 3 L$ the states penetrate into the interior of the ribbon as $|k| \rightarrow 0$. These edge states are peculiar to zigzag nanoribbons and have been studied using effective mass, tight-binding and $a b$ initio theories [127-129]. The remaining bands are delocalized zone folded quantum confined bands the lowest of which have parabolic minima near the Dirac points $K$ and $K^{\prime}$ located at $k= \pm 2 \pi / 3 L$.

Figure 34(a) shows a closeup of the ETB $\pi$ band states near the band edge for 7 zGNR nanoribbons. The Fermi level at a temperature of $300 \mathrm{~K}$ is $E_{\mathrm{f}}=0.07 \mathrm{eV}$ and is shown as a dotted line. Figures 34(b) and (c) respectively show differences in the STB and ETB energy bands in the vicinity of the $\mathrm{K}^{\prime}$ point. In the STB model, the bands are symmetric about $E=0$ and in the ETB model, the bands are asymmetric. Thus, in the STB model, the Fermi energy for undoped GNRs is pinned at $E_{\mathrm{f}}=0 \mathrm{eV}$ independent of the temperature while the Fermi energy in the ETB model is temperature dependent.

This difference in band curvature near $\mathrm{K}$ and $\mathrm{K}^{\prime}$ has pronounced effects on band edge absorption. In zGNRs, we designate optical transitions between bands $C_{i}$ and $V_{j}$ with the obvious notation $V_{i} C_{j}$. Optical absorption in GNRs is qualitatively different from absorption in SWNTs. GNRs have a vertical mirror plane running down the center so the electronic states are either symmetric or antisymmetric about this mirror plane. Optically dipole allowed transitions for polarization parallel to the nanoribbon axis are between states in bands with the same parity and discrete $k$ values (or the same cutting line). The edge states $\mathrm{V} 1$ and $\mathrm{C} 1$ near the Brillouin zone boundary play an important role in the band edge transitions and the absorption spectrum is sensitive to the Fermi energy.
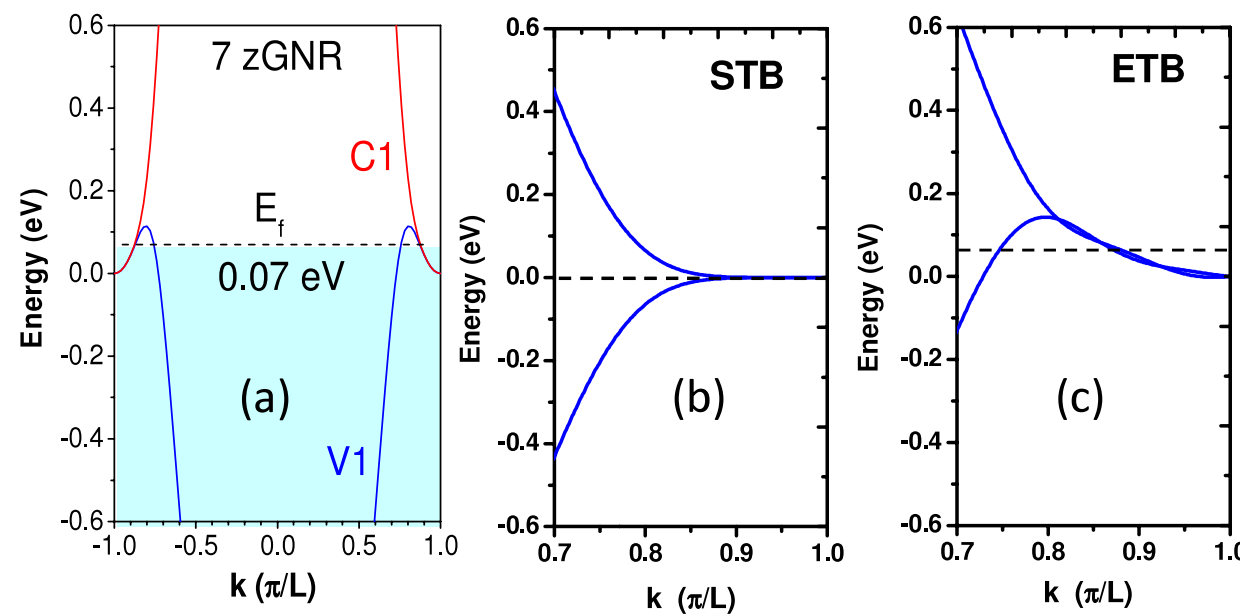

Figure 34. A closeup of $\mathrm{C} 1$ and $\mathrm{V} 1$ band edge states in undoped 7 zGNR nanoribbons computed using the ETB model is shown in (a). The Fermi level at $300 \mathrm{~K}$ is $0.07 \mathrm{eV}$ and is indicated by the dotted line. The STB and the ETB bands in the vicinity of the $\mathrm{K}^{\prime}$ point are shown in (b) and (c), respectively with dotted lines indicating the Fermi level in the two models. 

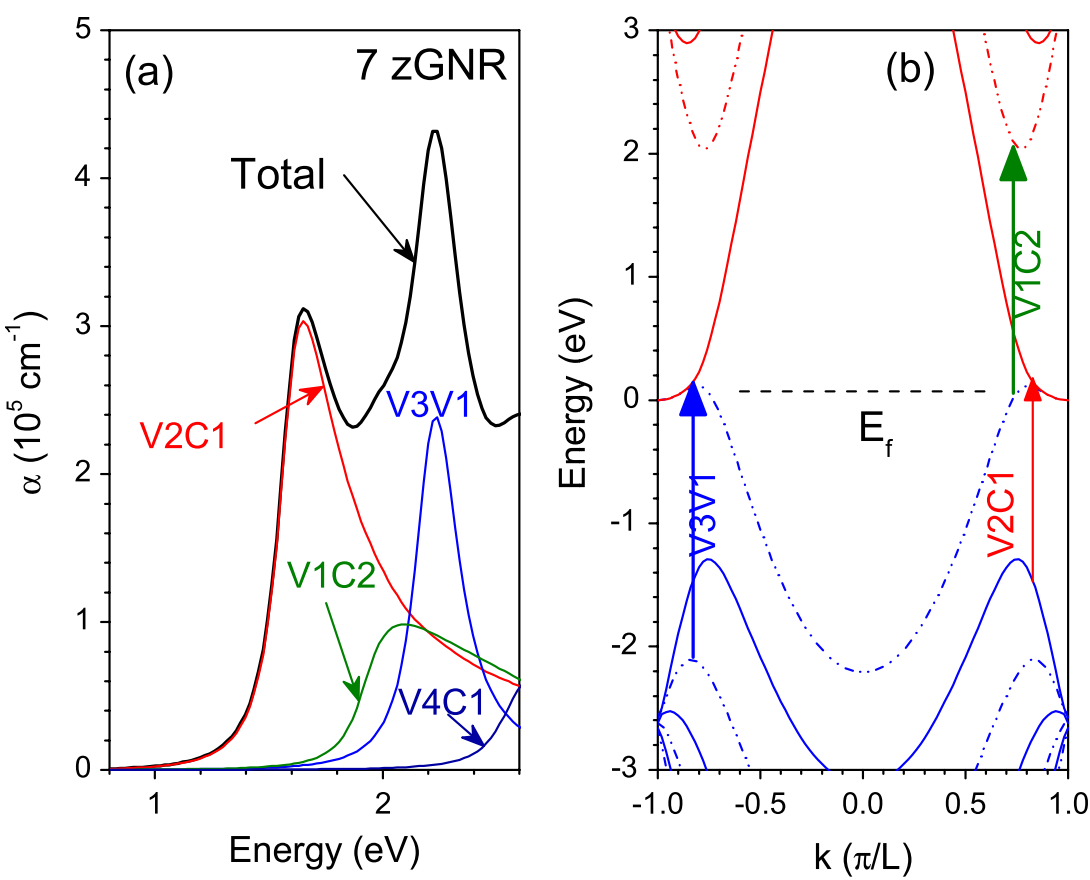

Figure 35. (a) Band edge absorption for 7 zGNR nanoribbons with electric polarization vector parallel to the ribbon. Total absorption is the sum of absorption due to several transitions. (b) Band diagram showing the Fermi level $E_{\mathrm{f}}$ and transitions involved in the three lowest absorption peaks. Blue lines are valence bands and red lines are conduction bands. Solid lines $(n$ even) are even parity states and the dash-dotted lines ( $n$ odd) are odd parity states. (reproduced from [47]).

The absorption spectrum for the 7 zGNR nanoribbon is shown in figure 35(a) for linearly polarized light with the electric polarization vector parallel to the nanoribbon axis. The nanoribbon is assumed to be undoped at a temperature of $300 \mathrm{~K}$. The Fermi level $E_{\mathrm{f}}$ at $70.16 \mathrm{meV}$ is indicated by the black dashed line in figure 35(b). The V1 edge states in the vicinity of the Dirac points lie above the Fermi energy and since these states are empty, electrons in the lower valence bands can be photoexcited into these V1 states thus accounting for the strong intraband V3V1 absorption peak near $2.23 \mathrm{eV}$. The Fermi golden rule absorption coefficient is computed with a FWHM linewidth of $0.2 \mathrm{eV}$. The total absorption spectrum shown as a thick black line is a sum of contributions from several transitions. The lowest lying absorption peak at $1.65 \mathrm{eV}$ is $\mathrm{V} 2 \mathrm{C} 1$ and is indicated by a vertical arrow in figure $35(\mathrm{~b})$. The $\mathrm{V} 2 \mathrm{C} 1$ peak comes from transitions near $\mathrm{K}$ and $\mathrm{K}^{\prime}$. The initial states are quantum confined hole states V2 and the final states are the localized electron edge states $\mathrm{C} 1$. A second broad absorption transition $\mathrm{V} 1 \mathrm{C} 2$ peaking at $2 \mathrm{eV}$ comes from transitions between the localized hole edge states V1 and the second quantum confined electron states $\mathrm{C} 2$. A strong peak in the absorption spectrum at $2.23 \mathrm{eV}$ is due to the intraband transition V3V1 between $\mathrm{V} 3$ quantum confined states near the $\mathrm{K}$ and $\mathrm{K}^{\prime}$ points and the localized V1 hole edge states.

Figure 36 shows the 7 zGNR unit cell with 14 carbon atoms, and superimposed on these are vectors proportional to the atomic displacements in the RBLM mode as determined in our valence force field model. Again, the RBLM mode represents a periodic expansion and contraction of the ribbon width. The inset shows the phonon dispersion relations for out-of-plane modes (red curves) and in-plane phonon modes (black curves). The phonon branch containing the RBLM mode is shown as a thick black line and the RBLM mode at $q=0$ is indicated by a yellow dot. In the VFF model the RBLM phonon energy is found to be $29 \mathrm{meV}$.

For photoexcitation near the optical absorption edge, the RBLM driving function is much larger for zGNRs in which transitions involving localized edge states provide the dominant contribution. Figure 37(a) is the power spectrum of $\mathrm{CP}$ amplitude $Q(t)$ at the RBLM frequency. In figure 37(b) we plot $S_{\max }$ as a function of pump photon energy. The absorption coefficient is plotted in figure 37(c) for comparison. Near the band edge, we see from figure 37(b) that the pump light is strongly absorbed at the $\mathrm{V} 2 \mathrm{C} 1$ and $\mathrm{V} 3 \mathrm{~V} 1$ peaks. The resulting increase in the photoexcited carrier density increases the coherent phonon driving function and enhances the coherent phonon oscillation amplitudes. In other words the coherent phonon driving function near the band edge is determined by the strength of optical absorption between the lowest few hole bands and the localized edge states V1 and C1. At energies above $3 \mathrm{eV}, S_{\max }$ changes sign and the nanoribbon initially contracts.

We performed simulations of the generation and detection of RBLM coherent phonons in zGNRs with $N_{a b}$ ranging from 6 to 14. In figure 38 we plot the maximum driving function $S_{\max }$ as black curves against the left axis and the absorption coefficient (red curves) against the right axis. We assume the pump and probe are polarized parallel to the ribbon axis. In all cases, the maximum driving function $S_{\max }$ is positive (and the ribbon width initially expands) for energies below approximately $3 \mathrm{eV}$ and is negative for higher energies. 


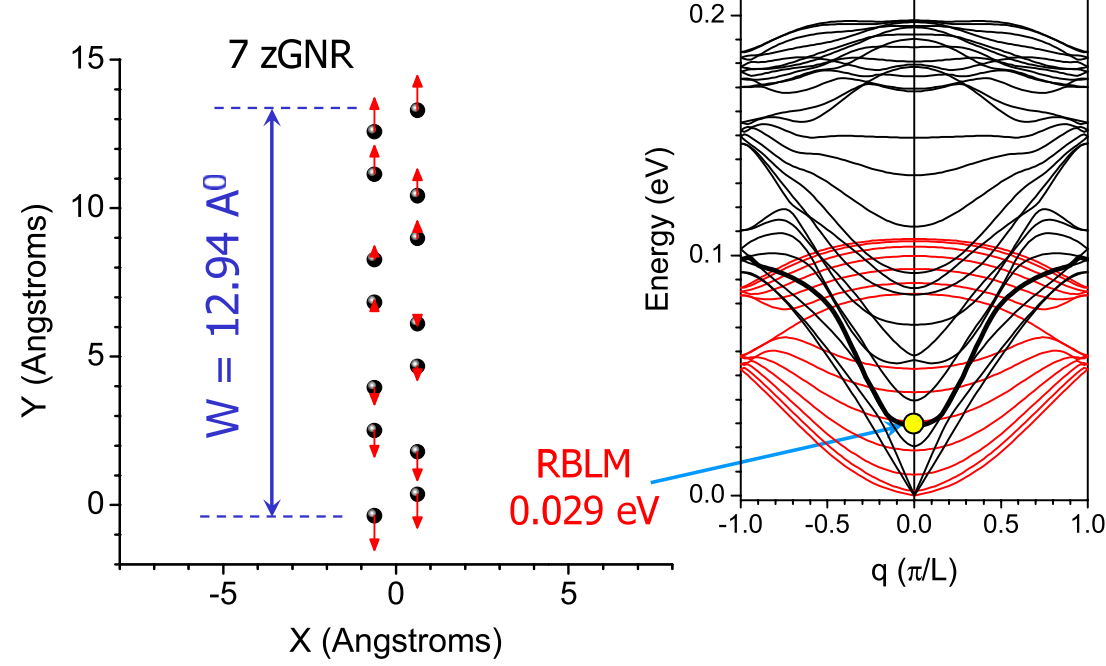

Figure 36. Phonon mode pattern for the RBLM mode (phonon energy $29 \mathrm{meV}$ ) in a $7 \mathrm{zGNR}$ nanoribbon. The coherent phonon amplitude is proportional to the ribbon width with increasing amplitude corresponding to ribbon width expansion. The phonon dispersion relation is shown on the right with the phonon branch containing the RBLM mode at $q=0$ shown as a thick black line. The red lines correspond to out-of-plane modes and the black lines are in-plane modes.

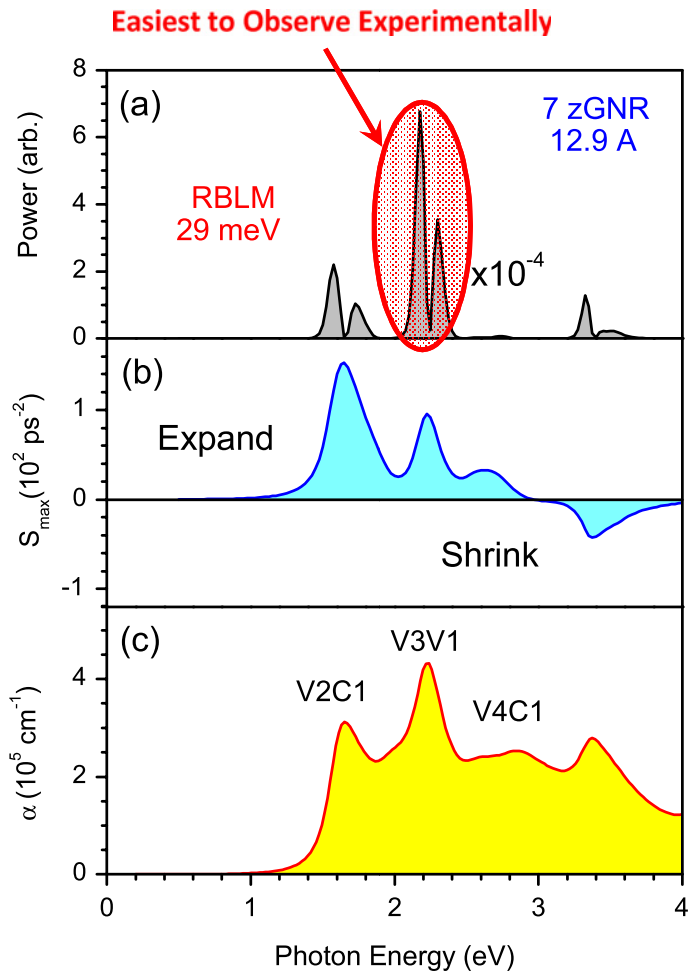

Figure 37. For 7 zGNR nanoribbon excited by Gaussian a laser pulse with polarization vector parallel to ribbon length, we plot (a) the coherent phonon power at the RBLM frequency $(29 \mathrm{meV})$, (b) the value of $S_{\max }$, and (c) the initial absorption spectrum as a function of photon energy (adapted from [47]).

The V3V1 feature near $2 \mathrm{eV}$ has the strongest $\mathrm{CP}$ intensity and is judged easiest to observe.

The CP intensity as a function of nanoribbon width is shown in figure 39 where the pump and probe polarization are parallel to the nanoribbon width. In figure 39 the coherent
RBLM phonon intensity for the V2C1 and V3V1 transitions as a function of $N_{a b}$ is plotted against the left axis and the RBLM frequency is plotted against the right axis. We have studied all zGNR nanoribbons for $N_{a b}$ ranging from 6 to 17 and find similar results. We see that the coherent RBLM amplitudes of zGNRs quickly decrease with increasing ribbon width.

We also investigated the in-plane polarization dependence of the coherent phonon spectra for 7 GNRs [47]. The polarization of the electric field of both pump and probe are rotated by an angle $\theta$ with respect to the nanoribbon length as indicated schematically in figure 40(a). The dependence of the RBLM CP power spectrum on $\theta$ is shown in figure 40(b) where $\mathrm{CP}$ power spectra are shown for $\theta$ ranging from $0^{\circ}$ and $50^{\circ}$. For $\theta=0^{\circ}$, there is a strong V $3 \mathrm{~V} 1$ signal at $2.25 \mathrm{eV}$ and a weaker $\mathrm{V} 2 \mathrm{C} 1$ signal at $1.65 \mathrm{eV}$. As $\theta$ increases from $0^{\circ}$ to $50^{\circ}$, the $\mathrm{CP}$ signal becomes weaker. The $\mathrm{CP}$ intensity is plotted on a log scale in figure 40 (c) for $\mathrm{V} 2 \mathrm{C} 1$ and $\mathrm{V} 3 \mathrm{~V} 1$ transitions. As $\theta$ varies from $0^{\circ}$ to $90^{\circ}$, the $\mathrm{CP}$ intensity is strongly quenched.

To apply the effective mass theory in zGNRs, however, we have to consider a special localized edge state, which is different with the wavefunctions defined in equation (31). Either conduction or valence band can be the initial or final localized edge state labeled as the first subband index. For examples, in 7zGNR, V3V1 transition means that the edge state is $\mathrm{V} 1$ as the final state, $\mathrm{V} 2 \mathrm{C} 1$ means that the edge state is $\mathrm{C} 1$ as the final state, and $\mathrm{V} 1 \mathrm{C} 2$ means that the edge state is $\mathrm{V} 1$ as the initial state. An appropriate definition of the edge state wavefunction should take into account the localization along the zigzag edge. Assuming that the zGNR width is along the $x$-axis, Sasaki et al proposed such a wavefunction defined as follows [130],

$$
\Psi_{\mathrm{e}}=N^{\prime} \mathrm{e}^{\mathrm{i} k_{y} y} \mathrm{e}^{-G(x)}\left(\begin{array}{l}
\mathrm{e}^{+g(x)} \\
\mathrm{e}^{-g(x)}
\end{array}\right),
$$




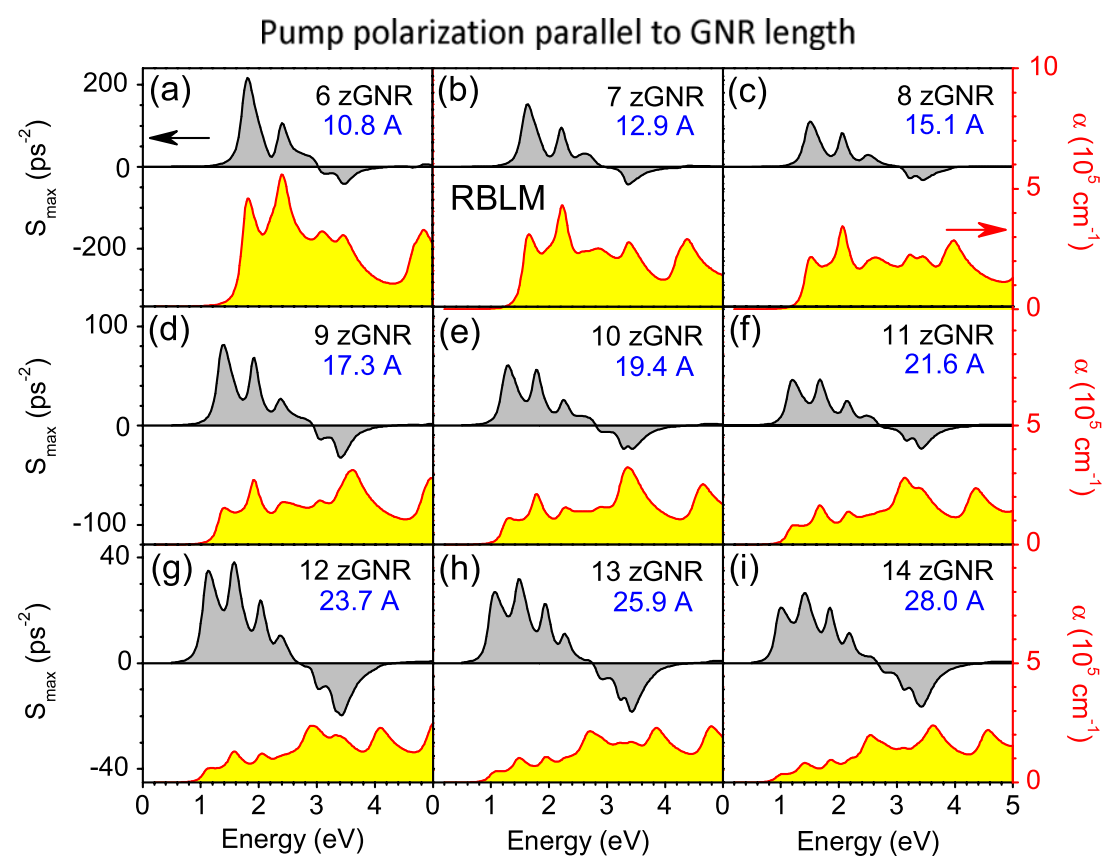

Figure 38. For zigzag nanoribbons excited by Gaussian laser pulse with pump and probe polarization parallel to ribbon lengths, we plot the maximum driving function $S_{\max }$ (black curves) against the left axis and the initial absorption coefficient (red curves) against the right axis. We consider all zGNRs with $N_{a b}$ ranging from 6 to 14. The nanoribbon widths in angstroms are shown in blue for each zGNR.

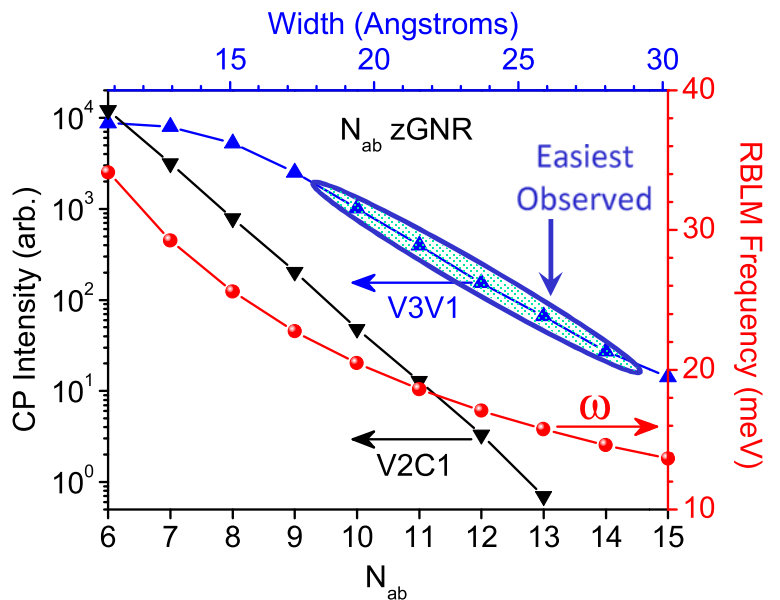

Figure 39. For zigzag nanoribbons excited by Gaussian laser pulse with pump and probe polarization parallel to ribbon length, we plot the coherent RBLM phonon intensity for V2C1 and V3V1

transitions as a function of $N_{a b}$, the number of carbon dimers in the zigzag unit cell, on the left axis. On the right axis we plot RBLM frequency $\omega$ in electronvolt. The ribbon width can be read from the upper axis (adapted from [47]).

where $N^{\prime}$ is a normalization constant, $\mathrm{e}^{-G(x)}$ is the amplitude of the wavefunction with some localization length, and $g(x)$ is the pseudospin modulation part. Detailed discussion about the properties of this wavefunction is given in [130].

Now, the two contributions in the electron-phonon matrix element in (30) in the case of zGNRs should be separated between the valence and conduction states. One of the two states should be the edge state (37), while another one should be the normal state (31). Denoting the matrix element for the (a)
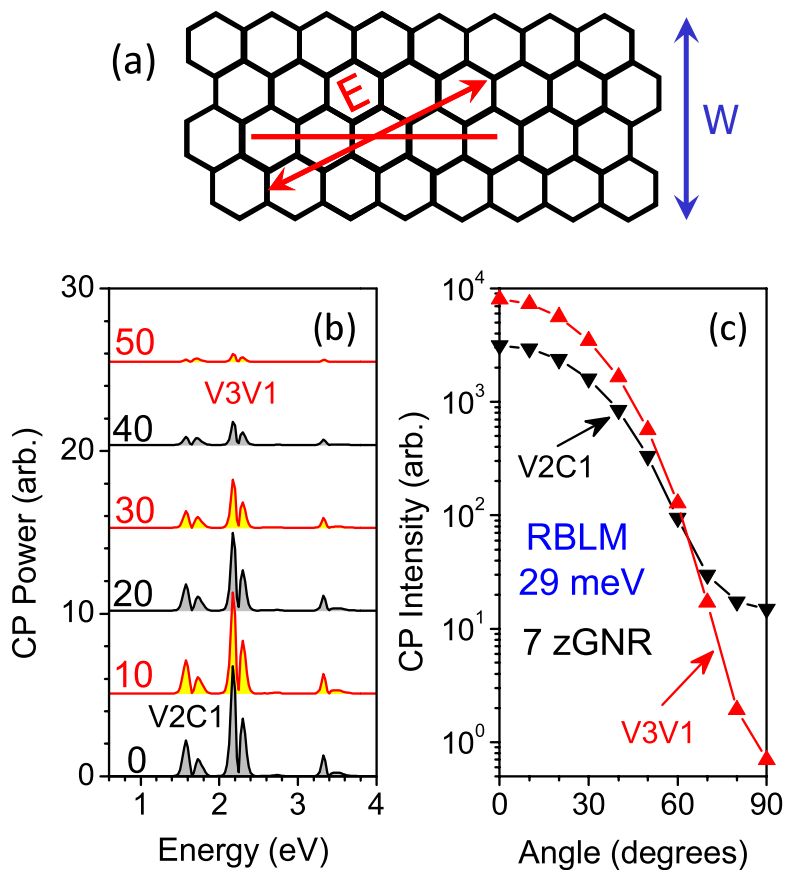

Figure 40. The pump-probe polarization angle $\theta$ relative to the zGNR nanoribbon axis is shown schematically in (a). For 7 zGNRs excited by Gaussian laser pulse we plot (b) the RBLM CP spectrum as a function of pump/probe energy for $\theta$ ranging from $0^{\circ}$ to $50^{\circ}$ and (c) the integrated RBLM CP intensity for V2C1 and V3V1 transitions as functions of $\theta$ (adapted from [47]).

normal state as $M_{b}^{(\mathrm{ep})}$ ( $b$ could be conduction state c or valence state $v)$, we find that

$$
M_{b}^{(\mathrm{ep})}=-u_{\mathrm{zig}}\left(g_{\text {off }} \cos \Theta(\mathbf{k})\right),
$$


where $u_{\text {zig }}$ is the zGNR RBLM zero-point phonon amplitude. On the other hand, after some algebra for the edge state, we find that $M_{e}^{(\mathrm{ep})}$, where $e$ can also be either $\mathrm{c}$ or $\mathrm{v}$, is just proportional to $u_{\mathrm{zig}}$.

$$
M_{e}^{(\mathrm{ep})}=C_{e} u_{\mathrm{zig}},
$$

where $C_{e}$ is a large constant. This means that the expansion or contraction of zGNR width is determined by the normal state (38), while the amplitude might be strongly affected by the edge state since $u_{\mathrm{zig}}$ is decreasing by increasing $N_{a b}$ of zGNR. This result is thus consistent with the results in figures 37 and 39 .

\section{Summary and conclusions}

In this paper we have reviewed our theoretical method for calculating the coherent phonon spectrum in carbon nanotubes and graphene nanoribbons. Calculation of the coherent phonon spectrum requires a detailed knowledge of (i) the electronic structure, (ii) optical matrix elements, (iii) phonon modes and (iv) electron-phonon matrix elements. In our method, we have developed a microscopic theory which uses a tight-binding model for the electronic states and optical matrix elements and a valence force field model for the phonons. Combining the phonon valence force field model with the extended tight-binding model for electronic states allows us to determine the electron-phonon matrix elements.

Our results show that the coherent RBM (of SWNTs) and RBLM (of GNRs) phonon spectrum strongly depend on tube chirality and ribbon type. In addition, we find the phase of the amplitude (i.e. whether the tube diameter or ribbon width initially expand or contract) can vary depending on the tube chirality or ribbon type. Comparison of our microscopic theory with a simplified effective mass theory provides an explanation of the initial contraction or expansion. Using effective mass theory for the electron-phonon interactions, we can analytically analyze how the tube diameter changes in response to femtosecond laser excitation and under what conditions the tube diameter or the ribbon width will initially increase or decrease. Results show that the initial phase of the coherent phonon oscillation depends on the relative position of the $E_{11}$ and $E_{22}$ cutting lines with respect to the K point.

We have compared our theoretical calculations for the SWNT to two different types of experiment: (i) micelle suspended nanotubes and (ii) films of horizontally aligned tubes. In micelle suspended samples, comparison to experiment is difficult because the experimental samples consist of a distribution of randomly oriented nanotubes with distribution of different types and chiralities. For micelle suspended tubes, one can use femtosecond pulse shaping to selectively isolate a specific SWNT chirality. In this case, we can analyze a specific chirality and our model predicts the overall trends in the relative strengths of the $\mathrm{CP}$ signal both within and between different $(2 n+m)$ families. For horizontally aligned nanotube films, we studied the polarization dependence of the pump and probe pulse with respect to the nanotube film axis. For the aligned films, two types of experiments were modeled. In Geometry 1 experiments, both the pump and probe polarizations were rotated with respect to the tube axis. In Geometry 2 experiments, only the pump polarization was rotated and the probe polarization was fixed along the tube axis. We found reasonable agreement between our calculations and the experiment predicting the overall dependence on the $\mathrm{CP}$ spectrum with the angle of the polarization. However, our results suggested that the nanotube films were not perfectly aligned, and allowing for a distribution of tube alignments produced better agreement between theory and experiment.

In the case of GNRs, experiments have not been performed to date. We theoretically find however, that for photoexcitation near the optical absorption edge, the CP driving term for the RBLM is much larger for zigzag nanoribbons where the strong transitions between localized edge states provide the dominant contribution to the $\mathrm{CP}$ driving term. We eagerly await future experiments to be performed in this area.

Perhaps the most important correction that can be made to our model in the future is to include the Coulomb interactions between photoexcited electron and hole pairs (exciton effects). Exciton effects are quite strong in carbon nanotubes with binding energies as large as $0.5 \mathrm{eV}$ or even larger for nanotubes with diameters less than $0.7 \mathrm{~nm}$. We would expect that the dominant effect of excitons would be to change the electronic states and optical matrix elements. This would affect where the initial photoexcited carriers are generated as well the absorption or reflection of the probe pulse. Exciton effects should be less important for the exciton-phonon matrix elements. Jiang et al [131] recently studied exciton-photon and exciton-phonon matrix elements in SWNTs of arbitrary chirality and found that for RBM and $G$ modes, the exciton-phonon matrix elements with the Coulomb interaction turned on and in the free particle approximation are nearly identical. Hence we would not expect the initial phase of the oscillation to be strongly affected by the excitons. Comparing calculated exciton effects on the $\mathrm{CP}$ spectrum with experiment might prove very difficult since the effects of neighboring tubes and the local environment could be large.

Finally, as the experimental ability to make better samples (i.e. graphene nanoribbons, nanotubes of a fixed chirality) improves, we would expect that one would be able to generate coherent phonons in these samples that are not RBM, RBLM or $\mathrm{G}$ modes, but instead correspond to $q \neq 0$ acoustic modes. One could imagine ultrafast, pump-probe experiments where the pump and probe are spatially separated so that coherent acoustic phonons could be generated at one spatial point in the system but probed in a different point. The study of coherent phonons in carbon based nanostructures is only in its infancy and the future promises to be rewarding.

\section{Acknowledgments}

GDS and CJS are supported by the National Science Foundation under grant Nos. DMR-1105437 and OISE0968405, the Office of Naval Research under grant No. 
ONR-00075094. RS and KS acknowledge support from the MEXT grant, Japan (Nos. 20241203 and 23710118, respectively). JK acknowledges support from the National Science Foundation through Grant No. OISE-0968405, the Department of Energy BES Program through Grant No. DEFG02-06ER46308, and the Robert A Welch Foundation through Grant No. C-1509. We thank Avanash Rustagi for help with figure 34 .

\section{References}

[1] Aldersey-Williams H 1995 The Most Beautiful Molecule: The Discovery of the Buckyball (New York: Wiley)

[2] Prato M 1997 [60] Fullerene chemistry for materials science applications J. Mater. Chem. 7 1097-109

[3] Fowler P W and Manolopoulos D E 1995 An Atlas of Fullerenes (Oxford: Oxford University Press)

[4] Neto A H C, Guinea F, Peres N, Novoselov K and Geim A 2009 The electronic properties of graphene Rev. Mod. Phys. 81 109-62

[5] Novoselov K 2011 Nobel lecture: graphene: materials in the flatland Rev. Mod. Phys. 83837

[6] Geim A K 2011 Nobel lecture: random walk to graphene Rev. Mod. Phys. 83851

[7] Charlier J C, Blase X and Roche S 2007 Electronic and transport properties on nanotubes Rev. Mod. Phys. 79 677-732

[8] Harris P J F 2009 Carbon Nanotube Science: Synthesis Properties and Applications (Cambridge: Cambridge University Press)

[9] Anantram M P and Leonard F 2006 Physics of carbon nanotube electronic devices Rep. Prog. Phys. 69507

[10] Wong H S P and Akinwande D 2010 Carbon Nanotube and Graphene Device Physics (Cambridge: Cambridge University Press)

[11] Choi W and Lee J W 2011 Graphene: Synthesis and Applications vol 3 (Boca Raton, FL: CRC Press)

[12] Thomsen C, Strait J, Vardeny Z, Maris H J, Tauc J and Hauser J J 1984 Coherent phonon generation and detection by picosecond light pulses Phys. Rev. Lett. 53 989-92

[13] Cho G C, Kütt W and Kurz H 1990 Subpicosecond time-resolved coherent-phonon oscillations in GaAs Phys. Rev. Lett. 65 764-6

[14] Sun C K, Liang J C, Stanton C J, Abare A, Coldren L and DenBaars S P 1999 Large coherent acoustic-phonon oscillation observed in InGaN/GaN multiple-quantum wells Appl. Phys. Lett. 75 1249-51

[15] Sun C K, Liang J C and Yu X Y 2000 Coherent acoustic phonon oscillations in semiconductor multiple quantum wells with piezoelectric fields Phys. Rev. Lett. 84 179-82

[16] Yee K J, Lee K G, Oh E, Kim D S and Lim Y S 2002 Coherent optical phonon oscillations in bulk GaN excited by far below the band gap photons Phys. Rev. Lett. 88105501

[17] Stanton C J et al 2003 Coherent phonons, nanoseismology and $\mathrm{THz}$ radiation in $\mathrm{InGaN} / \mathrm{GaN}$ heterostructures Superlatt. Microstruct. 34525

[18] Chern G W, Sun C K, Sanders G D and Stanton C J 2004 Generation of coherent acoustic phonons in nitride-based semiconductor nanostructures Topics in Applied Physics vol 92, ed KT Tsen (New York: Springer) pp 339-94

[19] Wang J et al 2005 Propagating coherent acoustic phonon wave packets in $\operatorname{In}_{x} \mathrm{Mn}_{1-x} \mathrm{As} / \mathrm{GaSb}$ Phys. Rev. B 72153311

[20] Liu R et al 2005 Femtosecond pump-probe spectroscopy of propagating coherent acoustic phonons in $\operatorname{In}_{x} \mathrm{Ga}_{1-x} \mathrm{~N} / \mathrm{GaN}$ heterostructures Phys. Rev. B 72195335
[21] Sanders G D and Stanton C J 2006 Carrier dynamics and coherent acoustic phonons in nitride heterostructures Phys. Rev. B 74205303

[22] Noe G, Haugan H, Brown G, Sanders G, Stanton C and Kono J 2012 Coherent phonon dynamics in short-period InAs/GaSb superlattices Superlatt. Microstruct. 52 1071-7

[23] Ishioka K and Misochko O V 2010 Coherent Lattice Oscillations in Solids and Their Optical Control (Springer Series in Chemical Physics) vol 98 (Berlin: Springer) pp 23-46

[24] Hase M and Kitajima M 2010 Interaction of coherent phonons with defects and elementary excitations $J$. Phys.: Condens. Matter 22073201

[25] Dresselhaus M S, Dresselhaus G, Saito R and Jorio A 2005 Raman spectroscopy of carbon nanotubes Phys. Rep. 409 47-99

[26] Malard L, Pimenta M, Dresselhaus G and Dresselhaus M 2009 Raman spectroscopy in graphene Phys. Rep. 473 51-87

[27] Dresselhaus M S, Jorio A, Hofmann M, Dresselhaus G and Saito R 2010 Perspectives on carbon nanotubes and graphene Raman spectroscopy Nano Lett. 10 751-8

[28] Dresselhaus M, Jorio A and Saito R 2010 Characterizing graphene, graphite, and carbon nanotubes by Raman spectroscopy Annu. Rev. Condens. Matter Phys. 1 89-108

[29] Jorio A, Dresselhaus M S, Saito R and Dresselhaus G 2011 Raman Spectroscopy in Graphene Related Systems (Weinheim: Wiley-VCH)

[30] Iijima S 1991 Helical microtubules of graphitic carbon Nature 354 56-8

[31] Bethune D S et al 1993 Cobalt-catalysed growth of carbon nanotubes with single-atomic-layer walls Nature 363 605-7

[32] Iijima S and Ichihashi T 1993 Single-shell carbon nanotubes of 1-nm diameter Nature 363 603-5

[33] Joselevich E, Dai H, Liu J, Hata K and Windle A H 2008 Carbon nanotube synthesis and organization Carbon Nanotubes, Topics in Applied Physics (Berlin: Springer)

[34] Saito R, Dresselhaus G and Dresselhaus M S 1998 Physical Properties of Carbon Nanotubes (London: Imperial College Press)

[35] Saito R, Fujita M, Dresselhaus G and Dresselhaus M S 1992 Electronic structures of carbon fibers based on $\mathrm{C}_{60}$ Phys. Rev. B 46 1804-11

[36] Saito R, Dresselhaus G and Dresselhaus M S 2000 Trigonal warping effect of carbon nanotubes Phys. Rev. B 61 2981-90

[37] Jiang J et al 2007 Chirality dependence of exciton effects in single-wall carbon nanotubes: tight-binding model Phys. Rev. B 75035407

[38] Saito R, Takeya T, Kimura T, Dresselhaus G and Dresselhaus M S 1998 Raman intensity of single-wall carbon nanotubes Phys. Rev. B 57 4145-53

[39] Saito R, Hofmann M, Dresselhaus G, Jorio A and Dresselhaus M S 2011 Raman spectroscopy of graphene and carbon nanotubes Adv. Phys. 60 413-550

[40] Zhang B, Shimazaki K, Shiokawa T, Suzuki M, Ishibashi K and Saito R 2006 Stimulated Raman scattering from individual single-wall carbon nanotubes Appl. Phys. Lett. 88241101

[41] Kosynkin D V et al 2009 Longitudinal unzipping of carbon nanotubes to form graphene nanoribbons Nature. 458 872-6

[42] Han M Y, Özyilmaz B, Zhang Y and Kim P 2007 Energy band-gap engineering of graphene nanoribbons Phys. Rev. Lett. 98206805

[43] Masubuchi S, Ono M, Yoshida K, Hirakawa K and Machida T 2009 Fabrication of graphene nanoribbon by local anodic oxidation lithography using atomic force microscope Appl. Phys. Lett. 94082107 
[44] Tapaszto L, Dobrik G, Lambin P and Biro L P 2008 Tailoring the atomic structure of graphene nanoribbons by scanning tunnelling microscope lithography Nature Nanotechnol. 3 397-401

[45] Campos-Delgado J et al 2008 Bulk production of a new form of sp2 carbon: crystalline graphene nanoribbons Nano Lett. 8 2773-8

[46] Jia X, Campos-Delgado J, Terrones M, Meunier V and Dresselhaus M S 2011 Graphene edges: a review of their fabrication and characterization Nanoscale 3 86-95

[47] Sanders G D, Nugraha A R T, Saito R and Stanton C J 2012 Coherent radial-breathing-like phonons in graphene nanoribbons Phys. Rev. B 85205401

[48] Nanot S, Hároz E H, Kim J H, Hauge R H and Kono J 2012 Optoelectronic properties of single-wall carbon nanotubes Adv. Mater. 24 4977-94

[49] Makino K, Hirano A, Shiraki K, Maeda Y and Hase M 2009 Ultrafast vibrational motion of carbon nanotubes in different $\mathrm{pH}$ environments Phys. Rev. B 80245428

[50] Kim J H et al 2009 Chirality-selective excitation of coherent phonons in carbon nanotubes by femtosecond optical pulses Phys. Rev. Lett. 102037402

[51] Manzoni C, Gambetta A, Menna E, Meneghetti M, Lanzani G and Cerullo G 2005 Intersubband exciton relaxation dynamics in single-walled carbon nanotubes Phys. Rev. Lett. 94207401

[52] Lim Y S et al 2006 Coherent lattice vibrations in single-walled carbon nanotubes Nano Lett. 6 2696-700

[53] Lim Y S et al 2007 Chirality assignment of micelle-suspended single-walled carbon nanotubes using coherent phonon oscillations J. Korean Phys. Soc. $51306-11$

[54] Sanders G D et al 2009 Resonant coherent phonon spectroscopy of single-walled carbon nanotubes Phys. Rev B 79205434

[55] Lim Y S et al 2010 Resonant coherent phonon generation in single-walled carbon nanotubes through near-band-edge Excitation ACS Nano. 43222

[56] Booshehri L G et al 2011 Polarization dependence of coherent phonon generation and detection in highly-aligned single-walled carbon nanotubes Phys. Rev. B 83195411

[57] Gambetta A et al 2006 Real-time observation of nonlinear coherent phonon dynamics in single-walled carbon nanotubes Nature Phys. 2 515-20

[58] Lüer L, Gadermaier C, Crochet J, Hertel T, Brida D and Lanzani G 2009 Coherent phonon dynamics in semiconducting carbon nanotubes: a quantitative study of electron-phonon coupling Phys. Rev. Lett. 102127401

[59] Sasaki K and Saito R 2008 Pseudospin and deformation-induced gauge field in graphene Prog. Theor. Phys. Suppl. 176 253-78

[60] Bosma W B, Mukamel S, Greene B I and Schmitt-Rink S 1992 Femtosecond pump-probe spectroscopy of conjugated polymers: coherent and sequential contributions Phys. Rev. Lett. 68 2456-9

[61] Sanders G D, Sun C K, Fujimoto J G, Choi H K, Wang C A and Stanton C J 1994 Carrier-gain dynamics in $\mathrm{In}_{x} \mathrm{Ga}_{1-x} \mathrm{As} / \mathrm{Al}_{y} \mathrm{Ga}_{1-y}$ As strained-layer single-quantum-well diode lasers: comparison of theory and experiment Phys. Rev. B 50 8539-58

[62] Alexandrou A, Berger V and Hulin D 1995 Direct observation of electron relaxation in intrinsic GaAs using femtosecond pump-probe spectroscopy Phys. Rev. B 524654

[63] Choi C K et al 2001 Femtosecond pump-probe spectroscopy and time-resolved photoluminescence of an $\mathrm{In}_{x} \mathrm{Ga}_{1-x} \mathrm{~N} / \mathrm{GaN}$ double heterostructure Phys. Rev. B $\mathbf{6 3} 195302$
[64] Peng P, Milliron D J, Hughes S M, Johnson J C, Alivisatos A P and Saykally R J 2005 Femtosecond spectroscopy of carrier relaxation dynamics in type II $\mathrm{CdSe} / \mathrm{CdTe}$ tetrapod heteronanostructures Nano Lett. 5 1809-13

[65] Tribuzy C V B et al 2006 Femtosecond pump-probe spectroscopy of intersubband relaxation dynamics in narrow InGaAs/AlAsSb quantum well structures Appl. Phys. Lett. 89171104

[66] Gambetta A et al 2008 Sub-100 fs pump-probe spectroscopy of single wall carbon nanotubes with a $100 \mathrm{MHz}$ Er-fiber laser system Opt. Express 16 11727-34

[67] French P 1999 The generation of ultrashort laser pulses Rep. Prog. Phys. $\mathbf{5 8} 169$

[68] Krausz F and Ivanov M 2009 Attosecond physics Rev. Mod. Phys. 81163

[69] Henriksen N E and Engel V 2001 Femtosecond pump-probe spectroscopy: a theoretical analysis of transient signals and their relation to nuclear wave-packet motion Int. Rev. Phys. Chem. 20 93-126

[70] Kuznetsov A V and Stanton C J 1994 Theory of Coherent Phonon Oscillations in Semiconductors Phys. Rev. Lett. 73 3243-6

[71] Dumitrică T, Garcia M E, Jeschke H O and Yakobson B I 2004 Selective cap opening in carbon nanotubes driven by laser-induced coherent phonons Phys. Rev. Lett. 92117401

[72] Dumitrică T, Garcia M E, Jeschke H O and Yakobson B I 2006 Breathing coherent phonons and caps fragmentation in carbon nanotubes following ultrafast laser pulses Phys. Rev. B 74193406

[73] Ge Y, Yang F, Liang Q and Dong J 2011 Coherent phonons in excited-state carbon nanotubes: a simulation by tight-binding molecular dynamics Physica E 43 1585-91

[74] Nugraha A R T, Sanders G D, Sato K, Stanton C J, Dresselhaus M S and Saito R 2011 Chirality dependence of coherent phonon amplitudes in single-wall carbon nanotubes Phys. Rev. B 84174302

[75] Dresselhaus M, Dresselhaus G and Jorio A 2004 Unusual properties and structure of carbon nanotubes Annu. Rev. Mater. Res. 34 247-78

[76] Dresselhaus M S, Dresselhaus G and Ph A 2001 Carbon Nanotubes: Synthesis, Structure, Properties, and Applications (Berlin: Springer)

[77] Harris P J F 1999 Carbon Nanotubes and Related Structures: New Materials for the Twenty-First Century (Cambridge: Cambridge University Press)

[78] Terrones M 2003 Synthesis, properties, and applications of carbon nanotubes Annu. Rev. Mater. Sci. 33 419-501

[79] Saito R et al 2005 Cutting lines near the Fermi energy of single-wall carbon nanotubes Phys. Rev. B 72153413

[80] Blase X, Benedict L X, Shirley E L and Louie S G 1994 Hybridization effects and metallicity in small radius carbon nanotubes Phys. Rev. Lett. 72 1878-81

[81] Samsonidze G G et al 2004 Family behavior of the optical transition energies in single-wall carbon nanotubes of smaller diameters Appl. Phys. Lett. 85 5703-5

[82] Porezag D, Frauenheim T, Köhler T, Seifert G and Kaschner R 1995 Construction of tight-binding-like potentials on the basis of density-functional theory: application to carbon Phys. Rev. B 5112947

[83] Popov V N 2004 Curvature effects on the structural, electronic and optical properties of isolated single-walled carbon nanotubes within a symmetry-adapted non-orthogonal tight-binding model New J. Phys. 617

[84] Chuang S L 1995 Physics of Optoelectronic Devices (New York: Wiley)

[85] Bassani F and Parravicini G P 1975 Electronic States and Optical Transitions in Solids (New York: Pergamon) 
[86] Pedersen T G 2003 Analytic calculation of the optical properties of graphite Phys. Rev. B 67113106

[87] Taft E A and Philipp H R 1965 Optical properties of graphite Phys. Rev. 138 A197-202

[88] Ehrenreich H and Cohen M H 1959 Self-consistent field approach to the many-electron problem Phys. Rev. $115786-90$

[89] Yang L, Deslippe J, Park C H, Cohen M L and Louie S G 2009 Excitonic effects on the optical response of graphene and bilayer graphene Phys. Rev. Lett. 103186802

[90] Spataru C D, Ismail-Beigi S, Benedict L X and Louie S G 2004 Excitonic effects and optical spectra of single-walled carbon nanotubes Phys. Rev. Lett. 92077402

[91] Ando T 1997 Excitons in carbon nanotubes J. Phys. Soc. Japan 66 1066-73

[92] Dresselhaus M S, Dresselhaus G, Saito R and Jorio A 2007 Exciton photophysics of carbon nanotubes Annu. Rev. Phys. Chem. 58 719-47

[93] Kahn D, Kim K W and Stroscio M A 2001 Quantized vibrational modes of nanospheres and nanotubes in the elastic continuum model J. Appl. Phys. 89 5107-11

[94] Mahan G 2002 Oscillations of a thin hollow cylinder: carbon nanotubes Phys. Rev. B 65235402

[95] Raichura A, Dutta M and Stroscio M A 2003 Quantized acoustic vibrations of single-wall carbon nanotube J. Appl. Phys. 94 4060-5

[96] Raichura A, Dutta M and Stroscio M A 2004 Quantized optical vibrational modes of finite-length multi wall nanotubes: optical deformation potential Superlatt. Microstruct. 35 147-53

[97] Raichura A, Dutta M and Stroscio M A 2004 Continuum model for acoustic phonons in nanotubes: phonon bottleneck Phys. Status Solidi b 241 3448-53

[98] Chico L, Pérez-Álvarez R and Cabrillo C 2006 Low-frequency phonons in carbon nanotubes: a continuum approach Phys. Rev. B 73075425

[99] Qian J, Allen M J, Yang Y, Dutta M and Stroscio M A 2009 Quantized long-wavelength optical phonon modes in graphene nanoribbon in the elastic continuum model Superlatt. Microstruct. 46 881-8

[100] Jishi R A, Venkataraman L, Dresselhaus M S and Dresselhaus G 1993 Phonon modes in carbon nanotubules Chem. Phys. Lett. 20977

[101] Lobo C and Martins J L 1997 Valence force field model for graphene and fullerenes Z. Phys. D. 39 159-64

[102] Mahan G D and Jeon G S 2004 Flexure modes in carbon nanotubes Phys. Rev. B 70075405

[103] Mazzamuto F, Saint-Martin J, Valentin A, Chassat C and Dollfus P 2011 Edge shape effect on vibrational modes in graphene nanoribbons: a numerical study J. Appl. Phys. 109064516

[104] Jeon G S and Mahan G 2005 Theory of infrared-active phonons in carbon nanotubes Phys. Rev. B 72155415

[105] Kürti J, Kresse G and Kuzmany H 1998 First-principles calculations of the radial breathing mode of single-wall carbon nanotubes Phys. Rev. B 58 8869-72

[106] Sánchez-Portal D, Artacho E, Soler J M, Rubio A and Ordejón P 1999 Ab initio structural, elastic, and vibrational properties of carbon nanotubes Phys. Rev. B 5912678

[107] Kürti J, Zólyomi V, Kertesz M and Sun G 2003 The geometry and the radial breathing mode of carbon nanotubes: beyond the ideal behaviour New J. Phys. 5125

[108] Dubay O and Kresse G 2003 Accurate density functional calculations for the phonon dispersion relations of graphite layer and carbon nanotubes Phys. Rev. B 67035401

[109] Ye L H, Liu B G, Wang D S and Han R 2004 Ab initio phonon dispersions of single-wall carbon nanotubes Phys. Rev. B 69235409
[110] Gillen R, Mohr M, Thomsen C and Maultzsch J 2009 Vibrational properties of graphene nanoribbons by first-principles calculations Phys. Rev. B 80155418

[111] Madelung O 1978 Introduction to Solid-State Theory (Berlin: Springer)

[112] Jiang J W, Tang H, Wang B S and Su Z B 2006 Chiral symmetry analysis and rigid rotational invariance for the lattice dynamics of single-wall carbon nanotubes Phys. Rev. B 73235434

[113] Jiang J et al 2005 Electron-phonon matrix elements in single-wall carbon nanotubes Phys. Rev. B 72235408

[114] Grüneis A 2004 Resonance Raman Spectroscopy of Single Wall Carbon Nanotubes (Sendai: Tohoku University)

[115] Sasaki K, Saito R, Dresselhaus G, Dresselhaus M S, Farhat $\mathrm{H}$ and Kong J 2008 Chirality-dependent frequency shift of radial breathing mode in metallic carbon nanotubes Phys. Rev. B 78235405

[116] Sanders G D et al 2011 Coherent phonons in carbon nanotubes and graphene AIP Conf. Proc. 141631

[117] Machón M, Reich S, Telg H, Maultzsch J, Ordejón P and Thomsen C 2005 Strength of radial breathing mode in single-walled carbon nanotubes Phys. Rev. B 71035416

[118] Kato K, Ishioka K, Kitajima M, Tang J, Saito R and Petek H 2008 Coherent phonon anisotropy in aligned single-walled carbon nanotubes Nano Lett. 8 3102-8

[119] Ando T 2005 Theory of electronic states and transport in carbon nanotubes J. Phys. Soc. Japan 74 777-817

[120] Capaz R B, Spataru C D, Ismail-Beigi S and Louie S G 2006 Diameter and chirality dependence of exciton properties in carbon nanotubes Phys. Rev. B 74121401

[121] Dukovic G, Wang F, Song D, Sfeir M Y, Heinz T F and Brus L E 2005 Structural dependence of excitonic optical transitions and band-gap energies in carbon nanotubes Nano Lett. 5 2314-8

[122] Miyauchi Y et al 2007 Dependence of exciton transition energy of single-walled carbon nanotubes on surrounding dielectric materials Chem. Phys. Lett. 442 394-9

[123] Kim J H et al 2009 Polarization anisotropy of transient carrier and phonon dynamics in carbon nanotubes $J$. Appl. Phys. 105103506

[124] Pint C L et al 2010 Dry contact transfer printing of aligned carbon nanotube patterns and characterization of their optical properties for diameter distribution and alignment ACS Nano 4 1131-45

[125] Son Y W, Cohen M L and Louie S G 2006 Energy gaps in graphene nanoribbons Phys. Rev. Lett. 97216803

[126] Raza H and Kan E C 2008 Armchair graphene nanoribbons: electronic structure and electric-field modulation Phys. Rev. B 77245434

[127] Fujita M, Wakabayashi K, Nakada K and Kusakabe K 1996 Peculiar localized state at zigzag graphite edge J. Phys. Soc. Japan 65 1920-3

[128] Nakada K, Fujita M, Dresselhaus G and Dresselhaus M S 1996 Edge state in graphene ribbons: nanometer size effect and edge shape dependence Phys. Rev. B 54 17954-61

[129] Pisani L, Chan J A, Montanari B and Harrison N M 2007 Electronic structure and magnetic properties of graphitic ribbons Phys. Rev. B 75064418

[130] Sasaki K, Murakami S and Saito R 2006 Gauge field for edge state in graphene J. Phys. Soc. Japan 75074713

[131] Jiang J et al 2007 Exciton-photon, exciton-phonon matrix elements, and resonant Raman intensity of single-wall carbon nanotubes Phys. Rev. B $\mathbf{7 5} 035405$ 